\title{
Treewidth : structural properties and algorithmic insights
}

Citation for published version (APA):

Marchal, L. (2012). Treewidth : structural properties and algorithmic insights. [Doctoral Thesis, Maastricht University]. Datawyse / Universitaire Pers Maastricht. https://doi.org/10.26481/dis.20120405lm

Document status and date:

Published: 01/01/2012

DOI:

10.26481/dis.20120405Im

Document Version:

Publisher's PDF, also known as Version of record

\section{Please check the document version of this publication:}

- A submitted manuscript is the version of the article upon submission and before peer-review. There can be important differences between the submitted version and the official published version of record.

People interested in the research are advised to contact the author for the final version of the publication, or visit the DOI to the publisher's website.

- The final author version and the galley proof are versions of the publication after peer review.

- The final published version features the final layout of the paper including the volume, issue and page numbers.

Link to publication

\footnotetext{
General rights rights.

- You may freely distribute the URL identifying the publication in the public portal. please follow below link for the End User Agreement:

www.umlib.nl/taverne-license

Take down policy

If you believe that this document breaches copyright please contact us at:

repository@maastrichtuniversity.nl

providing details and we will investigate your claim.
}

Copyright and moral rights for the publications made accessible in the public portal are retained by the authors and/or other copyright owners and it is a condition of accessing publications that users recognise and abide by the legal requirements associated with these

- Users may download and print one copy of any publication from the public portal for the purpose of private study or research.

- You may not further distribute the material or use it for any profit-making activity or commercial gain

If the publication is distributed under the terms of Article $25 \mathrm{fa}$ of the Dutch Copyright Act, indicated by the "Taverne" license above, 


\section{Treewidth \\ structural properties and algorithmic insights}

Lambertus Marchal 
This book was typeset by the author using LaTeX.

All rights reserved. No part of this publication may be reproduced, stored in a retrieval system, or transmitted, in any form, or by any means, electronic, mechanical, photocopying, recording or otherwise, without the prior permission in writing from the author.

Treewidth: structural properties and algorithmic insights

(C) Lambertus Marchal, 2012

Published by Universitaire Pers Maastricht

ISBN 9789461591340

Printed in the Netherlands by Datawyse 


\title{
Treewidth \\ structural properties and algorithmic insights
}

\author{
Proefschrift
}

ter verkrijging van de graad van doctor aan de Universiteit Maastricht, op gezag van Rector Magnificus,

Prof. mr. G.P.M.F. Mols, volgens het besluit van het College van Decanen,

in het openbaar te verdedigen

op donderdag 5 april 2012 om 14.00 uur

door

Lambertus Marchal

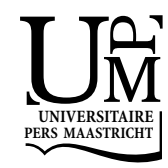




\section{Promotor:}

Prof. dr. ir. C.P.M. van Hoesel

\section{Copromotor:}

Dr. A. Grigoriev

\section{Beoordelingscommissie:}

Prof. dr. R.J. Müller (voorzitter)

Dr. A. Berger

Dr. H.L. Bodlaender (Universiteit Utrecht)

Prof. dr. ir. A.M.C.A. Koster (RWTH Aachen University)

Dit onderzoek werd financieel mogelijk gemaakt door Maastricht Research School of Economics of Technology and Organizations (METEOR). 


\section{Acknowledgements}

This dissertation would not have been realized without the help of several individuals who in one way or another extended their valuable assistance and contributed to its creation and completion. I would like to take the opportunity to acknowledge some of them here.

First, I want to express my gratitude to my promotor, Stan van Hoesel. On an enjoyable spring day in 2005, he succeeded to kindle my interest in a challenging topic, the full scope of which is still beyond my mind to grasp. Throughout the years of my doctoral studies he provided his expertise, while allowing me the room to work in my own way. His patience and reassuring composure have benefited me greatly.

Furthermore, I am heartily thankful to my copromotor, Alexander Grigoriev, for the many sessions that got things going again when I got stuck at writing. His creativity has been an inexhaustible source of new research directions to explore. Writing a dissertation can at times be a daunting and frustrating process. His ability to put things into perspective has always been most welcome. One could not wish for a more enthusiastic supervisor.

Most of the research that led to this dissertation has been conducted in close collaboration with several co-authors. I am indebted to Hans Ensinck and Arie Koster for their valuable contributions to respectively Chapter 3 and 4 of this dissertation.

I would like to thank my colleagues for their useful suggestions and for creating a pleasant work atmosphere in Maastricht. I thank my friends and family for providing me with the necessary distractions from writing.

Finally, my biggest thanks go to Natalya. She has been much more than the co-author of Chapters 5 and 6 . Her loving support, encouragement and leading example have been the inspiration to complete this work. I feel very privileged to have her at my side.

Bert Marchal

's-Gravenhage, February 2012 



\section{Contents}

1 Introduction 11

1.1 Graph theory . . . . . . . . . . . . . . . . . . . . 12

1.2 Algorithms and complexity . . . . . . . . . . . . . . . . . . 14

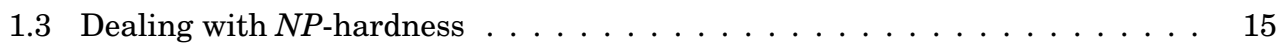

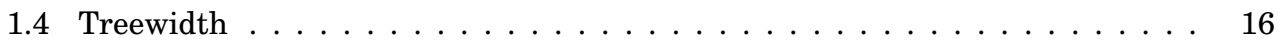

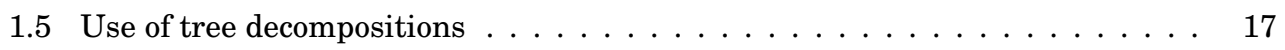

1.5.1 when treewidth is not bounded . . . . . . . . . . . . . 19

1.6 Dynamic programming on tree decompositions . . . . . . . . . . . . . . 20

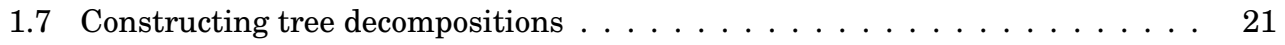

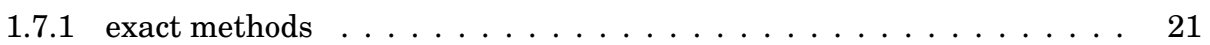

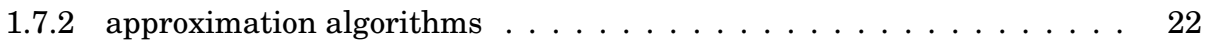

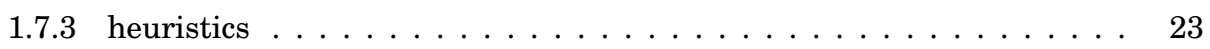

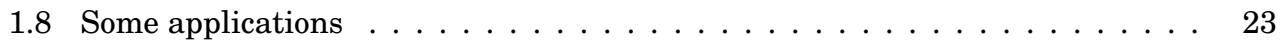

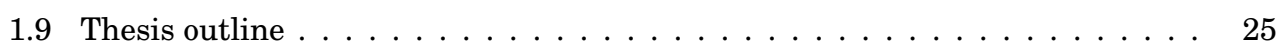

1.10 Published material . . . . . . . . . . . . . . . . 26

2 Preliminaries 27

2.1 Graph terminology . . . . . . . . . . . . . . . . . . 27

2.2 Planar graphs . . . . . . . . . . . . . . . . . . . . . . . . . . . . . . . . . . . .

2.3 Graph minors . . . . . . . . . . . . . . . . . . 30

2.4 Treewidth . . . . . . . . . . . . . . . . . . . . . . . . . . . . . .

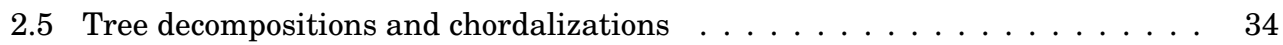

3 Branch and Bound $\quad 37$

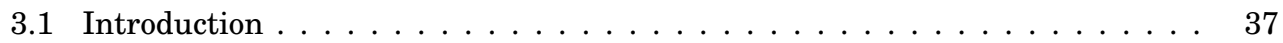

3.2 Preliminaries . . . . . . . . . . . . . . . . . . 39

3.3 the Necklace structure $\ldots \ldots \ldots \ldots \ldots \ldots \ldots \ldots$ 


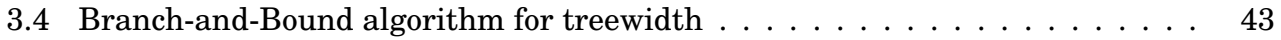

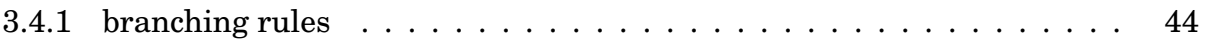

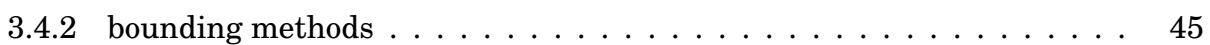

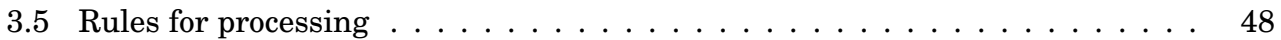

3.5 .1 simplicial vertices $\ldots \ldots \ldots \ldots \ldots \ldots$

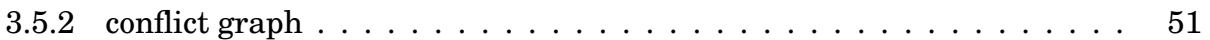

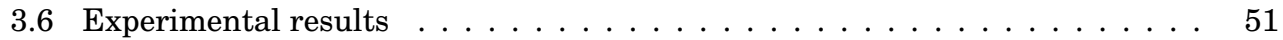

3.6 .1 Grid graphs . . . . . . . . . . . . . . . . . 52

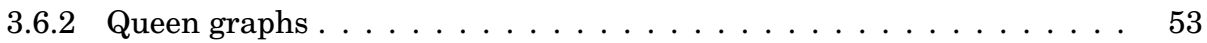

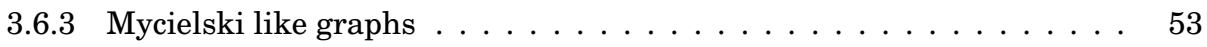

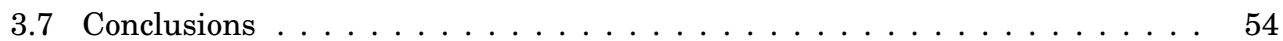

4 Local Search $\quad 57$

4.1 Preliminaries . . . . . . . . . . . . . . . . . . 58

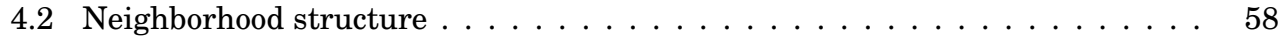

4.2 .1 procedure 1 : removing a fill-in pair . . . . . . . . . . . . 60

4.2 .2 procedure 2 : regaining minimality . . . . . . . . . . 63

4.3 Local search . . . . . . . . . . . . . . . . . . . . . . . . . . 68

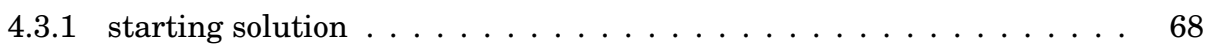

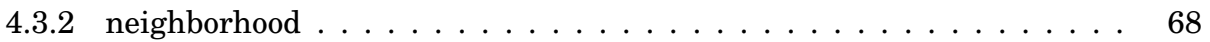

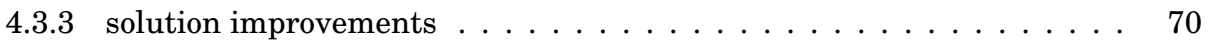

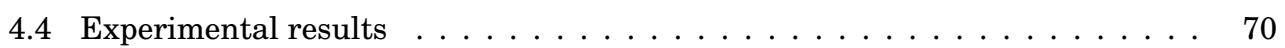

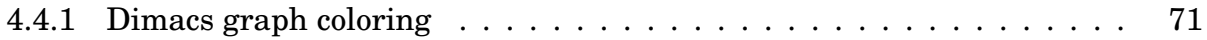

4.4 .2 frequency assignment . . . . . . . . . . . . . . . 71

4.4 .3 Bayesian networks $\ldots \ldots \ldots \ldots \ldots \ldots$

5 Grid Minors $\quad 75$

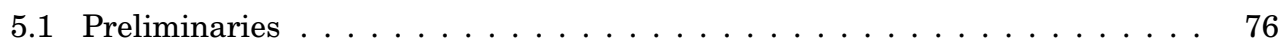

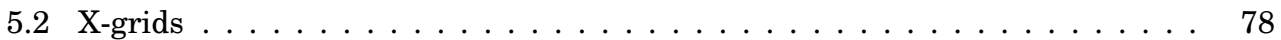

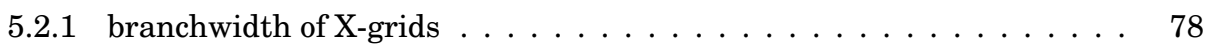

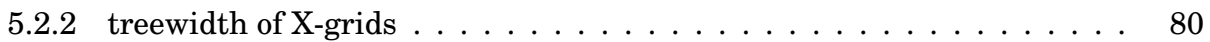

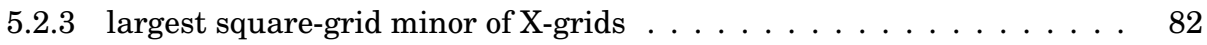

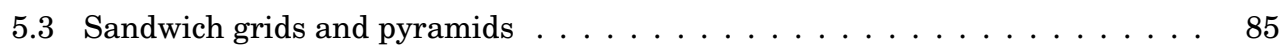

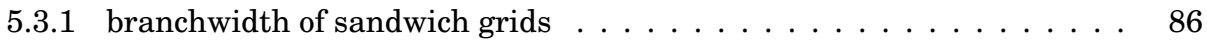

5.3 .2 treewidth of sandwich grids and pyramids . . . . . . . . . . . 87

5.3.3 square-grid minor of sandwich grids and pyramids . . . . . . . . . . . . 87

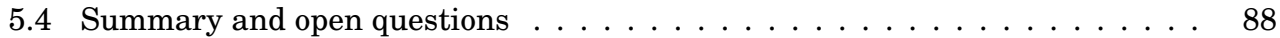


$6 H$-Subgraph Edge Deletion $\quad 91$

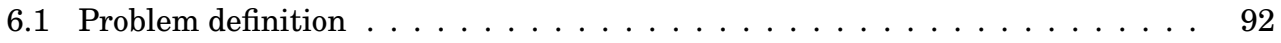

6.2 MSOL Formulations . . . . . . . . . . . . . . . . . . . . . . 93

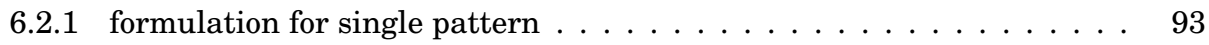

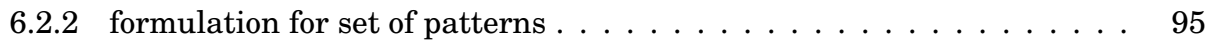

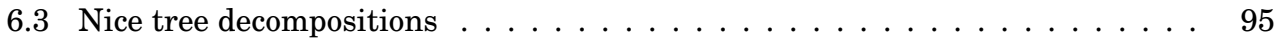

6.4 DP for text graphs with bounded degree . . . . . . . . . . . . . . . 96

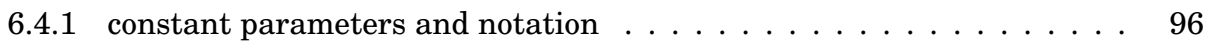

6.4 .2 dynamic program and results . . . . . . . . . . . . . 97

6.5 Dynamic program for clique patterns . . . . . . . . . . . . . . . 100

6.5 .1 dynamic program and results . . . . . . . . . . . . . . . . . . . . . . . . . . . . . 100

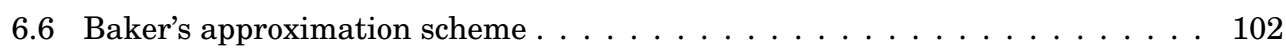

6.6 .1 bounded outerplanarity index . . . . . . . . . . . . . . . . 102

6.6 .2 approximation schemes . . . . . . . . . . . . . . 103

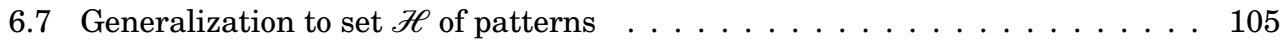

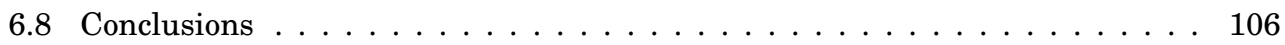

$\begin{array}{ll}\text { Bibliography } & 107\end{array}$

$\begin{array}{ll}\text { Nederlandse Samenvatting } & 115\end{array}$

$\begin{array}{lr}\text { Curriculum Vitae } & 119\end{array}$ 



\section{Chapter 1}

\section{Introduction}

Graphs are structures that are used to model a broad spectrum of problems that arise from real-world situations. These problems can originate from a variety of applications such as data analysis, electrical circuit design, logistics, scheduling, social networks, telecommunication and many others. To solve such a problem using a graph theoretic approach, one constructs a graph that represents the real-world setting of the problem in a mathematical way. Once this is done, the problem is stated in graph theoretical terms and an algorithm taking the graph as an input is used to generate a solution to the problem. Finally, the mathematical solution obtained this way must be translated back into the real-world context.

The running time of an algorithm depends heavily on the difficulty of the problem itself, but also on the complexity of the graph that models the framework of the problem. Some problems are easy in the sense that there is an algorithm that can solve them in an efficient way, independent of the structure of the input graph. In this context, efficiency of the algorithm usually means that the running time of the algorithm only grows polynomially in the size of the input graph. However, a lot of practically relevant problems are hard, meaning that the running time of algorithms solving them grows at least exponentially in the size of the input graph.

When dealing with hard problems, one might settle for a close-to-optimal solution that can be obtained in polynomial time. One can also try to exploit the structure of the input graph. It turns out that a lot of problems that are hard on general input graphs, can still be solved efficiently on input graphs that possess some special structural property. Based on properties as 'drawable in the plane without edges crossing' or 'all vertices having $k$ neighbors', graphs are categorized in a vast number of classes. One graph class in particular that facilitates many otherwise hard problems, is the class of trees. Trees are connected graphs that do not contain cycles. The ease with which some hard problems can be solved on trees, often carries over to input graphs that are in some sense 'treelike'. A parameter 
that measures the similarity of a graph to a tree is the treewidth of the graph. The lower its treewidth, the more the graph resembles a tree and the faster many problems can be solved on this particular graph.

Algorithms that exploit the treelike property of graphs often take as input a so called tree decomposition of the graph rather than the graph itself. A graph has usually numerous tree decompositions, each having their own width. The lower the width of a tree decomposition, the faster the algorithm runs on this tree decomposition. The ability to construct tree decompositions that have low width is therefore highly valuable when dealing with hard problems. The treewidth of the graph equals the lowest width over all tree decompositions of a graph. Unfortunately, constructing a tree decomposition of lowest possible width (and thus determining the treewidth of the graph) is a hard problem in itself for general graphs. This thesis deals with various aspects of treewidth and tree decompositions.

In the remainder of this chapter we provide some background information on the theory of graphs and algorithms on graphs. In particular we focus on the role that treewidth and tree decompositions play in many of such algorithms. The basic terminology that will be utilized throughout the thesis is clarified in Chapter 2. In Chapter 3, we present an exact algorithm that determines the treewidth of a graph. A heuristic for bounding the treewidth from above is introduced in Chapter 4. The relationship between treewidth of planar graphs and some other parameters of planar graphs will be examined in Chapter 5. To conclude the thesis, various algorithms are presented in Chapter 6 that illustrate how tree decompositions can be exploited to solve a particular graph problem.

\subsection{Graph theory}

A graph is a mathematical object that captures the notion of connectivity between a set of objects. The objects are represented by the vertices of the graph and a pairwise relation between two objects is represented by an edge between the vertices that correspond to the objects. In mathematics and computer science, graph theory is the study of graphs.

Some say that graph theory originated from a puzzle that was once posed by the townsfolk of Königsberg, Prussia in the early 1700's, see e.g. (16). Königsberg was built largely on an island in the Pregel river. The island is located near where two branches of the river join, and the borders of the town spread over to the banks of the rivers. Between the four land masses that the town occupied, seven bridges had been erected, as is illustrated in the leftmost picture in Figure 1.1.

The people of Königsberg supposedly posed the following question: Is it possible to take a walk through town, crossing each of the seven bridges just once? In 1736, Swiss mathematician Leonhard Euler, having heard of the problem, used the graph in the rightmost picture from Figure 1.1 to show that such a walk could not be made. The four land masses 

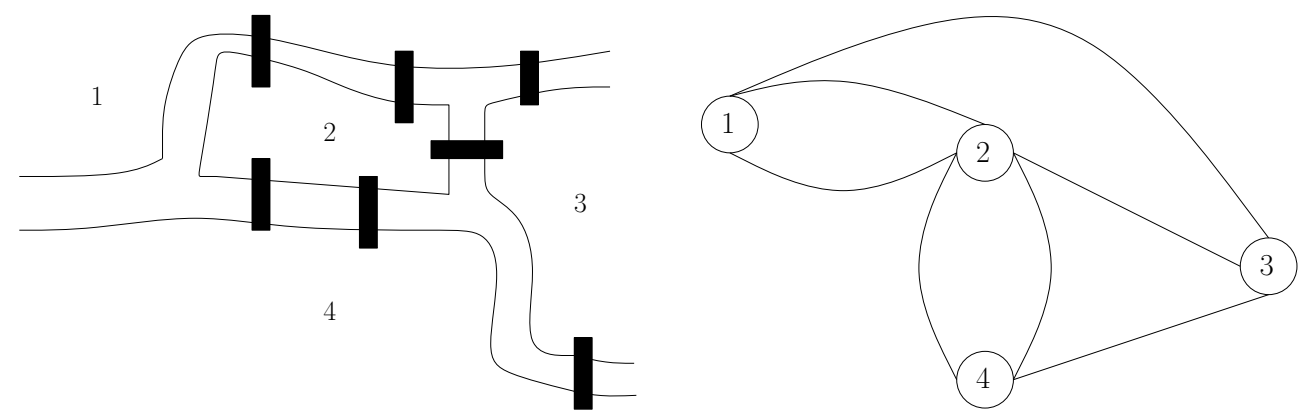

Figure 1.1: the seven bridges of Königsberg and a graph representing the setting

are represented by vertices in the graph and every bridge between two land masses is represented by an edge between the two vertices that correspond to the land masses. The degree of a vertex in a graph is the number of edges emanating from the vertex. Euler's argument was quite simple: suppose one could make such a walk, then there are at least two land masses that neither form the start nor the beginning of the walk. Whenever such a land mass is entered during the walk, it has to be left again to finish the walk. Hence there must be at least two vertices in the graph that have even degree, which is not the case.

Graphs have since then become an essential means for transforming real-life settings into abstract mathematical models. Problems that arise from such real-world settings are often decision problems or optimization problems. A decision problem is a question that can be answered by either "yes" or "no", depending on the input parameters. An optimization problem is a problem of optimizing (either maximizing or minimizing) some value based on the input parameters, for example to find the shortest route between two cities on a road map. Optimization problems can be cast as decision problems, e.g., instead of asking for the length of the shortest route between two cities one can ask whether between the two cities a route exists of length at most $k$, for some integer $k$.

For this specific problem, the real-world setting would be the network of roads between the two cities. A graph theoretical representation of this setting could be a graph in which the vertices represent all intersections and ends of the roads while the edges represent the road segments between all intersections and ends of the roads. Simple models like this normally allow for many extensions to make them more conform to the real-world setting. One might for example assign a weight to each edge that denotes the length of the corresponding road segment. For more information on graph theory and its applications, we refer to (57) and (68). 


\subsection{Algorithms and complexity}

Apart from their purpose, algorithms can differ in running time and memory space they require to fabricate an answer. The demand for these resources is usually expressed in the input size of the problem, i.e., the number of bits needed to denote the input. For problems on graphs, the input size of the problem is usually the number of vertices in the graph. In computer science, an algorithm is called a polynomial-time algorithm if the time needed to run it grows only polynomially in the size of the input. If its running time grows exponentially in the input size, the algorithm is called an exponential-time algorithm. Polynomial-space and exponential-space algorithms can be defined in a similar manner. Very roughly speaking, polynomial algorithms are fast and efficient while exponential algorithms are slow or require too much memory to be useful in practice. For an overview of some algorithms on graphs, we refer to (107).

Based on the time and space requirements of algorithms to solve them, problems on graphs are clustered into a number of complexity classes. At the moment of writing this thesis, there are nearly 500 complexity classes (see (1) for an exhaustive overview) of which we will mention only a few here. One of the most fundamental complexity classes is the class $P$. It contains all decision problems that can be solved in polynomial time. As a rule of thumb, we say that $P$ is the class of computational problems which are 'efficiently solvable' or 'tractable'. A superset of $P$ is the class $N P$ of so called "nondeterministic polynomialtime" problems. $N P$ is defined as the set of decision problems for which a possible solution can be verified in polynomial time. Class $P$ is a subset of $N P$, but there are a lot of graph problems in $N P$ for which no polynomial-time algorithms are known. For this reason it is widely believed that $P$ is a strict subset of $N P$.

A problem in $N P$ is said to be $N P$-complete if a polynomial-time algorithm for this problem would imply the existence of polynomial-time algorithms for all other problems in $N P$. This is why $N P$-complete problems are considered to be the hardest problems in NP. For more details on the theory of $N P$-completeness, we refer to (63).

A problem is called $N P$-hard if it is as least as hard as any other problem in the class $N P$. More formally, a problem $H$ is $N P$-hard if and only if there is an $N P$-complete problem $L$ that is in polynomial-time reducible to $H$. This basically means that if some oracle would give us a solution to $H$, then we can solve $L$ (and thereby any problem in the class $N P$ ) in polynomial time. We refer to (76) for an elaborate list of practically relevant $N P$-hard optimization problems.

Figure 1.2 displays the relation between the classes $P, N P, N P$-complete and $N P$-hard for the case where $P \neq N P$ versus the case where $P=N P$. One of the central topics in this thesis is the determination of the treewidth of a graph. The decision version of this problem, i.e. "given a graph $G$ and an integer $k$, decide whether the treewidth of $G$ is at most $k$ ", was 


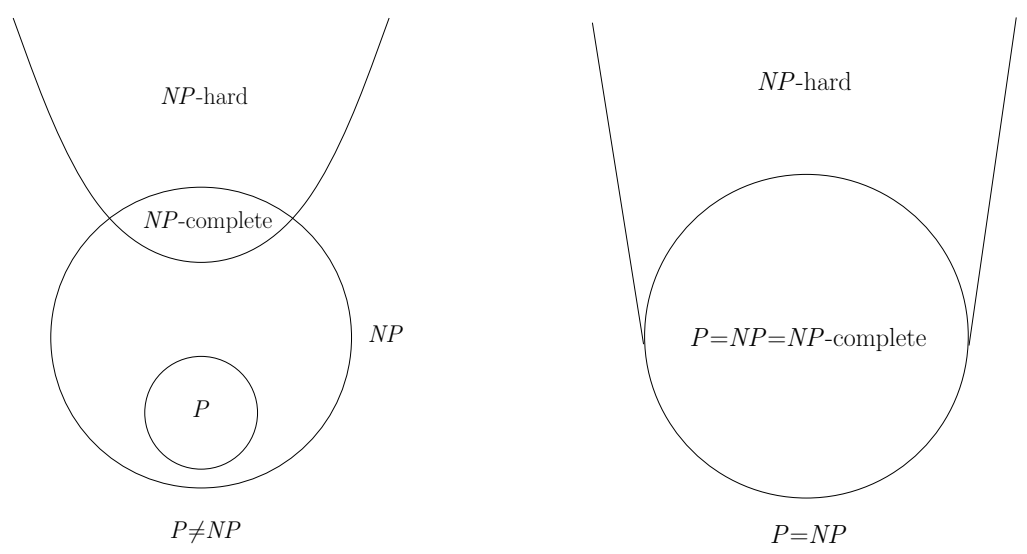

Figure 1.2: Euler diagram for problems in $P, N P, N P$-complete and $N P$-hard

shown to be $N P$-complete in (7). This implies that treewidth determination is an NP-hard problem.

Parameterized complexity is a branch of computational complexity theory that focuses on classifying problems according to their difficulty with respect to multiple input parameters. While in the classical setting the complexity of a problem is measured as a function in the number of bits of input, in parameterized complexity, it is measured as a function in two or more input parameters.

A number of problems that are $N P$-hard in the classical setting, are so called fixed parameter tractable in parameterized complexity. We speak of fixed parameter tractability of a decision problem if the function measuring its complexity is exponential in only one (small) input parameter $k$ and polynomial in the input size of the problem. By fixing the value of the input parameter $k$, we say that the problem is parameterized in $k$. While doing so, the exponential factor disappears from the computational complexity of the problem, making the problem tractable. The class of fixed parameter tractable problems is denoted by FPT.

A major incentive for investigating the treewidth problem is that a lot of NP-hard problems on graphs are in the class FPT, where the treewidth of the graph plays the role of the fixed parameter, i.e., these problems are tractable when they are parameterized by treewidth.

\subsection{Dealing with $N P$-hardness}

In the previous section we indicated that determination of treewidth is an NP-hard problem. Various ways of dealing with $N P$-hard problems have been suggested over the course of time. The most uncomplicated is by brute force, i.e., just enumerate and evaluate all possible 
solutions and pick the best one. The amount of time needed limits the usability of this approach to only small graph instances. Methods like branch-and-bound are based on the same principle of enumerating all solution candidates. However, they do this in a systematic way in which subsets of fruitless candidates are discarded in massive numbers. Although they are able to handle $N P$-hard problems on larger instances than the brute force method, their running times are still at least exponential. Indeed, assuming that $P \neq N P$, there are simply no polynomial-time algorithms that return exact solutions to $N P$-hard problems.

A more practical way to cope with $N P$-hard problems is therefore using approximation algorithms or heuristics. Approximation algorithms are efficient and guarantee that the returned solution is at most a factor (either fixed or depending on some input parameter) away from optimal. Heuristics are quick and the solution they return is usually reasonably good, but can in the worst case be arbitrarily far away from optimal. Their performance typically relies on educated guesses and common sense rather than on mathematically wellfounded methods.

A third approach to $N P$-hard problems is by taking advantage of certain properties of the input graph. It is well known that many problems that are $N P$-hard in general become efficiently solvable when they are restricted to input graphs that belong to a certain graph class. For an exhaustive list of graph classes, the complexity of recognizing these classes and a lot of additional information, we refer to $(45 ; 66)$.

One property that has proven to be particularly useful in this context is for the input graph to be a tree. Most real-world scenarios can only be modeled by graphs that are more complex than trees. This is where treewidth comes into play. The treewidth of a graph is a measure for how close the graph resembles a tree. The lower the treewidth, the more similar the graph is to a tree. NP-hard problems that can be efficiently solved on trees are also easily solvable on graphs that have low treewidth, because there are so called FPT-algorithms (Fixed Parameter Tractable) that solve these problems in time that is only exponential in the treewidth of the graph.

\subsection{Treewidth}

The notion of treewidth that will be used throughout this thesis was introduced by Robertson and Seymour in a series of papers on graph minor theory, see (95). Independently, Arnborg and Proskurowski introduced the equivalent notion of partial $k$-trees, i.e., a graph has treewidth at most $k$ if and only if it is a partial $k$-tree (8).

There is little doubt that treewidth is one of the most important concepts that was introduced in the field of mathematics and computer science during the last decades. Ever since their introduction, treewidth and closely related notions like pathwidth and branchwidth have been the subject of study by numerous scientists in the field. The hardness of deter- 
mining the treewidth of a graph makes the topic challenging from a theoretical viewpoint. From a more practical angle, the study of treewidth attracted a lot of interest because it can serve as a mathematical tool in dealing with other $N P$-hard combinatorial problems.

The definition of treewidth invented by Robertson and Seymour is based on the notion of tree decomposition. A tree decomposition of a graph $G$ is a way to represent $G$ as a tree. It consists of a tree $T$ and a set of subsets of vertices of $G$. Each subset forms a node of $T$ and is usually referred to as a 'bag'. The tree $T$ and its bags form a valid tree decomposition of graph $G$, if the following three conditions hold:

1. Each vertex of $G$ is present in at least one bag of $T$.

2. For each edge of $G$, there is at least one bag in $T$ that contains both end vertices of the edge.

3. For each vertex $v$ of $G$, the bags of $T$ that contain $v$ form a connected subtree of $T$.

The width of a tree decomposition is equal to the number of vertices in its largest bag minus one. The treewidth of a graph is defined to be the minimum width over all tree decompositions of the graph.

Another way to describe treewidth utilizes the notion of chordalization. A chordalization of a graph can be obtained by adding edges to the graph (if necessary) until for all cycles in the graph there is an edge connecting two vertices that are non-adjacent on the cycle. A subset of vertices in a graph that are pairwise connected by an edge is called a clique. The width of a chordalization is equal to the size of the largest clique in the chordalization minus one. The treewidth of a graph equals the minimum width over all its chordalizations. Formal definitions of tree decomposition and chordalization will be introduced in Chapter 2 .

Trees contain no cycles, so they form their own chordalization and having a largest clique of size two (omitting graphs on a single vertex), the treewidth of a tree equals one. Cliques are also chordalizations of themselves and hence the treewidth of a clique on $n$ vertices is equal to $n-1$. As another example, the infamous Petersen graph is shown as the leftmost picture in Figure 1.3. For the Petersen graph, it is known that the treewidth equals 4 . The tree decomposition and chordalization of the Petersen graph that are also depicted in Figure 1.3 both have a width of 4 and hence they are optimal.

\subsection{Use of tree decompositions}

In general, solving a problem becomes easier (faster in terms of running time) when the graph instance gets smaller. Based on this idea, hard problems can often be solved by a so called "divide-and-conquer" strategy. Given a problem on a graph, the idea is to recursively 

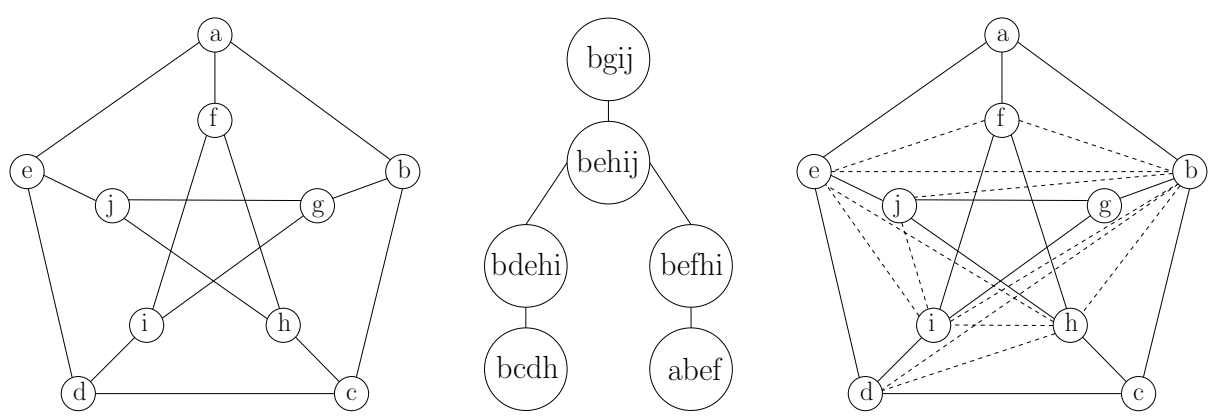

Figure 1.3: From left to right are depicted the Petersen graph, a tree decomposition of the Petersen graph and a chordalization of the Petersen graph.

split up the input graph into a number of smaller graphs. On each of these small graphs a problem of the same nature is defined that can be solved quickly.

To obtain the solution for the original problem, the solutions on the small graphs have to be joined together in a consistent way. However, the quality of a decision that is made in one small part of the input graph will very likely depend on the decisions that were made in other parts of the graph. The effect of a decision made in one part of the graph propagates to another part via all paths in the graph connecting the two parts. The more such paths there are, the more ways there are to combine solutions from the two parts. This makes it hard to find a solution for the combined parts that is feasible. Combining solutions to the two separate parts into an optimal solution for the combined parts is even harder.

In dealing with how to combine the partial solutions the property of the input graph having low or in some sense 'bounded' treewidth turns out to be algorithmically helpful. Getting some insight as to why this is the case can best be realized by means of simple trees. By absence of any cycles, two parts of a tree are connected to each other via just one single path. Hence, the influence of a local decision made in one part of the tree to a decision in another part of the tree can be captured by information concerning only the unique path between the two parts. To some extent, this easiness carries over to graphs that have low treewidth.

Consider a graph $G$ on which some problem is defined. Assume we are given a tree decomposition of $G$ of width $k$, where $k$ is a small constant. By deleting an internal bag from the tree decomposition we split up the tree into at least two subtrees. Consider the subgraphs of $G$ that are induced by the vertices in these subtrees. They may overlap, but the bag that was deleted from the tree decomposition contains all information that is needed to combine solutions on the subgraphs into a feasible solution for the problem on $G$. The internal bag of a tree decomposition thus serves as a separator in the graph and also as an interface between the two induced subgraphs. We can keep decomposing the subgraphs 
by deleting other bags from the subtrees until all subgraphs have size at most $k+1$. Since $k$ is small, solutions to the subgraphs can easily be obtained. The interfaces between the subgraphs also have size at most $k+1$ and hence different solutions to the subgraphs can be efficiently combined into an optimal solution to the problem on original graph $G$.

A class of graphs is said to have bounded treewidth if their treewidth is bounded from above by a constant that does not depend on the size of the graph. Bounded treewidth graphs have tree decompositions of bounded width. For an overview of classes of graphs that have bounded treewidth, we refer to $(27 ; 66)$.

Many problems that are NP-complete on general graphs are solvable in polynomial or even linear time on bounded treewidth graphs $(6 ; 8 ; 9 ; 26 ; 90)$. The most important characterization of $N P$-complete problems that can be solved on graphs of bounded treewidth was derived by Courcelle. In (52), he obtained as a fundamental result that all NP-complete problems that can be expressed in Monadic Second-Order Logic (MSOL) are linear-time solvable on graphs of bounded treewidth, if a bounded width tree decomposition of the graph is given. Algorithms solving these problems typically apply a divide-and-conquer strategy on a bounded width tree decomposition.

\subsection{1 when treewidth is not bounded}

What if we want to solve problems on graphs that do not have bounded treewidth? Does it make sense to use a tree decomposition based approach for solving $N P$-hard problems on these graphs where construction of an optimal tree decomposition is an NP-hard problem in itself? These are some reasonable questions that will be addressed in this subsection.

First of all, graphs that represent real-life settings like communication networks often have low treewidth. Although they are not necessarily part of a bounded treewidth class, they are typically quite sparse and are therefore treelike. An optimal tree decomposition can then often be found in acceptable time bounds and a tree decomposition based approach for solving the problem can be efficiently applied.

Furthermore, if an exact method to find an optimal tree decomposition is not quick enough, other methods (see Section 1.7.2 and 1.7.3) can be used to obtain tree decompositions of reasonably low width. It might very well be the case that the tree decomposition based approach to solve the problem is already fast enough for our purposes when we use this non-optimal tree decomposition.

A third reason for using a tree decomposition based technique to solve $N P$-complete problems is the re-usability of tree decompositions. It happens that several hard problems must be solved on the same graph instance. Once a good tree decomposition of such a graph is available, it can be used over and over for solving multiple problems. In this context, it will probably pay off to spend some more time and effort in constructing a single good tree decomposition. 


\subsection{Dynamic programming on tree decompositions}

Dynamic programming is an algorithm design paradigm that systematically applies the before-mentioned divide-and-conquer strategy. The method was invented in 1953 by applied mathematician Richard E. Bellman. Tree decompositions lend themselves very well for application of dynamic programming algorithms.

Consider a problem on input graph $G$ and suppose a tree decomposition of $G$ is available. By choosing an arbitrary bag as the root of the tree decomposition, one can specify a relation between each pair of bags in the tree decomposition. Any bag $X$ on the unique path from bag $Y$ to the root (including the root itself) is called an ancestor of bag $Y$. If $X$ is an ancestor of $Y$, then $Y$ is a descendant of $X$. If bag $X$ is an ancestor of bag $Y$ and $X$ and $Y$ are connected by an edge, $X$ is said to be a parent of $Y$ and $Y$ a child of $X$. Bags in the tree decomposition that have no descendants are called leaves.

The idea of the dynamic programming approach is to associate to each bag $X$ the subgraph of $G$ that is induced by all vertices in $X$ and its descendants. For each bag in the tree decomposition, one computes a table that contains sufficient information to solve the problem on the subgraph of $G$ corresponding to this bag. The tables are computed in a bottom-up manner, which means that one first has to compute the tables for the leaves. Tree decompositions can be made in such a way that the leaves are sufficiently small, hence computing the tables for the leaves is often trivial. To compute the table for any other bag, one just needs the information from the tables of its children. Tree decompositions can be made in such a way that computing the table for a bag from the tables of its children can be done very efficiently. Since the graph corresponding to the root coincides with graph $G$, the table of the root will contain enough information to determine the solution to the problem on $G$.

The maximum size of a table and the maximum time needed to compute a table are exponential only in the width of the tree decomposition. The dynamic program thus runs in time polynomial in the input size of the graph if and only if the graph has bounded treewidth. Problems that allow for this approach are thus fixed parameter tractable with respect to parameter treewidth.

As an example, let us consider the Weighted Independent Set problem. For this problem we are given a graph $G$ with vertex weights for each vertex. We are looking for a subset $S$ of vertices in $G$ such that the vertices in $S$ are pairwise non-adjacent and the sum of the weights of the vertices in $S$ is maximized. Practical applications of this problem appear among others in information retrieval, signal transmission analysis, scheduling, coding theory and wireless networks. The problem is known to be $N P$-hard for general graphs. For trees however, it is solvable in linear time. In (34), a dynamic programming algorithm on a tree decomposition is presented that runs in time that is only exponential in the width of the tree decomposition. The Weighted Independent Set problem is thus fixed parameter 
tractable with respect to treewidth.

For more details on dynamic programming on tree decompositions and for an overview of other graph decision problems for which this technique has been successfully applied, we refer to respectively (34) and (18).

\subsection{Constructing tree decompositions}

In the previous sections, it became clear that the availability of tree decompositions of low width can considerably speed up the process of solving many NP-complete problems on graphs. Unfortunately, constructing tree decompositions of low (let alone minimum) width is a task that is far from trivial.

The construction of a tree decomposition of width $k$ is obviously at least as hard as deciding whether the treewidth of $G$ is at most $k$, which is already an NP-complete problem. Computing treewidth and constructing tree decompositions of minimum width are $N P$-hard problems even if we restrict the input graphs to graphs of bounded degree (25), cocomparability graphs $(7 ; 71)$, bipartite graphs $(78)$ or the complements of bipartite graphs (7).

Despite these troubling facts, there are many cases for which treewidth can be efficiently determined or approximated.

\subsection{1 exact methods}

Over the last few decades, several graph properties have been shown to facilitate an efficient determination of the graph's treewidth. One of these properties is for the graph to be chordal. Chordal graphs are graphs that are triangulated. Determining the treewidth of a chordal graph allows for a polynomial-time algorithm, since finding the largest clique in a chordal graph is an easy problem. Other graph classes for which the treewidth can be determined in polynomial time include permutation graphs (22), circular-arc graphs (104), circle graphs (78) and distance hereditary graphs $(46)$. In $(41 ; 42)$, an algorithm was presented that determines the treewidth of a graph in time, polynomial in the number of its minimal separators. More examples of polynomial time algorithms for treewidth of graphs from various classes can be found in $(28 ; 29 ; 47 ; 53 ; 77 ; 80)$.

Exact algorithms for treewidth that do not assume or exploit any properties of the input graph require at least exponential time. Based on the results from $(41 ; 42)$, an exact algorithm for treewidth that runs in $\mathrm{O}^{*}\left(1.9601^{n}\right)$ was obtained in (60), where $n$ is the number of vertices in the input graph. For a survey of exact algorithms for NP-hard problems and for explanation on the $\mathrm{O}^{*}()$ notation, we refer to (110). The result from (60) was improved in (61) and (62), decreasing the running time to $\mathrm{O}^{*}\left(1.7549^{n}\right)$. The algorithm however requires 
exponential space. The fastest exact algorithm for treewidth that takes only polynomial space is also from (62) and runs in $\mathrm{O}^{*}\left(2.6151^{n}\right)$ time.

A well studied case is when the integer $k$ is a fixed constant. Constructive algorithms for this case can be useful from a practical point of view, i.e., if the treewidth is at most $k$, a tree decomposition of width at most $k$ must be returned. The first constructive polynomial time algorithm for the fixed parameter problem was found in (7). Although during later improvements upon it the running time of the algorithm was decreased to linear (see e.g., $(20 ; 23 ; 24 ; 86 ; 87 ; 93))$, the complexity of the algorithm still contains a hidden constant that is at least exponential in $k$, restricting its usability only to very small values of $k$ (e.g., $k \leq 4)$. Hence the quest remains for efficient algorithms for the fixed parameter case that are also practical from an implementation viewpoint.

For small graphs the treewidth can often be found in reasonable time using a branchand-bound method. Experiments with constructive methods for treewidth were published in (64) and (102). By terminating the algorithm after a specific time bound and reporting the best solution so far, branch-and-bound algorithms can also be used as heuristics to obtain upper bounds on the treewidth of larger graphs. In Chapter 3 of this thesis, a branch-andbound algorithm will be presented that builds triangulations of the input graph. In the branching step, potential fill-in edges are either added or denied in the triangulation. As will be explained in Chapter 2, a triangulation of width $k$ can easily be transformed into a tree decomposition of width $k$.

\subsection{2 approximation algorithms}

Although constructing an optimal tree decomposition in reasonable time is often impossible, there are many algorithms that approximate the treewidth. Some of them are polynomial even when $k$ is not bounded, others are exponential in $k$. In (21), a polynomial-time approximation algorithm was given that returns a tree decomposition with width at most $\mathrm{O}(\log n)$ times optimal. This result was improved upon in (5) and (44), where polynomial-time approximation algorithms are presented with approximation ratio $\mathrm{O}(\log k)$.

Constant performance ratio approximation algorithms exist for some special graph classes, see for example (43) where a polynomial 2-approximation algorithm was given for the treewidth of graphs that do not contain asteroidal triples. Treewidth can also be approximated within a constant factor for planar graphs. This is a consequence of the polynomial-time algorithm given by Seymour and Thomas (101) for computing the parameter branchwidth in planar graphs, whose value approximates treewidth within a factor of $\frac{3}{2}$. Whether constant ratio approximation algorithms exist for the treewidth problem on general graphs still remains a famous open problem.

Several approximation algorithms exist for treewidth that run in time exponential in $k$, the treewidth of the graph. These are also called fixed parameter approximation algorithms, 
since they are polynomial only when $k$ is fixed. They either give a tree decomposition of width at most $c k$ for some constant $c \geq 1$, or tell that the treewidth is more than $k$. For some examples of them, we refer to $(5 ; 12 ; 87)$.

\subsection{3 heuristics}

Fixed parameter approximation algorithms become less practical when $k$ increases. Moreover, approximation algorithms that are polynomial even when $k$ is unbounded tend to be very time consuming on big graph instances.

When a guaranteed performance ratio is not of primary importance, upper bound heuristics should be considered. They are often much quicker than approximation algorithms and although their performance can theoretically be exponentially bad, experiments have shown that these heuristics often perform very well in practice.

Many upper bound heuristics for treewidth share the same basic structure. A triangulation of the graph is constructed by visiting all vertices of the graph in some order and adding fill-in edges between the neighbors of the vertex at hand that are not yet visited. Different criteria for selecting the next vertex to visit lead to different heuristics. We refer to (83) for computational experiments on several upper bound heuristics.

Another type of upper bound heuristic is based on local search and was proposed in (81). The idea is to start with an arbitrary tree decomposition or triangulation and to reduce its width by making small local changes in the configuration of the bags in the tree decomposition (or cliques in the triangulation). In Chapter 4 of this thesis, we present a local search heuristic that splits the largest bag in a tree decomposition into smaller bags, while maintaining the validity of the tree decomposition. Our experiments show that this technique can lead to significant reduction in the width of tree decompositions resulting from other upper bound heuristics when they are used as a starting solution for the local search heuristic. Combining several types of upper bound heuristics may thus be beneficial.

\subsection{Some applications}

Tree decompositions of bounded width are interesting from a theoretical viewpoint, because all NP-complete problems that can be expressed in MSOL can theoretically be solved in linear time using them, see $(51 ; 52)$. Considering that there are often huge constant factors concealed in their linear running times, algorithms exploiting the bounded width tree decompositions are not always practical. That being said, there are many examples in which tree decompositions have been successfully exploited. In this section, we will highlight a number of them.

In wireless communication networks, frequencies must be assigned to transmitters in 
such a way that interference between the signals of different transmitters is avoided. In (81) and (82), optimal solutions were obtained to some hard frequency assignment problems in wireless networks. This was done by the use of dynamic programming techniques on a tree decomposition of the graph that models interference constraints in the network. In (84), a similar approach was used to solve small and medium-sized instances of the more general class of partial constraint satisfaction problems. The class PCSP contains a diversity of problems, such as generalized subgraph problems, MAX-SAT, Boolean quadratic programs and map coloring problems. For large instances of this class, the technique was shown to provide good lower bounds within reasonable time and memory limits.

A class that can be seen as a generalization of many hard problems (like Independent (Dominating) Set, Induced Bounded Degree Subgraph, Induced $p$-Regular Subgraph, Perfect Matching Cut and $k$-Colorability), is the class of Vertex Partitioning Problems. Given a graph, a Vertex Partitioning problem queries whether its vertices can be partitioned into a number of sets such that the sets comply to some particular conditions. In (105), it was shown that a large class of vertex partitioning problems is fixed parameter tractable when parameterized by treewidth. The FPT-algorithms exploit a bounded width tree decomposition of the input graph.

A dominating set in a graph is a subset $D$ of the vertices such that every vertex not in $D$ is connected via an edge to at least one vertex in $D$. A vertex cover of a graph is a subset $C$ of the vertices such that each edge in the graph is incident to at least one element of $C$. The problem of finding the smallest vertex cover or dominating set in a graph has applications for example in sensor networks and social networks. A tree decomposition based approach enabled the authors in (4) to solve the Vertex Cover problem on planar graphs to optimality. In (2) and (3), tree decomposition based FPT-algorithms are suggested for the Dominating Set problem on planar graphs as well as for several other domination-like problems.

Practical applications of tree decompositions can also be found in network reliability. The reliability of a network measures the probability that communication in the network is possible, given probabilities for each of the elements in the network (e.g., links, routers) to break down. Many problems that arise in this context are $N P$-hard on general graphs. However, information and communication networks like Internet and telephone networks typically have low treewidth, simply because a tree is the cheapest way to connect a set of sites. In (91) and (111), tree decomposition based linear-time algorithms are proposed for several network reliability problems on graphs of bounded treewidth.

Another application that we bring up here concerns probabilistic networks, also called (Bayesian) belief networks. These networks are directed acyclic graphs that represent a set of random variables and their conditional (in)dependencies. They are used in decision support systems and expert systems. The value of a vertex $v$ in the network depends on the value of all vertices that have an arc to $v$. Computing the conditional probabilities of all 
vertices is called probabilistic inference, which is an $N P$-hard problem in general. In (88), an algorithm is presented that solves the problem in $\mathrm{O}\left(n 2^{w}\right)$ time by dynamic programming on a tree decomposition of an auxiliary graph of the network, where $w$ is the width of the tree decomposition.

Recent applications also emerge from the field of Bioinformatics. Consider for example the problem of searching for some queried sequence in a Ribonucleic Acid (RNA) family with pseudoknots. Pseudoknots are secondary structures in RNA containing two stem-loop structures in which half of one stem is intercalated between the two halves of another stem. Searching for queried sequences of RNA in a structure with pseudoknots turns out to be a hard problem when using methods as Hidden Markov Models and Covariance Models. In (103), graphs are used to model both the RNA family with pseudoknots as well as the queried sequence of RNA. The problem of finding the queried sequence segment then boils down to the subgraph isomorphism problem, which is $N P$-hard in itself. However, since a graph modeling an RNA family with pseudoknots typically has treewidth that is only slightly higher than 2 , the problem of recognizing queried sequences of RNA can be efficiently solved using dynamic programming on a tree decomposition of the graph. Searches that required several months with other methods can now be accomplished in days. In (112), some key problems in protein structure prediction were efficiently solved with the help of tree decompositions.

\subsection{Thesis outline}

The basic terminology that will be utilized throughout the thesis is clarified in Chapter 2 . Next to formal definitions of treewidth, tree decomposition and some more notions that will be used throughout the thesis, Chapter 2 also covers some fundamental results that will be assumed as pre-knowledge in the remainder of the thesis.

In Chapter 3, we present a branch-and-bound algorithm that determines the treewidth of a graph and returns a tree decomposition of optimal (minimum) width. Since the algorithm is exact and therefore not efficient, its practical usability is limited to relatively small graph instances only. However, by terminating the algorithm after a fixed time bound, it can serve as a way to obtain lower and upper bounds on the treewidth of larger graphs.

When exact methods fail, it is necessary to resort to approximation algorithms or heuristics. We develop a treewidth upper bound heuristic that will be the subject of Chapter 4 in this thesis. The heuristic utilizes a local search technique and takes as input an arbitrary tree decomposition. It then manipulates the tree decomposition by making a series of local changes to it, while preserving its defining properties. The ultimate goal of the manipulation steps is to obtain a reduction in the width. Implementation of the local search heuristic reveals that the width of tree decompositions (even for those resulting from other upper 
bound heuristics) can often be significantly decreased in an acceptable amount of time.

Subsequently, we turn our attention to the class of planar graphs. This class consists of all graphs that can be embedded in the plane without crossing edges. Planar graphs do not have bounded treewidth and the question whether or not computing the treewidth of planar graphs is $N P$-hard is still unanswered. The treewidth of a planar graph is however related to some other parameters of planar graphs, namely its branchwidth (also holds for general graphs) and the size of its largest square grid-minor. The nature of these relationships is examined more closely in Chapter 5 of this thesis. In this chapter, we also introduce a methodology that can be used to determine the size of a largest square grid-minor for some planar graphs.

In the final chapter of the thesis, Chapter 6, we investigate the problem of excluding a set of graphs as subgraph of input graph $G$ by deleting a (minimum) number of edges from $G$. This problem is $N P$-hard for general graph instances. We show that the problem (depending on the type of subgraphs) is fixed parameter tractable when parameterized by the treewidth. Moreover, we show that an optimal solution to the problem can be efficiently approximated if the input graph is planar. The algorithms in this last chapter employ dynamic programming techniques that are very illustrative for the value of tree decompositions in dealing with $N P$ hard problems.

\subsection{Published material}

Part of the material presented in this thesis has been published or has been accepted for publication in refereed journals or conference proceedings. The following publication is based on Chapter 4 of this thesis.

Stan P. M. van Hoesel and Bert Marchal. Finding good tree decompositions by local search. Electronic Notes in Discrete Mathematics, 32 (2009), pp. 43-50.

An extended version of the work described in Chapter 5 of this thesis has been published in the following publication.

Alexander Grigoriev, Bert Marchal and Natalya Usotskaya. On Planar Graphs with Large Treewidth and Small Grid-Minors. Electronic Notes in Discrete Mathematics, 32 (2009), pp. 35-43.

Finally, Chapter 6 of this thesis forms the source of the following two publications.

Alexander Grigoriev, Bert Marchal and Natalya Usotskaya. Algorithms for the Minimum Edge Cover of H-Subgraphs of a Graph. In Proc. of the 36th Conference on Current Trends in Theory and Practice of Computer Science (SOFSEM 2010), Lecture Notes in Computer Science, 5901, Springer (2010), pp. $352-464$.

Alexander Grigoriev, Bert Marchal, Ioan Todinca and Natalya Usotskaya. A note on planar graphs with large width-parameters and small grid-minors. Accepted in Discrete Applied Mathematics, 2012. 


\section{Chapter 2}

\section{Preliminaries}

In this chapter we clarify the majority of the terminology that will be used throughout this thesis. Some terms that are utilized only occasionally will be introduced later, at the point where they are needed. We commence with some terms and notions that regard standard graph theory. After that some terminology will be stated concerning the subject of planar graphs and subsequently the notion graph minor will be explained. We round this chapter off with formal definitions of treewidth, tree decompositions and some related terms.

\subsection{Graph terminology}

A graph $G$ is a pair $(V(G), E(G))$, where $V(G)$ is a finite set of vertices and $E(G)$ is a (multi)set of edges. Figure 2.1 displays a graph on ten vertices and fifteen edges, known as the Petersen graph. If there is no ambiguity about which graph $G$ we deal with, we will refer to

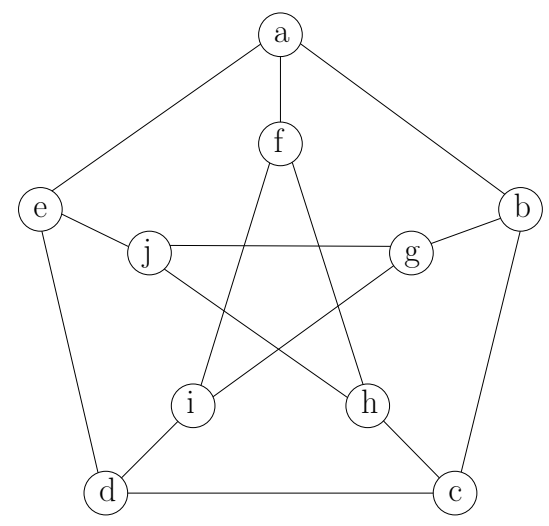

Figure 2.1: the Petersen graph 
$V(G)$ and $E(G)$ simply as $V$ and $E$. Following the convention in graph theory, we use $n(G)$ (or simply $n$ ) to denote the number of vertices in $G$ and $m(G)$ (or simply $m$ ) to denote the number of edges in $G$, i.e., $n=|V|$ and $m=|E|$. An edge $e \in E$ is a two-element (multi)-set of vertices from $V$, i.e., $e \in V \times V$. If an edge $e$ joins two vertices, then these vertices are called the end vertices of $e$. If $u$ and $v$ are the end vertices of the edge $e$, then $e$ is formally denoted by $\{u, v\}$. For the sake of readability, we will refer to the edge $\{u, v\}$ simply as $u v$ in this thesis. Two edges are disjoint if they have no end vertex in common. An edge $e$ and an end vertex of $e$ are said to be incident to one another. Two vertices that are joined by an edge are called adjacent vertices. In the Petersen graph as depicted in Figure 2.1, vertices $a$ and $b$ are adjacent and they are the end vertices of edge $a b$. In this example, vertex $f$ is incident to edges $a f, f h$ and $f i$. The open neighborhood $N(v)$ of a vertex $v \in V$ in $G$ is the set of vertices adjacent to $v$ in $G$. In the Petersen graph, e.g., $N(f)=\{a, i, h\}$.

The degree $d(v)$ of a vertex $v \in V$ is the number of edges incident to $v$ in $G$. The minimum degree over all vertices in $G$ is denoted by $\delta(G)$, i.e., $\delta(G)=\min _{v \in V} d(v)$. Similarly, $\Delta(G)$ denotes the maximum degree in $G$, i.e., $\Delta(G)=\max _{v \in V} d(v)$. For the graph in Figure 2.1, $\delta(G)=\Delta(G)=3$. An edge $e \in E$ is called directed if the pair of vertices denoting the edge is ordered and $e$ is said to be undirected if it is represented by an unordered vertex pair. A graph $G$ is undirected if all of its edges are undirected. An edge is called a self-loop if its two end vertices coincide. When $E(G)$ is a set rather than a multi-set and does not contain self-loops, $G$ is said to be a simple graph. The graph in Figure 2.1 is simple and undirected. Unless stated otherwise, the graphs in this thesis are both simple and undirected.

A path in a graph $G$ is a sequence of vertices from $V$ such that each vertex in the sequence is adjacent to the next vertex in the sequence. For finite paths, the first vertex is called the start vertex and the last vertex is called the end vertex. All other vertices in a path are called internal vertices. A cycle is a path for which the start vertex and the end vertex coincide. A path with no repeated vertices is called a simple path, and a cycle with no repeated vertices or edges aside from the necessary repetition of the start and end vertex is a simple cycle. A graph is acyclic if it does not contain any cycle. The length of a path is the number of edges in the path, counting multiple edges multiple times. The sequence $a f h j g b$ is a simple path of length 5 in the Petersen graph from Figure 2.1. Two paths in a graph are independent if they have no internal vertices in common. The distance between two vertices in a graph $G$ is the length of the shortest path in $G$ connecting the two vertices. The maximum distance between two vertices in the Petersen graph equals 2 .

If $V^{\prime} \subseteq V$ and $E^{\prime} \subseteq E \cap\left(V^{\prime} \times V^{\prime}\right)$, then $G^{\prime}=\left(V^{\prime}, E^{\prime}\right)$ is a subgraph of $G=(V, E)$, written as $G^{\prime} \subseteq G$. If $G^{\prime}$ is a subgraph of $G$, then $G$ is a supergraph of $G^{\prime}$. a subgraph $G^{\prime}$ of $G$ is said to be induced by $V^{\prime}$ if $E^{\prime}$ contains all edges from $E$ for which both end vertices are in $V^{\prime}$. The subgraph of $G=(V, E)$ that is induced by $V^{\prime} \subseteq V$ is denoted by $G\left[V^{\prime}\right]$. For $v \in V$, we denote by $G \backslash v$ the graph that is obtained from $G$ by deleting $v$ and all edges incident to $v$, 
i.e., $G \backslash v=G[V \backslash\{v\}]$. For $e \in E$, by $G \backslash e$ we denote the graph that results from $G$ when we delete edge $e$.

A clique in $G$ is a subset of $V$ for which all vertices are pairwise adjacent. A clique $S$ in $G$ is called a maximal clique if no strict superset of $S$ is a clique. A clique $S$ in $G$ is called a maximum clique if there is no clique in $G$ that has bigger size. The size of a maximum clique in $G$ is denoted by $\omega(G)$. A graph on $n$ vertices that forms a clique on $n$ vertices is called a complete graph and is denoted by $K_{n}$. An independent set in $G$ is a subset of $V$ in which the vertices are pairwise non-adjacent. Maximal and maximum independent sets can be defined analogously to maximal and maximum cliques. The set $\{a, f\}$ is a maximal (and maximum) clique and set $\{b, e, h, i\}$ is a maximal (and maximum) independent set in the Petersen graph from Figure 2.1.

A graph is said to be connected if there is a path between each pair of distinct vertices of the graph. The Petersen graph is an example of a connected graph. A connected component $S$ of $G$ is a maximal subgraph of $G$ that is connected, i.e., each supergraph of $S$ that is a subgraph of $G$ is disconnected. Most problems on graphs can be cracked by solving them separately on each connected component of the graph. It is for this reason that we only consider input graphs in this thesis that have exactly one connected component, i.e., we assume that our input graphs are connected. Connected graphs that are acyclic are called trees.

\subsection{Planar graphs}

A graph $G$ is called a planar graph if it can be drawn in the plane in such a way that its edges intersect only at shared end vertices. A drawing of a planar graph $G$ in the plane is called a planar embedding of $G$. The graph from Figure 2.1 is non-planar, since it can not be embedded in the plane without crossing edges. The regions that are bounded by the edges in a planar embedding of a planar graph are called faces. If we embed a planar graph on the plane, there is always one face that is unbounded. This is called the outer face. All other faces are called inner faces. The number of faces in a planar embedding is denoted by the letter $f$. The next theorem implies that for a connected planar graph $G$, the parameter $f$ does not depend on the embedding of $G$.

Theorem 2.2.1. (Euler's formula) Let $G$ be a connected planar graph with $n$ vertices and $m$ edges. Then for every planar embedding of $G$ it holds that

$$
n-m+f=2 .
$$

A graph $G$ is called outerplanar (or 1-outerplanar) if there is a planar embedding of $G$ in which each vertex is incident to the outer face. For $k>1$ a planar embedding is $k$ outerplanar if removing the vertices incident to the outer face results in a $(k-1)$-outerplanar 
embedding. A graph $G$ is $k$-outerplanar if there exists a $k$-outerplanar embedding of $G$. The notion of $k$-outerplanar graphs was introduced by Baker, see (11). The smallest integer $k$ for which $G$ is $k$-outerplanar is called the outerplanarity index of $G$. As an example, the graph in Figure 2.2 has outerplanarity index 2, since there is no 1-outerplanar embedding of $G$.
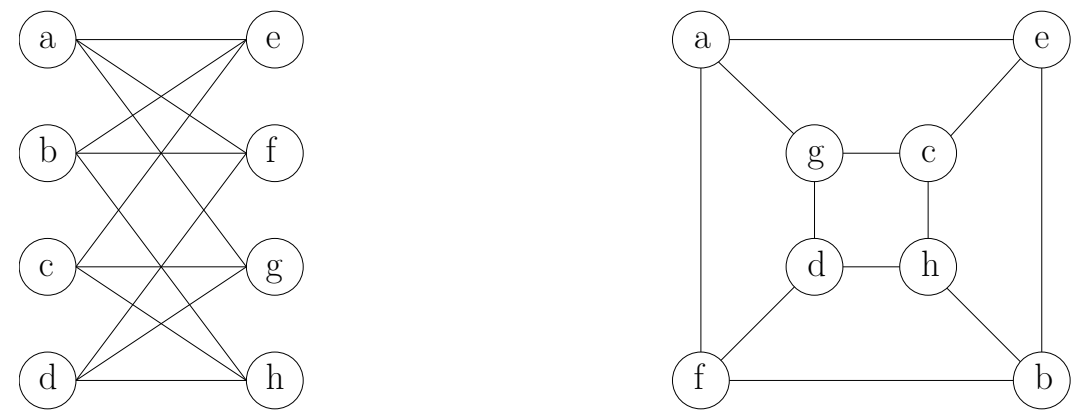

Figure 2.2: a planar graph $G$ and a 2-outerplanar embedding of $G$

In (15), it is shown that the outerplanarity index of an arbitrary planar graph can be determined in polynomial time. The following theorem is from (75):

Theorem 2.2.2. Given a planar graph $G$, the outerplanarity index $k$ of $G$ and a $k$-outerplanar embedding of $G$ can be found in $O\left(n^{2}\right)$ time.

The following theorem from (27) relates the outerplanarity index of a graph to its treewidth:

Theorem 2.2.3. The treewidth of a $k$-outerplanar graph is at most $3 k-1$.

\subsection{Graph minors}

We first explain the notion of an edge contraction in a graph. While doing so, we follow the terminology from (57). Subsequently, we give a formal definition of a graph minor. Minors can be obtained from a graph by a series of vertex deletions, edge deletions and edge contractions in any order. They play an important role in the characterization of many families of graphs.

Informally, contraction of edge $e=u v$ replaces vertices $u$ and $v$ by a new vertex that is adjacent to all neighbors of both $u$ and $v$. In this thesis, we restrict ourselves to simple graphs and hence we enforce that after contraction, the new vertex is connected to its neighbors via a single edge and not via a multi-edge. This is illustrated in Figure 2.3.

As is formalized in the next definition, we use G/e to denote the graph that is obtained from $G$ by contracting edge $e$. 


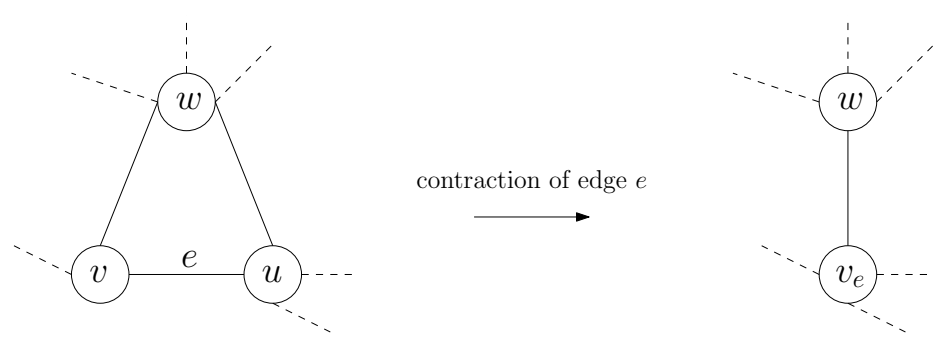

Figure 2.3: Before contraction both $u$ and $v$ are adjacent to $w$. After contraction of $e$, the new vertex $v_{e}$ is connected to $w$ only via a single edge.

Definition 2.3.1. Let be given a graph $G=(V, E)$ and let $e=u v \in E$. Then G/e is the graph $\left(V^{\prime}, E^{\prime}\right)$ that results from $G$ after contracting edge e, where $V^{\prime}=(V \backslash\{u, v\}) \cup\left\{v_{e}\right\}$ (with $v_{e}$ the new vertex) and

$$
E^{\prime}=\{x y \in E \mid\{u, v\} \cap\{x, y\}=\varnothing\} \bigcup\left\{v_{e} x \mid u x \in E \backslash\{e\} \text { or } v x \in E \backslash\{e\}\right\} .
$$

Two graphs $G$ and $H$ are said to be isomorphic if there exists a bijection $f:=V(G) \rightarrow$ $V(H)$ such that any two vertices $u$ and $v$ are adjacent in $G$ if and only if $f(u)$ and $f(v)$ are adjacent in $H$.

Definition 2.3.2. A graph $M$ is a minor of $G$, written as $M \leq G$, if $M$ is isomorphic to a graph that can be obtained from a subgraph of $G$ by doing a number (zero or more) of edge contractions.

Figure 2.4 shows a graph $G$, a subgraph $H$ of $G$ and a minor $M$ of $G$. $M$ can be obtained from $H$ by contracting the dashed edges.

A family $\mathscr{F}$ of graphs is said to be closed under taking graph minors if for every graph $G$ in $\mathscr{F}$, all minors of $G$ are also element of $\mathscr{F}$. Planar graphs and the family of bounded treewidth graphs are examples of graph-minor-closed families.

We say that we subdivide an edge $e=u v$ in $G$ if we add a new vertex $w$ to $G$ and replace the edge $u v$ by edges $u w$ and $v w$. A subdivision of $G$ can be obtained from $G$ by a series of subdivisions of the edges of $G$. If a graph $M$ has a subdivision that is isomorphic to a subgraph of $G$, then $M$ is called a topological minor of $G$. Since in Figure 2.4, graph $H \subseteq G$ is a subdivision of $M, M$ is a topological minor of $G$.

In 1930, Kuratowski (85) provided a characterization of planar graphs in terms of two forbidden topological minors; the complete graph $K_{5}$ and the complete bipartite graph $K_{3,3}$. Seven years later in (108), Wagner proved that a graph is planar if and only if it does not have $K_{5}$ or $K_{3,3}$ as a regular minor. This led him to the bold conjecture that the set of forbidden minimal minors of any infinite graph-minor-closed family of graphs is finite. A proof of the theorem by Robertson and Seymour was completed in 2004 with the publication 


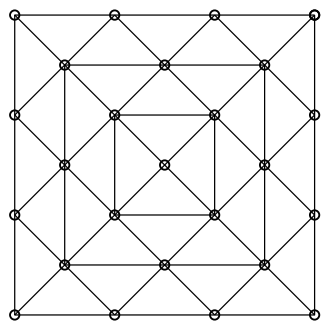

G

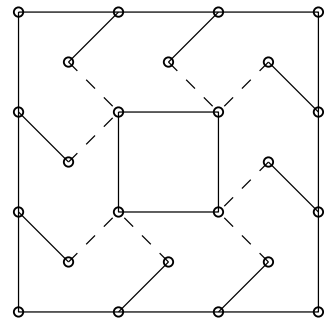

$\mathrm{H}$

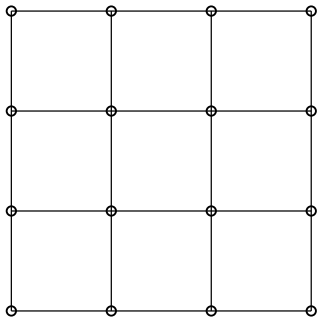

$\mathrm{M}$

Figure 2.4: graph $G$, subgraph $H \subseteq G$ and minor $M \leq G$.

of the last in a series of twenty papers (see, among others, $(94 ; 95 ; 96)$ ) running to over 500 pages and spanning almost 20 years.

\subsection{Treewidth}

In this section, we formally define treewidth and a few related terms. The notions of treewidth and tree decomposition were introduced by Robertson and Seymour in (94). Apart from their definitions, we introduce some fundamental lemma's regarding treewidth that are essential for some lemma's and theorems in later chapters of the thesis.

A tree decomposition of a graph $G$ is a pair $(T, X)$, where $T$ is a tree and $X=\left(X_{t}: t \in\right.$ $V(T))$ is a family of subsets of $V(G)$, with the following properties:

- $\cup_{t \in V(T)} X_{t}=V(G)$.

- $\forall u v \in E(G), \exists t \in V(T)$ such that $\{u, v\} \subseteq X_{t}$.

- For $t, t^{\prime}, t^{\prime \prime} \in V(T)$, if $t^{\prime}$ is on the unique path in $T$ between $t$ and $t^{\prime \prime}$ then $X_{t} \cap X_{t^{\prime \prime}} \subseteq X_{t^{\prime}}$.

For $t \in V(T)$, the set $X_{t}$ is also referred to as a bag of the tree decomposition $(T, X)$. The width of the tree decomposition $(T, X)$ is $\max _{t \in V(t)}\left(\left|X_{t}\right|-1\right)$.

Definition 2.4.1. The treewidth $t w(G)$ of graph $G$ is the minimum width over all tree decompositions of $G$.

The following useful fact about tree decompositions originates from (19).

Lemma 2.4.2. Let $(T, X)$ be a tree decomposition of graph $G$. Then for any clique $S$ in $G$, there is a bag $X_{t}$ in $(T, X)$ for which $S \subseteq X_{t}$.

The relation between treewidth of $G$ and the treewidth of a minor of $G$ can be captured in the following lemma, which is due to Bodlaender (27). 
Lemma 2.4.3. If $M \leq G$, then $t w(M) \leq t w(G)$.

A lot of research has been dedicated to the fixed parameter case for treewidth, i.e., check whether the treewidth of a graph is at most some constant $w$ and if so, return a tree decomposition of width at most $w$. For an overview of this work we refer to (30). Finally, in (23) the following result was obtained.

Theorem 2.4.4. Given a graph of treewidth at most $w$, a tree decomposition of width at most $w$ can be obtained in linear time.

From a practical viewpoint the algorithm is only useful for low values of $w$ because of a big hidden constant in the $\mathrm{O}($ )-notation in the running time of the linear algorithm.

An edge which joins two vertices of a cycle but is not itself an edge of the cycle is called a chord of that cycle. A chordless cycle in $G$ is an induced cycle in $G$, i.e., a cycle that forms an induced subgraph of $G$. A graph is called chordal (or triangulated) if it does not contain chordless cycles of length greater than 3, i.e., if all induced cycles in the graph are 3-cycles (also called triangles). A graph can be transformed into a chordal graph by adding edges to it up to the point where every cycle contains a chord. Edges that are added to achieve chordality are called fill-in edges. In this thesis, we denote the set of fill-in edges by $F$.

Definition 2.4.5. A graph $H=(V, E \cup F)$ is called a chordalization (or triangulation) of $G=(V, E)$ if $H$ is chordal.

The width of a chordal graph $H$ is equal to $\omega(H)-1$, i.e., the size of the largest clique in $H$ minus one. Using the notion of chordalization, treewidth can be alternatively defined in the following way.

Definition 2.4.6. The treewidth $t w(G)$ of graph $G$ is the minimum width over all chordalizations of $G$.

The following result concerning chordal graphs comes from (98).

Lemma 2.4.7. Given a chordal graph $G$, the size of a largest clique in $G$ and hence $t w(G)$ can be determined in polynomial time.

An ordering of the vertex set $V$ is a bijection $\alpha:\{1,2, \ldots, n\} \longleftrightarrow V$. If $\alpha$ is an ordering on $n$ vertices, then we will also refer to $\alpha$ as $\alpha(1) \alpha(2) \ldots \alpha(n)$. A chordalization of $G=(V, E)$ can be obtained using vertex ordering $\alpha$ of $V$ by application of Algorithm 2.1. In words, we run through the vertex ordering and turn the higher ordered neighbors of the vertex at hand into a clique by adding fill-in edges to $G$. Note that the added fill-in edges also define neighbor relations in subsequent steps of the algorithm.

The process of turning the higher ordered neighbors of vertex $\alpha(i)$ into a clique is often called the elimination of vertex $\alpha(i)$. In the context of chordalizations, vertex orderings 


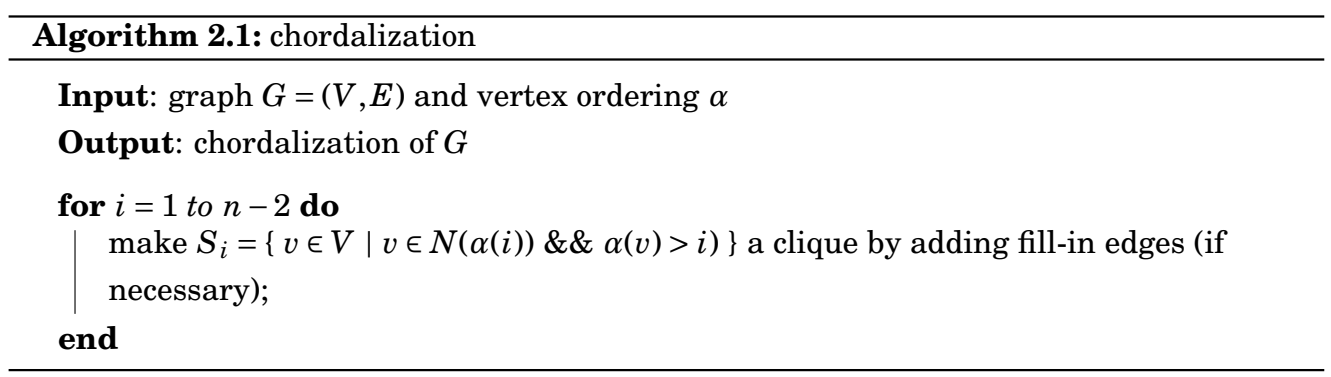

are therefore often referred to as elimination orderings. An elimination ordering $\alpha$ on the vertices of $G$ is called perfect if during Algorithm 2.1 no fill-in edges are added to $G$, i.e., if for all vertices $v \in V$ the set of higher ordered neighbors already forms a clique in $G$.

As an example, a graph is depicted in Figure 2.5 with a chordalization of the graph that is obtained using an elimination ordering on its vertices. Fill-in edges $b d, b f, d h, f h, d f$ are added during elimination of respectively the vertices $a, c, i, g, b$. While eliminating the last four vertices in the ordering, no fill-in edges need to be added.
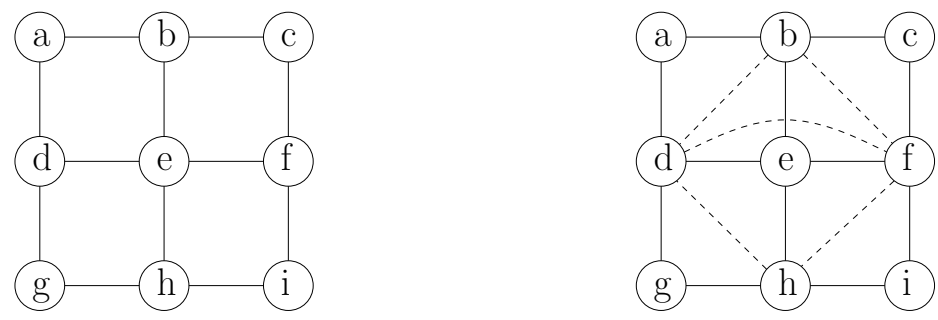

Figure 2.5: graph $G$ and chordalization $H$ of $G$ that is obtained via elimination ordering $\alpha=$ acgibdefh.

A vertex $v$ in a graph $G$ is called simplicial if $N(v)$ induces a clique in $G$. It was shown in (89) that every non-empty chordal graph contains at least one simplicial vertex. Another classical fact is that chordal graphs are recursively simplicial, i.e., they contain a simplicial vertex and after removing this simplicial vertex, the subgraph is still simplicial. A perfect elimination ordering $\alpha$ of a chordal graph $G$ can therefore be obtained by repeatedly selecting a simplicial vertex of $G$ as the next vertex in $\alpha$ and deleting it from $G$.

\subsection{Tree decompositions and chordalizations}

In this thesis, we will use both tree decompositions and chordalizations as structures to bound and determine the treewidth of graphs. However, the two terms are closely related 
in the sense that if we are given a graph $G$ and a tree decomposition of $G$ of width $k$, then it is easy to construct a chordalization of $G$ of width $k$. Algorithm 2.2 shows how this can be done.

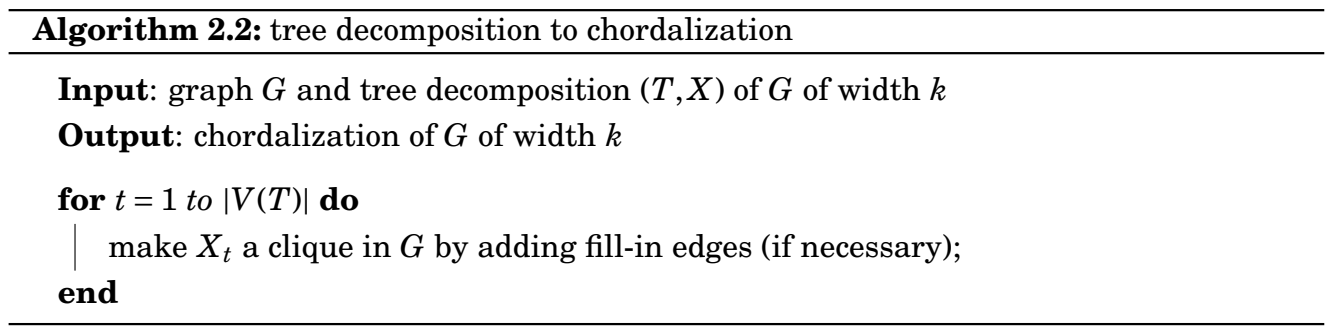

Vice-versa, a chordalization of $G$ can be used to construct a tree decomposition of $G$ of the same width. One way to do this is by application of Algorithm 2.3. Note that this algorithm assumes a perfect elimination ordering (p.e.o.) of the chordalization $H$ of $G$. Such a p.e.o. can be obtained using simplicial vertices as described above. The tree decomposition and

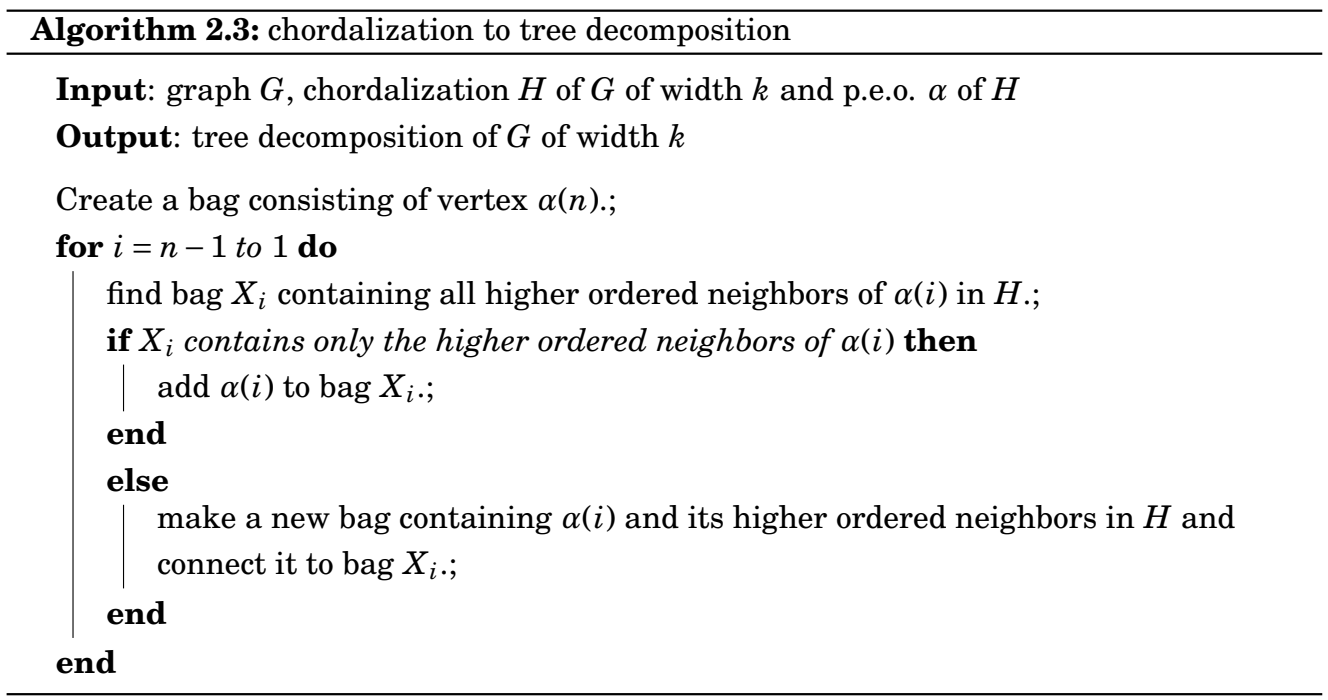

chordalization of the Petersen graph as depicted in Figure 1.3 can be constructed using one another by application of Algorithms 2.2 and 2.3. 



\section{Chapter 3}

\section{Branch and Bound}

This chapter deals with an exact method to determine the treewidth of graphs. The method that will be presented is a branch-and-bound algorithm that operates directly on the input graph and ultimately realizes chordalizations of the graph by adding fill-in edges to it. It is thus constructive in the sense that it not only determines the treewidth of a given graph instance, but also returns a chordalization of optimal (minimum) width. By application of Algorithm 2.3, such an optimal chordalization can be used to construct an optimal tree decomposition of the graph instance.

The content of this chapter is based on cooperation with Stan van Hoesel and Hans Ensinck.

\subsection{Introduction}

Finding chordalizations or tree decompositions of minimum width has been a central issue in algorithmic graph theory during the last decades. Its importance lies in the fact that many $N P$-hard problems can be solved efficiently using low-width tree decompositions with dynamic programming type methods. Examples of such problems are Vertex Coloring, Independent Set, and even Hamiltonian Cycle.

Courcelle (51) has shown that for each graph property that can be formulated in Monadic Second Order Logic (MSOL), there is a polynomial time algorithm that verifies if the property holds if a bounded width tree decomposition of $G$ is available. Such algorithms operate in two steps. First a tree decomposition is constructed and then the problem is solved on this tree decomposition. The last step is usually done by some dynamic program for which both running time and memory space consumption are exponential in the width of the tree decomposition, but polynomial in the size of the graph.

Among the first authors applying this technique are Bern et al. (13). For other papers using this technique on a variety of problems, see for instance Wimer et al. (109) for some 
of the pioneering papers with this type of algorithm, Arnborg and Proskurowski (8), Lauritzen and Spiegelhalter (88) for the inference problem in probabilistic networks, Telle and Proskurowski (105) for several vertex partitioning problems, Koster et al. (84) for partial constraint satisfaction problems (in particular frequency assignment problems) and Bodlaender and Koster (34) for the weighted independent set problem. See Arnborg (6) and Bodlaender (25) for surveys on treewidth algorithms and algorithms for intractable problems that are efficient when restricted to graphs of bounded treewidth.

Several graph classes admit polynomial time algorithms for determining their treewidth, e.g.chordal graphs, permutation graphs, circular arc graphs, circle graphs and distance hereditary graphs. The problem, however, when given an arbitrary graph $G$ and an integer $k$, to determine whether the treewidth of $G$ is at most $k$ is $N P$-complete (7).

Most constructive methods focussing on upper bounding the treewidth of graphs are constant factor approximation algorithms or heuristics, see Section 1.7.2 and 1.7.3. The majority of exact algorithms for treewidth is non-constructive, see Section 1.7.1. There is however quite some recent literature focussing on constructive exact methods for determining the treewidth of a graph. These algorithms use either dynamic programming or branch-andbound. Bodlaender et al. (32) give an overview on such algorithms and their theoretical and practical implications. Shoikhet and Geiger (102) constructed an algorithm that takes as input a graph $G$ and an integer $k$. By building sets of minimal separators and so called fragments of $G$ and by applying dynamic programming techniques, the algorithm returns an optimal chordalization of $G$ or a valid statement that the treewidth of $G$ is larger than $k$. Branch-and-bound is used by Gogage and Dechter (64) and in Bachoore and Bodlaender (10), among others. Their methods build perfect elimination orderings by adding vertices one by one in the branching process. Gogate and Dechter introduced a processing method to kill nodes using exchangeability of neighbors in the orderings. Bachoore and Bodlaender (10) added processing based on vertex disjoint paths between vertices. Their methods are practically suitable for graphs of up to 100 vertices and treewidth of no more than 10 .

In this chapter, a new constructive algorithm for determining the treewidth of a graph is introduced. More specifically, a branch-and-bound algorithm is presented that uses a branching scheme in which fill-in edges are added or forbidden. By exploiting the knowledge about forbidden edges in any node of the branch-and-bound tree, new lower bound techniques for the treewidth are developed. Moreover, new processing rules are applied to limit the number of nodes that need to be visited in the branch-and-bound tree.

The remainder of this chapter is organized in the following way: in Section 3.2, some preliminaries and definitions are introduced, part of which will be used exclusively in this chapter. Section 3.3 attends to a special graph structure, the so called necklace, that forms the basis of several processing rules and lower bounds. Section 3.4 then describes the facets of branching and bounding in the algorithm. In Section 3.5, a number of processing rules 
and some graph reduction techniques are introduced. A selection of the ideas presented in this chapter have been implemented and the resulting algorithm has been tested on several classes of graphs. The results of these experiments are presented in Section 3.6. Finally, conclusions and some directions for further research can be found in Section 3.7.

\subsection{Preliminaries}

Section 2.4 describes that a chordalization $H=(V, E \cup F)$ of $G=(V, E)$ can be obtained via an elimination ordering $\alpha$ on vertex set $V$ by application of Algorithm 2.1. The following folklore fact provides a way to determine the width of such a chordalization $H$.

Lemma 3.2.1. Let $H=(V, E \cup F)$ be the chordalization of $G$ that is obtained via elimination ordering $\alpha$ by application of Algorithm 2.1. Then the width of $H$ is equal to the maximum over all vertices $v \in V$ of the number of higher ordered neighbors of $v$ in $\alpha$ at the moment that $v$ is eliminated.

Proof. The width of chordal graph $H$ is equal to the size of its maximum clique minus one. Consider the higher ordered neighbors in $\alpha$ of any vertex $v$ at the moment $v$ is eliminated. Since $v$ will form a clique in $H$ together with these neighbors it follows that the maximum number of the higher ordered neighbors is a lower bound for the width of $H$. To see that the maximum clique in $H$ is induced by some vertex $v$ and its higher ordered neighbors in $\alpha$ at the moment of $v$ 's elimination, let $C$ be a maximum clique in $H$ and let $w$ be the element from $C$ that has the lowest order in $\alpha$. After $w$ 's elimination, no edges are added to $H$ that are incident to $w$. Also, during elimination of $w$, no edges incident to $w$ are added to $H$. Therefore, all other elements of $C$ (which are all higher ordered than $w$ ) are already incident to $w$ at the moment $w$ is eliminated.

A perfect elimination ordering of a chordal graph can be found by recursively selecting simplicial vertices in the graph. By Lemma 3.2.1, the treewidth of a chordal graph thus equals the maximum degree of a simplicial vertex at the moment it is selected. This leads to the following trivial result:

Lemma 3.2.2. Determining the treewidth of a chordal graph can be done in polynomial time.

The algorithm described in this chapter builds chordalizations of some input graph $G=$ $(V, E)$ by adding edges to it and by forbidding vertex pairs to be edges in the chordalization of $G$.

In the remainder of this chapter, the set $B=E \cup F$, the original set of edges in $G$ plus the added edges, will be denoted as the set of black edges, and the set $R$, the vertex pairs forbidden in a chordalization of $G$, will be denoted as the set of red edges. The set of vertex 
pairs that are neither in $R$ nor in $B$, will be denoted as the set $W$ of white edges. Given input graph $G=(V, E)$, nodes in the branch-and-bound tree thus correspond to graphs $S=$ $(V, B, R)$, where $E \subseteq B$, and $B, R$, and $W$ form a partition of the vertex pairs of $G$.

Definition 3.2.3. $S^{\prime}=\left(V, B^{\prime}, R^{\prime}\right)$ is called an extension of $S=(V, B, R)$ if $B \subseteq B^{\prime}$ and $R \subseteq R^{\prime}$.

An extension of $S=(V, B, R)$ can thus be obtained from $S$ by making some white edges black and/or red.

Definition 3.2.4. $S^{\prime}=\left(V, B^{\prime}, R^{\prime}\right)$ is called a chordalization of $S=(V, B, R)$ if $S^{\prime}$ is an extension of $S$ and $H=\left(V, B^{\prime}\right)$ is chordal.

Note that if $S^{\prime}$ is a chordalization of $S$ and $S$ is an extension of $G$, then $S^{\prime}$ is also a chordalization of $G$.

Definition 3.2.5. The treewidth of $S=(V, B, R)$ is the minimum width over all chordalizations $S^{\prime}$ of $S$. If $S$ has no chordalization, then $t w(S)=\infty$.

In Figure 3.1, an example is given of a graph that has no chordalization.

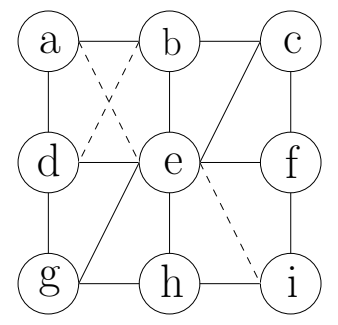

$$
\begin{aligned}
& E \cup F: \\
& R:
\end{aligned}
$$

Figure 3.1: Depicted is the graph $S=(V, B, R)$. In all supergraphs of $S$, the cycle abeda is chordless. Therefore $t w(S)=\infty$.

Let it be clear that the red edges in $S=(V, B, R)$ are not real edges, so whenever a cycle, a path or the degree of a vertex in $S$ are mentioned, they refer to the cycle, path or degree of the vertex in the corresponding graph $H=(V, B)$.

This section ends by introducing a lemma concerning chords in chordal graphs.

Lemma 3.2.6. Let $C$ be a cycle of length at least 4 in a chordal graph $G$ and let $v$ be a vertex on $C$. Then at least one of the following statements is true:

- The neighbors of $v$ on $C$ are connected by a chord.

- There is a chord of C incident to $v$. 
Proof. Proof by contradiction. Suppose there is a vertex $v$ on $C$ such that the neighbors $u$ and $w$ of $v$ on $C$ are not connected by a chord and no chords of $C$ are incident to $v$. Then consider the shortest cycle $C^{\prime}$ in $G$ containing path $u v w$ and using only chords or edges of $C$. Since there are no chords in $C$ incident to $v$ and $u w$ is not a chord of $C$, cycle $C^{\prime}$ must be a chordless cycle with length at least 4 , contradicting the chordality of $G$.

\section{3 the Necklace structure}

Both processing rules and bounding methods in this chapter depend heavily on the presence of a certain structure in the graph. This structure, which will be called a necklace, is introduced in this section and several lemmas are presented concerning chordalizations of graphs $S=(V, B, R)$ that contain necklaces.

Definition 3.3.1. A path $P=v_{1} \ldots v_{n}$ of black edges in $S=(V, B, R)$ for which $v_{i} v_{i+2} \in R$ for $i=1, \ldots, n-2$ is called a necklace in $S$.

If extension $S=(V, B, R)$ of $G=(V, E)$ contains a necklace, several conclusions can be drawn about possible chordalizations of $S$. They are summarized in the following set of lemmas that will later on be used to process graph $S$ in a node of the branch-and-bound tree.

Lemma 3.3.2. Let $P=v_{1} \ldots v_{n}$ be a necklace in $S=(V, B, R)$. Then in any chordalization of $S$, there are no black edges between vertices of $P$ that are non-adjacent on $P$.

Proof. Suppose that in some chordalization $S^{\prime}$ of $S$, there is a black edge between two vertices $v_{i}$ and $v_{j}$ that have distance of at least 3 on $P$. Select $v_{i}$ and $v_{j}$ as close as possible to each other on $P$. Then in $S^{\prime}, v_{i} \ldots v_{j} v_{i}$ is a cycle of length at least 4 , without any black chords. This contradicts the fact that $S^{\prime}$ is a chordalization of $S$.

Lemma 3.3.2 is illustrated in Figure 3.2. The next lemma concerns a vertex in $S$ that is connected to two vertices on a necklace in $S$.

Lemma 3.3.3. Let $P=v_{1} \ldots v_{n-1}$ be a necklace in $S=(V, B, R)$ and let $v_{n}$ be connected to both $v_{1}$ and $v_{n-1}$. Then in every chordalization of $S, v_{i} v_{n}$ will be a black edge for $i=1, \ldots, n-1$.

Proof. By Lemma 3.3.2 the vertices of $P$ will induce a path in the chordalization. Therefore, all chords of the cycle $C=v_{1} \ldots v_{n} v_{1}$ in the chordalization must be incident to $v_{n}$. This implies that in the chordalization, there are chords $v_{i} v_{n}$ for $i=2, \ldots, n-2$ in $C$.

An illustration of Lemma 3.3.3 can be found in Figure 3.3. The last lemma of this chapter concerns a special type of cycle in $S=(V, B, R)$. 
necklace in $S$

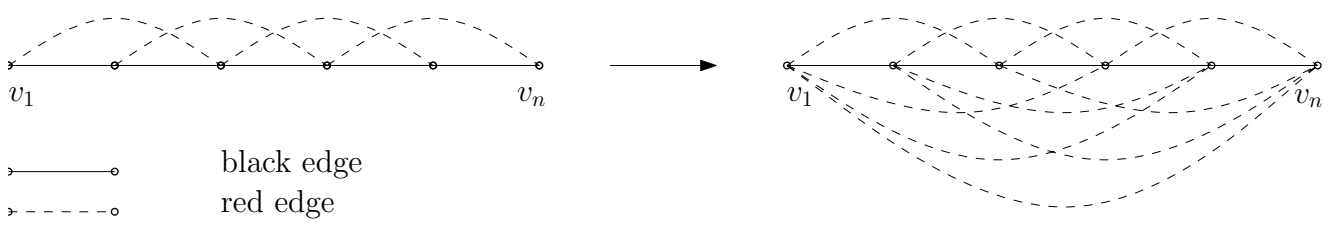

Figure 3.2: In any chordalization of $S$, there are no black edges between vertices of necklace $P$ that are non-adjacent on $P$.

closed necklace in $S$

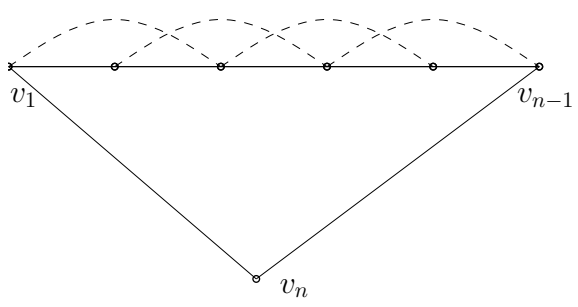

black edge red edge any triangulation of $S$

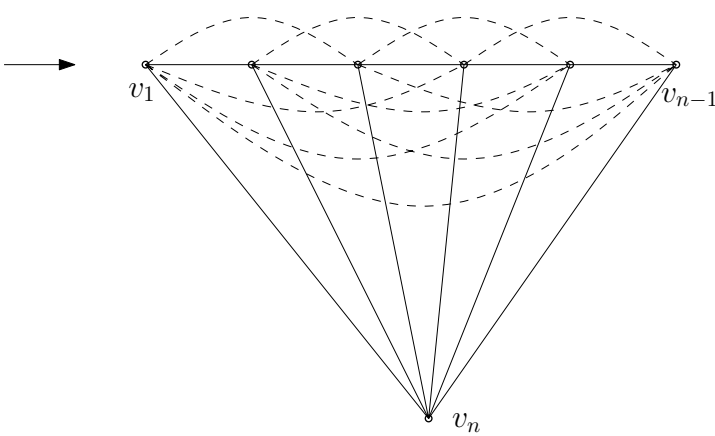

Figure 3.3: Let $P=v_{1} \ldots v_{n-1}$ be a necklace and $C=v_{1} \ldots v_{n} v_{1}$ be a cycle in $S$. Then in all chordalizations of $S$, cycle $C$ has exactly $n-3$ chords, all incident to $v_{n}$.

Lemma 3.3.4. Let $C=v_{1} \ldots v_{n} w v_{1}$ be a cycle of length at least 4 in $S=(V, B, R)$. If vertex $w$ is connected with red edges to all vertices in $C$ except its neighbors, then in all chordalizations of $S$, the edge $v_{1} v_{n}$ will be a black edge.

Proof. If in a chordalization of $S$, cycle $C$ has no chords incident to $w$, then by Lemma 3.2.6, the neighbors of $w$ on $C$ must form a chord in the chordalization. 


\subsection{Branch-and-Bound algorithm for treewidth}

Branch-and-bound is the most widely used tool for solving large scale NP-hard combinatorial optimization problems. It is used here in a fairly standard way. Branch-and-bound algorithms rely on a systematic enumeration of all possible solution candidates. In this study, this implies that all potential chordalizations of the graph will, either explicitly or implicitly, be considered.

The branch-and-bound tree consists of nodes each representing a subproblem of the original problem. The tree is built by starting with one node (the root), which represents the original problem, with $S=(V, E, \varnothing)$. The subproblem in a node is to find the minimum width over all chordalizations of $S$, or equivalently, to determine the treewidth of $S$. In the process, nodes are split by extending graph $S$, whenever necessary. A node (the father) is split by selecting a white edge $v w$, and making two new subproblems or nodes (the children) in the tree, one in which $v w$ is colored red, and one in which $v w$ is colored black. Since, in any chordalization all vertex pairs are colored either red or black, it is clear that every feasible solution of the father node is present in one of the two children. At any stage of the tree, the leaf nodes, the nodes that have not been split, represent the whole set of feasible solutions, guaranteeing that the optimal solution to the root problem can also be found in one of these nodes. The splitting process is called branching. The selection of the edge on which to split, is called branching variable/edge selection.

For some of the leaf nodes, one might be able to conclude that no further examination is necessary. These nodes are labeled inactive, and no further splitting is necessary. The other leaf nodes are called active. Every time, after a node is split, a new active node has to be selected to be processed next. There are several orderings of the active nodes in which to select them. They are described below.

- Breadth First Search: here the subproblems are processed in the order of their creation. This strategy is seldom used.

- Depth First Search: here one always selects the last created subproblem. This is the easiest way of implementing the branching strategy. It also uses the least amount of memory, since the number of subproblems is relatively small.

- Best First Search: here the node is selected according to some criterion, to judge the potential of the node. Generally, in minimization problems the node with the smallest lower bound is selected. Note that both Depth First Search and Breadth First Search can be viewed as special cases of Best First Search using the tree depth within the Branch-and-Bound tree as criterion: taking highest depth and lowest depth as the selection criterion, respectively. 
Before a node is split, it is processed with the techniques available, to find out whether it might still hold the optimal solution to the root problem. A standard way of processing is bounding. In a minimization problem such as determining treewidth, normally a good feasible solution (forming an upper bound) is known, i.e. a good chordalization of input graph $G$ is known, with width val. If one can prove, with some kind of lower bounding technique, that for the subproblem at hand no chordalizations with value smaller than val can be found, then one does not need to examine this node any further; it is made inactive. This is the bounding procedure in the branch-and-bound algorithm. Besides bounding, other methods are used to draw conclusions about the subproblem at hand, such as the addition of red and black edges, and (almost) simplicial vertices. Together these ideas form the processing part in a node.

For all facets of the branch-and-bound technique that are mentioned here, ideas and methods will be described next, some of which are standard, others more problem specific.

\subsection{1 branching rules}

Branching in a node of the binary branch-and-bound tree is done by selecting a white edge in the graph corresponding to the node. Two children of the node at hand are then created by turning the selected white edge into a black edge in one child node respectively into a red edge in the other child node.

\section{simple rules}

The first two branching rules are pretty straightforward in the sense that they select a white edge $e$ based on local properties of the graph in the subproblem at hand, and therefore require only little computation time.

The first rule selects a white edge $v w$ where vertex $v$ has the highest degree (wrt the black edges). $w$ is then selected as the vertex with the highest degree (wrt the black edges) among the vertices for which $v w$ is a white edge. Slightly different mechanisms are possible here: maximum total degree of $v$ and $w$ or including red edges in the selection process.

The second rule is based on extending some clique $C$, in which all edges are colored red or black. It starts off with a maximal clique $C$ in the original graph. One can choose this initial $C$, for instance, by taking a good tree decomposition of $G$, and taking the largest bag. It starts then, hopefully, in the (dense) part of the graph which is the most complicated to chordalize and where it may be easiest to draw conclusions from coloring a white edge red or black. The vertex $w$ outside $C$ is chosen that has the fewest white edges to $C$. Then a white edge $e$ between $w$ and a vertex in $C$ is chosen to branch on. Once all edges between $w$ and $C$ are defined, $C$ can be extended by adding $w$ to $C$. 


\section{probing based rules}

Another way of branching is based on the processing rules that will be discussed in Section 3.5. While turning a white edge of $S$ into a black or red edge, sometimes one can conclude that to obtain a chordalization of the resulting graph, other white edges have to be turned into black edges (or cannot be turned into black edges). By applying these processing rules recursively, the effects of fixing the color of one white edge can be far reaching. For edge $w \in W$, let $w_{b}$ be the number of white edges in $S$ whose color can be fixed after recursively applying the processing rules from Section 3.5 after $w$ is colored black. Similarly, let $w_{r}$ be the number of white edges in $S$ whose color can be fixed after $w$ is colored red. The probing based branching rule considers all white edges $w$ in $S$ and branches on the one for which $w_{b}+w_{r}$ is maximized. This rule greatly reduces the number of subproblems. However, the time won by pruning the subproblems will often be abolished by the time needed to compute $w_{b}+w_{r}$ for all white edges. Variants on this probing based rule are relatively easy to come up with. To save computation time, one might for example apply the processing rules only once instead of recursively.

\subsection{2 bounding methods}

As mentioned before, methods for bounding the treewidth in a node of the tree play a crucial role in our branch-and-bound algorithm. In this section, some novel techniques are presented for bounding the treewidth. They exploit information about the set of red edges in the extension $S=(V, B, R)$ of $G=(V, E)$ that corresponds to a node in the branch-andbound tree.

\section{upper bounding}

To obtain an upper bound on $t w(G)$ that can be used from the very start of a run of the branch-and-bound algorithm, one might apply a quick upper bound heuristic, see Section 1.7.3. In case the results are not satisfying, one might consider to apply a more advanced heuristic like the local search heuristic that is the subject of Chapter 4 of this thesis.

Consider an extension $S=(V, B, R)$ of $G$ and the graph $S^{\prime}$ that has the same set of black edges as $S$ but an empty set of red edges. Any chordalization of $S^{\prime}$ is also a chordalization of $G$. If $S^{\prime}$ happens to be close to an optimal chordalization of $G$, an upper bound heuristic ran on $S^{\prime}$ will likely give a lower upper bound on $t w(G)$ than the same heuristic ran on $G$, simply because there is less room for deviating from an optimal solution. Therefore, in an attempt to improve upon the starting upper bound on $t w(G)$, it might be beneficial to run the upper bound heuristic from time to time on $S^{\prime}$ for some nodes deep in the branch-and-bound tree.

If one can verify that in some node the extension $S=(V, B, R)$ of $G=(V, E)$ is chordal, then obviously its width also provides an upper bound on $t w(G)$. Checking whether $S$ is 
chordal is straightforward and determining the treewidth of a chordal graph can be done in polynomial time, see Lemma 3.2.2.

\section{lower bounding with $R$}

The problem to decide whether an extension $S=(V, B, R)$ of $G=(V, E)$ has a chordalization can be described in the following way: decide whether a chordal graph $S_{C}$ exists such that $S$ is a proper subgraph of $S_{C}$ and $S_{C}$ is a proper subgraph of the graph obtained from $S$ by making all white edges black. This problem is known as the chordal sandwich problem, which was proven to be NP-complete in (65). This destroys hope of effectively killing the subproblems for which no chordalization exist. Nonetheless, some techniques will be introduced here for bounding $t w(S)$ from below. If this lower bound on $t w(S)$ is equal to or larger than a known upper bound on $t w(G)$, the particular node can be set to inactive.

Any chordalization of $S=(V, B, R)$ can be obtained by applying some elimination ordering to $S$. A chordalization of $S$ is an extension of $S$. Hence, at the moment of elimination of vertex $v$, there should not be any red edges between neighbors of $v$. Let $V_{b}$ be the set of vertices $v$ in $S$ for which there are no red edges between neighbors of $v$ in $S$. Based on the foregoing observations, the following lemma provides a lower bound on $t w(S)$ :

Lemma 3.4.1. The minimum degree in $S$ of the vertices from $V_{b}$ is a lower bound on $t w(S)$.

To obtain a chordalization of $S$ by use of an elimination ordering, one of the vertices from $V_{b}$ must be eliminated first. This observation supports the following lemma:

Lemma 3.4.2. Let $C_{b}$ be the vertices in $S$ that are adjacent to all vertices in $V_{b}$. Then $C_{b}$ forms a clique in every chordalization of $S$ and hence $\left|C_{b}\right|-1$ is a lower bound on $t w(S)$.

Next a second lower bound on $t w(S)$ will be described which is also based on the presence of red edges and can be seen as a generalization of a result from (49). Note again that a path in $S$ refers to a path of which all edges are black.

Lemma 3.4.3. Let $S=(V, B, R)$ and let $u v \in R$. Then the number of vertex disjoint paths between $u$ and $v$ in $S$ forms a lower bound on $t w(S)$.

Proof. If $S$ has no chordalization, $t w(S)=\infty$ by definition and the claim is true. Otherwise, consider an arbitrary chordalization of $S$ and a tree decomposition $(T, X)$ of $S$ of equal width that is derived from this chordalization by Algorithm 2.3. Since $u v \in R$, in $(T, X)$ there is no bag that contains both $u$ and $v$. Let $X_{u}$ and $X_{v}$ be the bags in $(T, X)$ that contain respectively $u$ and $v$ such that all internal bags (if any) on the unique path from $X_{u}$ to $X_{v}$ in $T$ contain $u$ nor $v$. Then by the properties of a tree decomposition, bag $X_{u}$ (the same holds for $X_{v}$ ) must contain an internal vertex of any vertex disjoint path between $u$ and $v$ in (the chordalization of) $S$. If there are $w$ such vertex disjoint paths in $S$, then $\left|X_{u}\right| \geq w+1$. The width of $(T, X)$ and of the chordalization of $S$ are thus at least $w$. 
To determine the maximum number of vertex disjoint paths between $u$ and $v$ in $S$, one can use a flow algorithm with capacities on the vertices.

\section{lower bounding with necklaces}

An improvement over the lower bound given by Lemma 3.4.3 can be obtained by using the result of Lemma 3.3.2.

Theorem 3.4.4. Let $P=v_{1} \ldots v_{n}$ be a necklace in $S=(V, B, R)$. Then for any $i \in\{2, \ldots, n-$ $1\}$, the number of vertex disjoint paths between the vertex sets $V_{1}=\left\{v_{1}, \ldots, v_{i-1}\right\}$ and $V_{2}=$ $\left\{v_{i+1}, \ldots, v_{n}\right\}$ forms a lower bound for $t w(S)$.

Proof. If $S$ has no chordalization, $t w(S)=\infty$ by definition and the claim is true. Otherwise, consider an arbitrary chordalization of $S$ and a tree decomposition $(T, X)$ of $S$ of equal width that is derived from this chordalization by Algorithm 2.3. By the definition of a necklace and by Lemma 3.3.2, all paths in the chordalization of $S$ (and thus also in $S$ itself) between a vertex from $V_{1}$ and a vertex from $V_{2}$ have at least one internal vertex. Let $X_{i-1}$ and $X_{i+1}$ be the bags in $(T, X)$ that contain respectively $\left\{v_{i-1}, v_{i}\right\}$ and $\left\{v_{i}, v_{i+1}\right\}$ such that all internal bags (if any) on the unique path from $X_{i-1}$ to $X_{i+1}$ in $T$ do not contain $v_{i-1}$ nor $v_{i+1}$. Note that any internal bag $X_{i}$ on this path contains vertex $v_{i}$ and that $V_{1}$ and $V_{2}$ are in different components of $T \backslash X_{i}$. By the properties of a tree decomposition, bag $X_{i-1}$ (the same holds for $X_{i+1}$ ) must contain an internal vertex of any vertex disjoint path between a vertex from $V_{1}$ and a vertex from $V_{2}$ in (the chordalization of) $S$. If there are $w$ such vertex disjoint paths in $S$, then $\left|X_{i-1}\right| \geq w+1$. The width of $(T, X)$ and of the chordalization of $S$ are thus at least $w$.

Lemma 3.3.2 can also function as a way of bounding the treewidth from below; when a necklace is detected in $S$ and two vertices at distance at least 3 on the necklace are connected by a black edge, then this implies that $t w(S)=\infty$ and the node can be made inactive. The same goes for Lemmas 3.3.3 en 3.3.4.

\section{regular lower bound methods}

Finally consider again the graph $S^{\prime}$ obtained from $S=(V, B, R)$ by deleting all red edges. Since the red edges in $S$ are only restrictive for the number of possible chordalizations of $S$, any lower bound on $t w\left(S^{\prime}\right)$ is also a lower bound on $t w(S)$. By applying regular treewidth lower bound heuristics on $S^{\prime}$ one can thus also bound $t w(S)$ from below. Algorithm 3.1 which was proposed in (64) can therefore be used as a lower bounding procedure in the branch-and-bound method. Independently, the same algorithm was proposed in (33) under the name MMD+(min-d). In the latter paper, two more variants of the algorithm are proposed, called MMD+(max-d) and MMD+(least-c). In step 2a of the algorithm, MMD+(max-d) 
contracts minimum degree vertex $v$ to a maximum degree vertex $u$ in $N(v)$ and algorithm MMD+(least-c) contracts $v$ to a vertex $u$ in $N(v)$ for which $v$ and $u$ have the least number of common neighbors. Experimental results in (33) show that MMD+(least-c) performs slightly better than MMD+(min-d) on several instances. In our implementation MMD+(min-d) is used, for the reason that it takes less computation time.

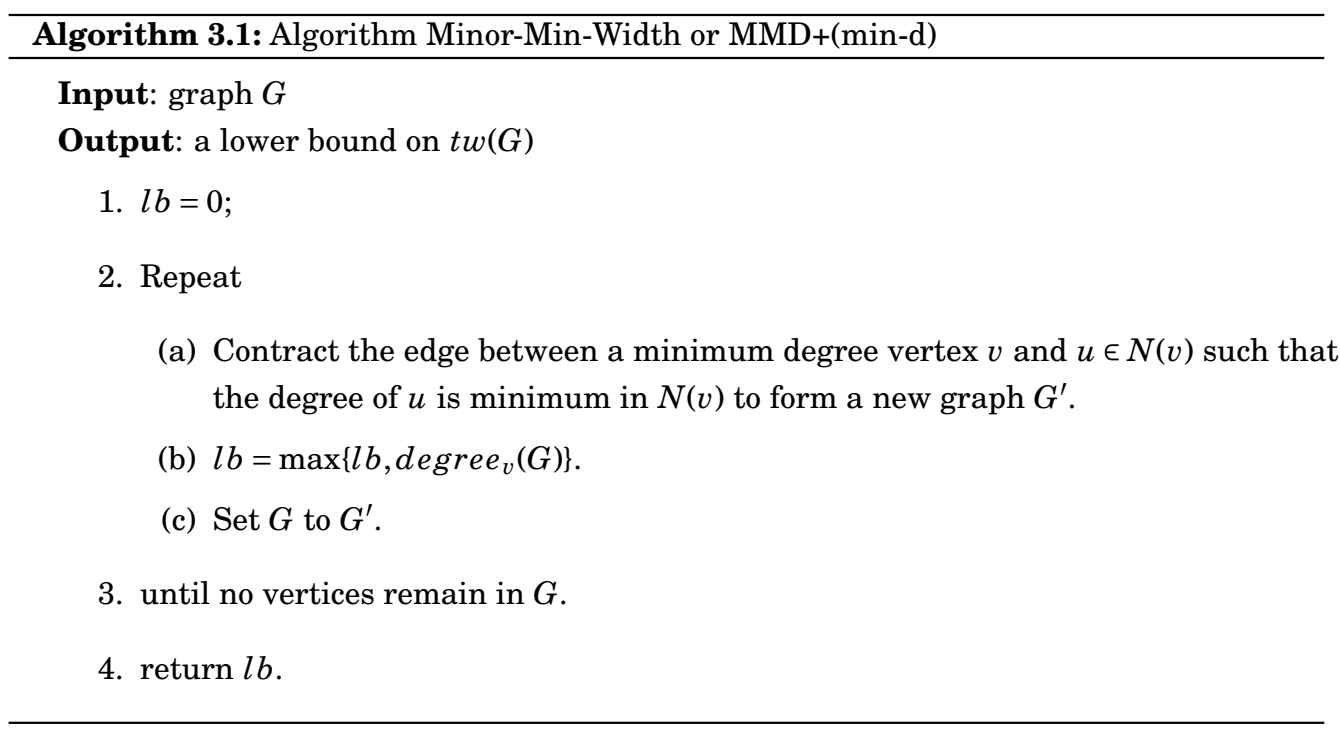

For extensions $S$ of $G$ with only a low number of red edges, Algorithm 3.1 will provide better lower bounds on $t w(S)$ than the $R$-based lower bound algorithms, which exploit information about the set $R$. However, when $|R|$ increases, the $R$-based lower bound methods might be more competitive.

\subsection{Rules for processing}

The problem to determine $t w(S)$ in a node of the branch-and-bound tree becomes easier when more white edges are colored red or black. In this section, several rules are described for processing a graph $S$. The three lemmas from section 3.3 state that because of the presence of necklace structures, in a chordalization of $S$ some white edges in $S$ need to be black or cannot be black. In analogy with these three lemmas, three rules for processing a graph $S$ are introduced below.

According to Lemma 3.3.2, a necklace in $S$ will induce a path in any chordalization of $S$. Hence whenever a necklace is encountered in a subproblem, the branch-and-bound algorithm adds red edges to $S$ between non-adjacent vertices of a necklace in $S$. 
Assume that in graph $S$ a cycle $C=v_{1} \ldots v_{n} v_{1}$ is found in which $P=v_{1} \ldots v_{n-1}$ forms a necklace. Then by Lemma 3.3.3, the algorithm simplifies the subproblem of determining $t w(S)$ in the node by connecting all vertices of $P$ to $v_{n}$ via a black edge.

Assume finally that in graph $S$, a cycle $C=v_{1} \ldots v_{n} w v_{1}$ exists of length at least 4 in which vertex $w$ is connected with red edges to all vertices in $C$ except its neighbors. Then by Lemma 3.3.4, in all chordalizations of $S$, edge $v_{1} v_{n}$ will be black. Hence the algorithm will process the subproblem by coloring the edge black.

The three rules described above together form the set of so called necklace rules.

\subsection{1 simplicial vertices}

Another way of simplifying the subproblem of determining $t w(S)$ is by reducing the size of the graph $S$ itself. In this section, some graph reduction rules are presented, based on simplicial vertices and almost simplicial vertices. The techniques described are an adaption of methods that are used for regular graphs (without set $R$ ), see e.g., (31).

Lemma 3.5.1. Let $v$ be a simplicial vertex in $S=(V, B, R)$ of degree $d(v)$ and let $S^{\prime}=S \backslash\{v\}$. Then $t w(S)=\max \left\{d(v), t w\left(S^{\prime}\right)\right\}$.

Proof. Since $v$ and its neighbors form a clique in $S$ of size $d(v)+1$, it follows that $t w(S) \geq d(v)$. $S^{\prime}$ is a minor of $S$, so by Lemma 2.4.3, $t w(S) \geq t w\left(S^{\prime}\right)$. Combining the two observations, if follows that $t w(S) \geq \max \left\{d(v), t w\left(S^{\prime}\right)\right\}$. To show that $t w(S) \leq \max \left\{d(v), t w\left(S^{\prime}\right)\right\}$, consider an optimal tree decomposition of $S^{\prime}$. Since the neighbors of $v$ form a clique in $S^{\prime}$, there will be a bag containing all neighbors of $v$. By adding a neighbor bag to this bag that contains $v$ and all neighbors of $v$, one obtains a tree decomposition of $S$ of width $\max \left\{d(v), t w\left(S^{\prime}\right)\right\}$. Hence $t w(S) \leq \max \left\{d(v), t w\left(S^{\prime}\right)\right\}$.

The next lemma is the basis of a graph reduction rule with respect to almost simplicial vertices. First let us define contraction in the extended graph $S=(V, B, R)$ of $G$.

Definition 3.5.2. A black edge uv in $S=(V, B, R)$ is said to be contracted to $v$ if for all vertices $w$ for which vw is a white edge:

- $v w$ is added to $B$ if $u w \in B$,

- $v w$ is added to $R$ if $u w \in R$,

and $u$ is deleted from $S$.

For an illustration of this definition, see Figure 3.4. In the following lemma, $R_{v}$ denotes the set of vertices $w$ for which $v w \in R$ in $S$ and $l b$ is some lower bound for $t w(S)$. 


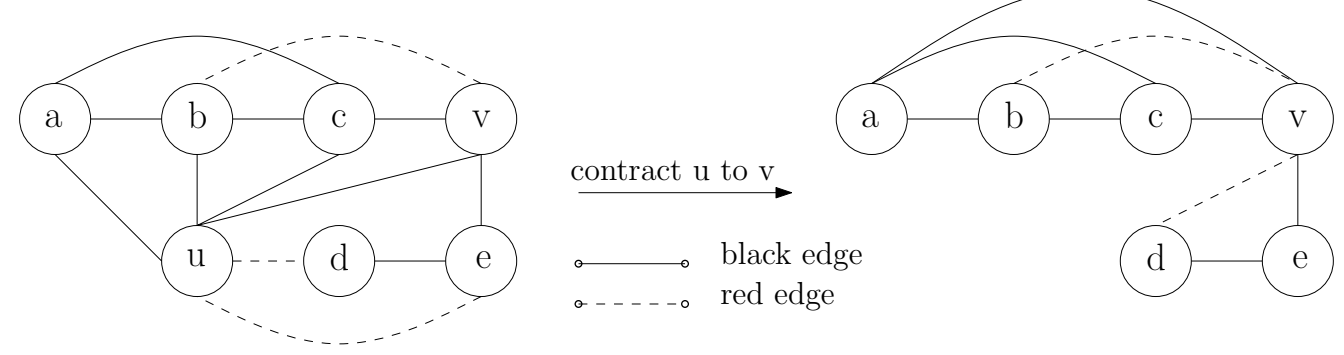

Figure 3.4: contraction of black edge $u v$ to vertex $v$ in graph $S=(V, B, R)$.

Lemma 3.5.3. Let $v$ be an almost simplicial vertex in $S$ with $d(v)<l b$. Let $w$ be the nonclique neighbor of $v$ and let $S^{\prime}$ be the graph that is obtained from $S$ by contracting $v w$ to $w$. If $R_{w} \subseteq R_{v}$, then $t w(S)=t w\left(S^{\prime}\right)$.

Proof. First let us show that $t w(S) \geq t w\left(S^{\prime}\right)$. Consider an optimal tree decomposition of $S$. Replace $v$ by $w$ in the bags of this tree decomposition and delete one $w$ from each bag that contains two $w$ 's. The result is a tree decomposition of $S^{\prime}$ that has lower or equal width. Indeed, the subtree corresponding to $w$ will still be connected, since there was a bag containing both $v$ and $w$ and all edge relations (from $E$ and $F$ ) are still covered by a bag; $w$ will be connected in $S^{\prime}$ to all vertices (except $v$ ) that were either a neighbor of $w$ or $v$ in $S$ and in the new tree decomposition, for each such vertex there is a bag containing $w$ and this vertex. Note that $w$ will form a red edge in $S^{\prime}$ with all vertices that formed a red edge in $S$ with either $v$ or $w$. Since $R_{w} \subseteq R_{v}$ in $S$, neither of those vertices shares a bag with $v$ in the tree decomposition for $S$, and therefore neither of those vertices will share a bag with $w$ in the tree decomposition for $S^{\prime}$. It follows that $t w(S) \geq t w\left(S^{\prime}\right)$.

Now let us show that $t w(S) \leq t w\left(S^{\prime}\right)$. Consider an optimal tree decomposition of $S^{\prime}$. Since $R_{w} \subseteq R_{v}$ in $S$, all neighbors of $v$ in $S$ form a clique in $S^{\prime}$ and therefore there is a bag $X$ in the tree decomposition of $S^{\prime}$ containing all neighbors of $v$ in $S$. Make a bag $Y$ containing vertex $v$ and all its neighbors in $S$ and attach it to bag $X$. The result is a tree decomposition of $S$. Indeed, there is no bag containing both end vertices of a red edge in $S$. The width of this tree decomposition is equal to the maximum of $t w\left(S^{\prime}\right)$ and the degree of vertex $v$ in $S$, i.e., $t w(S) \leq \max \left\{d(v), t w\left(S^{\prime}\right)\right\}$. Since the degree of $v$ in $S$ is smaller than $l b$, the conclusion is that $t w(S) \leq t w\left(S^{\prime}\right)$. 


\subsection{2 conflict graph}

Finally, we propose the idea of keeping track of a so called conflict graph. A conflict graph should be constructed in such a way that it visualizes the conflicts in certain colorings of the white edges of the graph in the subproblem. The conflict graph should have two vertices for each white edge in the graph, one for the case that the edge is colored black and one for the case the edge is colored red. Suppose that $e_{1}$ and $e_{2}$ are potential edges in the input graph, then the conflict graph has vertices $c_{1 b}, c_{1 r}, c_{2 b}$ and $c_{2 r}$. Vertex $c_{1 b}$ corresponds with the situation where $e_{1}$ is a black edge and $c_{1 r}$ to the situation where $e_{1}$ is a red edge. Suppose that as a result of the necklace lemmas, $e_{1}$ can not be colored black if $e_{2}$ is red, then the conflict graph has an edge $c_{1 b} c_{2 r}$. Conflicts that are detected by the processing rules in a node of the branch-and-bound tree remain valid for the children of this node. By apply the processing rules again in the child node, one has to determine the same conflicts in the child node all over again. The advantage of keeping track of a conflict graph is that from a node to its child node, the conflict graph has to be updated only in the local region where the branch-and-bound algorithm is operating at that moment. For this reason we believe that keeping track of a conflict graph would speed up the branch-and-bound algorithm.

\subsection{Experimental results}

The branch-and-bound algorithm has been tested on a number of graph classes. Implementation of the algorithm was done in $\mathrm{C}++$ and experiments were run on a $2.4 \mathrm{GHz}$ Intel Core 2 CPU having 2 GB of RAM. The computational experiments focussed on grid graphs, queen graphs, and graphs derived with the procedure used for Mycielski graphs. These graphs can easily be extended to larger graphs with similar properties. In this way the behavior of the algorithm has be studied on similar instances of increasing size.

For all results in this section, Depth First Search has been used as the traversal strategy in the branch-and-bound tree. Furthermore, branching in the tree was done by the first branching rule as described in Section 3.4.1. Lower bounds were obtained by Algorithm 3.1. Upper bounds were found by checking for chordality in every subproblem. For some graph classes, initial upper bounds were passed as input to the algorithm. Finally, the graphs corresponding to the subproblems were processed according to the rules that are described in Section 3.5. At the moment of writing this chapter, the remaining ideas from this chapter have not yet been fully implemented. For this reason, no running times are displayed in the tables. Instead, the number of nodes visited in the branch-and-bound tree has been used as a performance criterion for our branch-and-bound algorithm.

The tables in this section use the following terminology. $|V|$ and $|E|$ denote respectively the number of vertices and edges in the graph, whereas $u b$ and $t w$ denote an upper bound 
on the treewidth respectively the treewidth of the graph. Finally, columns \#1, \#2, and \#3 display the number of processed subproblems for several variants of the algorithm:

- \#1 denotes the number of processed subproblems when the algorithm is used in its most basic form; except for removal of simplicial vertices (which is needed to determine whether the graph is chordal), no processing is used,

- \#2 denotes the number of processed subproblems when the necklace rules for processing are enabled,

- \#3 denotes the number of processed subproblems when in addition to the necklace rules also the (almost) simplicial vertices rules are enabled.

\subsubsection{Grid graphs}

For $n, m \geq 2$, the $(n \times m)$-grid graph (see (96)) is the simple graph with vertices $v_{i j}(1 \leq i \leq$ $n, 1 \leq j \leq m)$ where $v_{i j}$ and $v_{i^{\prime} j^{\prime}}$ are adjacent if $\left|i-i^{\prime}\right|+\left|j-j^{\prime}\right|=1$. The $(n \times m)$-grid graph will be denoted in this section as grid $n \_m$. Grid graphs are relatively sparse and hence the graph corresponding to the root problem has a lot of white edges. This makes it relatively difficult for the branch-and-bound algorithm to find initial chordalizations that can be used as an upper bound. The treewidth of grid $n \_m$, known to be $\min (n, m)$, is therefore passed as an upper bound to the algorithm. Experimental results of the branch-and-bound algorithm on grid graphs can be found in Table 3.1.

\begin{tabular}{|l|c|c|c|c|r|r|r|}
\hline graph & $|V|$ & $|E|$ & ub & tw & $\# 1$ & $\# 2$ & $\# 3$ \\
\hline \hline grid 3_3 & 9 & 12 & 3 & 3 & 401 & 39 & 39 \\
grid 3_4 & 12 & 17 & 3 & 3 & 1835 & 67 & 67 \\
grid 3_5 & 15 & 22 & 3 & 3 & 6109 & 105 & 105 \\
grid 3_6 & 18 & 27 & 3 & 3 & 35455 & 293 & 293 \\
grid 3_7 & 21 & 32 & 3 & 3 & 171711 & 743 & 743 \\
grid 3_8 & 24 & 37 & 3 & 3 & 929393 & 2019 & 2019 \\
grid 3_9 & 27 & 42 & 3 & 3 & 4754479 & 5501 & 5501 \\
grid 3_10 & 30 & 47 & 3 & 3 & $>10^{7}$ & 15001 & 15001 \\
grid 4_4 & 16 & 24 & 4 & 4 & $>10^{7}$ & 638739 & 298325 \\
grid 4_5 & 20 & 31 & 4 & 4 & $>10^{7}$ & 9652057 & 4821991 \\
\hline
\end{tabular}

Table 3.1: Experiments of the algorithm on Grid graphs 


\subsubsection{Queen graphs}

The $n$ by $m$ queen graph, denoted by queen $n \_m$, is a graph that is based on a chess board of $n$ by $m$ squares. Each square corresponds to one vertex in the graph. Vertices $v$ and $w$ are connected by edges if a queen is allowed to move from the square corresponding to $v$ to the square corresponding to $w$, i.e. one or more positions horizontally, vertically, or diagonally on the board.

While testing the branch-and-bound algorithm on the queen graphs, no initial upper bound was used. Experimental results of the algorithm on queen graphs can be found in Table 3.2. The (small instances of) queen graphs are dense and hence the graphs corresponding to the subproblems have relatively few white edges. This makes processing rules more effective, but at the same time the high treewidth of the queen graphs makes finding an optimal chordalization hard.

\begin{tabular}{|l|c|c|c|r|r|r|}
\hline graph & $|V|$ & $|E|$ & tw & $\# 1$ & $\# 2$ & $\# 3$ \\
\hline \hline queen 3_3 & 9 & 28 & 6 & 5 & 5 & 5 \\
queen 3_4 & 12 & 46 & 8 & 381 & 59 & 49 \\
queen 3_5 & 15 & 67 & 10 & 20823 & 561 & 433 \\
queen 3_6 & 18 & 91 & 12 & 1484735 & 11589 & 9383 \\
queen 3_7 & 21 & 118 & 14 & $>10^{7}$ & 472621 & 392947 \\
queen 4_4 & 16 & 76 & 11 & 37909 & 767 & 551 \\
queen 4_5 & 20 & 110 & 14 & $>10^{7}$ & 93487 & 69055 \\
queen 4_6 & 24 & 148 & 16 & $>10^{7}$ & 1301913 & 1159711 \\
\hline
\end{tabular}

Table 3.2: Experiments of the algorithm on Queen graphs

\subsubsection{Mycielski like graphs}

The algorithm was tested on Mycielski extensions of paths $P_{n}$, cycles $C_{n}$, and cliques $K_{n}$ of increasing size. The Mycielski extension of a graph can be found by adding copies of all vertices, connecting them to the neighbors of their original and finally adding one extra vertex that is connected to all the copies. Results can be found in Tables 3.3, 3.4 and 3.5. The subscripts $m 1$ and $m 2$ in these tables mean that respectively 1 and 2 rounds of the Mycielski construction were applied to the cycles, paths or cliques. Upper bounds that were obtained from a heuristic were passed as input to the algorithm. 


\begin{tabular}{|l|c|c|c|c|c|}
\hline graph & $|V|$ & $|E|$ & $\mathrm{ub}$ & $\mathrm{tw}$ & $\# 3$ \\
\hline \hline$C_{3 \_\mathrm{m} 1}$ & 7 & 12 & 3 & 3 & 1 \\
\hline$C_{4 \_} \mathrm{m} 1$ & 9 & 16 & 4 & 4 & 23 \\
\hline$C_{5 \_} \mathrm{m} 1$ & 11 & 20 & 5 & 5 & 259 \\
\hline$C_{6 \_} \mathrm{m} 1$ & 13 & 24 & 5 & 5 & 1457 \\
\hline$C_{7 \_} \mathrm{m} 1$ & 15 & 28 & 5 & 5 & 11537 \\
\hline$C_{8 \_\mathrm{m} 1}$ & 17 & 32 & 5 & 5 & 100317 \\
\hline$C_{9 \_\mathrm{m} 1}$ & 19 & 36 & 5 & 5 & 854125 \\
\hline$C_{10 \_\mathrm{m} 1}$ & 21 & 40 & 5 & 5 & 7627301 \\
\hline$C_{3 \_\mathrm{m} 2}$ & 15 & 43 & 7 & 7 & 727 \\
\hline$C_{4 \_} \mathrm{m} 2$ & 19 & 57 & 8 & 8 & 17823 \\
\hline$C_{5 \_} \mathrm{m} 2$ & 23 & 71 & 11 & 10 & $>10^{7}$ \\
\hline$C_{6 \_} \mathrm{m} 2$ & 27 & 85 & 11 & 10 & $>10^{7}$ \\
\hline
\end{tabular}

Table 3.3: Experiments of the algorithm on Mycielski-cycles

\begin{tabular}{|l|c|c|c|c|c|}
\hline graph & $|V|$ & $|E|$ & ub & tw & $\# 3$ \\
\hline \hline$P_{3 \_m 1}$ & 7 & 9 & 2 & 2 & 1 \\
\hline$P_{4 \_} 1$ & 9 & 13 & 3 & 3 & 9 \\
\hline$P_{5 \_m 1}$ & 11 & 17 & 3 & 3 & 9 \\
\hline$P_{6 \_\mathrm{m} 1}$ & 13 & 21 & 3 & 3 & 9 \\
\hline$P_{7 \_\mathrm{m} 1}$ & 15 & 25 & 3 & 3 & 9 \\
\hline$P_{\text {8_m1 } 1}$ & 17 & 29 & 3 & 3 & 9 \\
\hline$P_{9 \_\mathrm{m} 1}$ & 19 & 33 & 3 & 3 & 9 \\
\hline$P_{10 \_\mathrm{m} 1}$ & 21 & 37 & 3 & 3 & 9 \\
\hline$P_{3 \_\mathrm{m} 2}$ & 15 & 34 & 6 & 5 & 55 \\
\hline$P_{4 \_\mathrm{m} 2}$ & 19 & 48 & 7 & 7 & 640091 \\
\hline$P_{5 \_\mathrm{m} 2}$ & 23 & 62 & 8 & 7 & 530707 \\
\hline$P_{6 \_\mathrm{m} 2}$ & 27 & 76 & 8 & 8 & $>10^{7}$ \\
\hline
\end{tabular}

Table 3.4: Experiments of the algorithm on Mycielski-paths

\subsection{Conclusions}

In this chapter, an exact (branch-and-bound) algorithm is described for determining the treewidth of a graph $G=(V, E)$. The algorithm constructs all chordalizations $H=(V, E \cup F)$ of $G$ (explicitly or implicitly) by splitting the solution space on the basis of adding/forbidding edges in the chordalization. This in contrast to other exact algorithms that use a solution space based on e.g. elimination orderings to determine the treewidth. 


\begin{tabular}{|l|c|c|c|c|c|}
\hline graph & $|V|$ & $|E|$ & ub & tw & $\# 3$ \\
\hline \hline$K_{4 \_\mathrm{m} 1}$ & 9 & 22 & 4 & 4 & 1 \\
\hline$K_{5 \_} 1$ & 11 & 35 & 5 & 5 & 1 \\
\hline$K_{6 \_\mathrm{m} 1}$ & 13 & 51 & 6 & 6 & 1 \\
\hline$K_{7 \_\mathrm{m} 1}$ & 15 & 70 & 7 & 7 & 1 \\
\hline$K_{8 \_\mathrm{m} 1}$ & 17 & 92 & 8 & 8 & 1 \\
\hline$K_{9 \_\mathrm{m} 1}$ & 19 & 117 & 9 & 9 & 1 \\
\hline$K_{10 \_} 1$ & 21 & 145 & 10 & 10 & 1 \\
\hline$K_{4 \_\mathrm{m} 2}$ & 19 & 75 & 9 & 9 & 1831 \\
\hline$K_{5 \_\mathrm{m} 2}$ & 23 & 116 & 11 & 11 & 6033 \\
\hline$K_{6 \_\mathrm{m} 2}$ & 27 & 166 & 13 & 13 & 20553 \\
\hline$K_{7 \_\mathrm{m} 2}$ & 31 & 225 & 15 & 15 & 168981 \\
\hline$K_{8 \_\mathrm{m} 2}$ & 35 & 293 & 17 & 17 & 942903 \\
\hline$K_{9 \_\mathrm{m} 2}$ & 39 & 370 & 19 & 19 & $>10^{7}$ \\
\hline$K_{4 \_\mathrm{m} 3}$ & 39 & 244 & 18 & 18 & $>10^{7}$ \\
\hline
\end{tabular}

Table 3.5: Experiments of the algorithm on Mycielski-cliques

The current version of the algorithm uses a selection of the ideas that are put forward in this chapter and has been tested on a number of graph classes. The results show that the algorithm in its current state can solve graph instances up to some 30 vertices, if the treewidth is not too high. The processing rules as far as they are implemented now, significantly reduce the number of subproblems that need to be processed. However, the results are not yet comparable with the branch-and-bound methods of Gogage and Dechter (64) and Bachoore and Bodlaender (10). Some additional ideas have been discussed in this chapter. Section 3.4.2 describes several lower bound procedures that can be incorporated. These might be improvements over the current, more simple lower bounding procedure. In Section 3.5.2 the idea is proposed to keep track of a conflict graph. We do have confidence in the quality of these ideas, but we realize that the proof of concept still has to be done. 



\section{Chapter 4}

\section{Local Search}

In the search for tree decompositions of large graph instances, the sheer complexity of the treewidth problem forces one to resort to non-exact methods. The spectrum of such nonexact, constructive treewidth algorithms shows a trade-off between quality of the solution (width of the returned tree decomposition) and computation time that is needed. For large graphs, it often happens that neither the popular upper bound heuristics nor the approximation algorithms are able to find a good balance between these two measures. The first type may return solutions that are exponentially far from optimal and they may do so in far less time than might be available. On the other hand, the second type may take unreasonable amounts of time to find a solution that approximates an optimal solution.

In this chapter, we introduce an upper bound heuristic for treewidth that partly fills this gap. More specifically, we construct a local search heuristic for treewidth that starts with a solution obtained by one of the quick upper bound heuristics and subsequently tries to improve upon this solution by performing a neighborhood search.

The heuristic exploits a new neighborhood structure that operates directly on a tree decomposition of the input graph. This in contrast to earlier local search heuristics for treewidth that generally used derived notions such as elimination orders of the vertices, see for example (50). As a side result, we find an alternative proof for a claim from (17) that chordalizations of a graph can be made minimal with respect to fill-in edges in $\mathrm{O}(f(e+f))$ time, where $f$ denotes the number of fill-in edges in a chordalization.

In Section 4.1, some yet undefined notions and preliminaries will be introduced. Section 4.2 describes in detail the two main building blocks of the neighborhood structure in our local search heuristic. Section 4.3 treats the local search heuristic itself, together with a way to evaluate different solutions and some ideas for restricting the size of the neighborhood. Finally, in Section 4.4, experimental results of an implementation of the heuristic are presented.

The content of this chapter is based on cooperation with Stan van Hoesel and Arie Koster. 


\subsection{Preliminaries}

Definition 4.1.1. A pair of vertices $\{u, v\} \subset V$ is called a fill-in pair in a tree decomposition $(T, X)$ of $G=(V, E)$ if $u v \notin E$ and $\exists X_{i} \in X$ with $u \in X_{i}$ and $v \in X_{i}$. The set of fill-in pairs of $(T, X)$ will be denoted by $F$.

Note that in Chapter 3 the letter $F$ was used for the set of fill-in edges in a chordalization of a graph $G$. We like to point out here that the set $F$ of fill-in pairs in a tree decomposition $(T, X)$ equals the set $F$ of fill-in edges in the chordalization of $G$ that can be obtained from $(T, X)$ using Algorithm 2.2. Given a tree decomposition $(T, X)$, we denote by $F_{i}$ the set of fill-in pairs that are present in exactly $i$ bags of $(T, X)$. Thus for a tree decomposition on $l$ bags, the corresponding set $F$ can be partitioned into $F_{1}, \ldots, F_{l}$. Later in this chapter we will show that elements from $F_{1}$ can simply be 'removed' from $(T, X)$ without increasing its width. For this reason, we call $f \in F_{1}$ a redundant fill-in pair.

Definition 4.1.2. A tree decomposition $(T, X)$ is fill-in-pair minimal if it contains no redundant fill-in pairs, i.e. if $F_{1}=\varnothing$.

A bag $X_{A}$ of a tree decomposition is included in bag $X_{B}$ (or $X_{A} \subseteq X_{B}$ ) if all vertices of $X_{A}$ are contained in $X_{B}$.

Definition 4.1.3. A tree decomposition $(T, X)$ is inclusion minimal if no bag of $(T, X)$ is included in another bag of $(T, X)$.

Note that for an inclusion minimal tree decomposition it holds that for any two neighbor bags $X_{A}$ and $X_{B}, X_{A}$ contains a vertex that is not present in $X_{B}$, and vice versa. The inclusion minimality property can easily be obtained by contracting bags to bags which they are included in.

Definition 4.1.4. A tree decomposition $(T, X)$ is minimal if it is both inclusion minimal and fill-in-pair minimal.

Given a tree decomposition $(T, X)$ of $G=(V, E)$ and $S \subset V$, by $T_{S}$ we denote the subgraph of $T$ induced by the bags that contain all vertices in $S$. By the properties of a tree decomposition, $T_{S}$ is always a subtree of $T$. Indeed, if two bags $X_{A}$ and $X_{B}$ of $(T, X)$ contain all vertices from $S$, then the bags on the unique path in $T$ between $X_{A}$ and $X_{B}$ do so as well.

\subsection{Neighborhood structure}

In this section, we define two basic operations on a tree decomposition that we will call merge and split. The merge of two neighbor bags $X_{A}$ and $X_{B}$ in tree $T$ is a contraction of 
bags $X_{A}$ and $X_{B}$ into a new bag $X_{C}$, where $X_{C}$ contains the union of the vertices of $X_{A}$ and $X_{B}$. All bags (except $X_{A}$ and $X_{B}$ themselves) that were neighbor to either $X_{A}$ or $X_{B}$ before the merge are neighbor to $X_{C}$ after merging $X_{A}$ and $X_{B}$. Note that the validity of a tree decomposition is invariant under a merge operation: the three properties of a tree decomposition are not violated by a merge. A subtree $T_{S}$ is said to be merged in $(T, X)$ if the bags of $T_{S}$ are pairwise merged until $T_{S}$ is a single bag in $(T, X)$. Though a merge operation may create redundant fill-in pairs (as we will see later), it cannot destroy inclusion minimality of a tree decomposition.

A split of a bag is in some sense the opposite operation of a merge. During a split, one bag $X_{C}$ in $(T, X)$ is replaced by two new neighboring bags $X_{A}$ and $X_{B}$, such that the union of the vertex sets of $X_{A}$ and $X_{B}$ is equal to the vertex set of $X_{C}$ and $\left|X_{A}\right|=\left|X_{B}\right|=\left|X_{C}\right|-1$. When bag $X_{C}$ is split into the bags $X_{A}$ and $X_{B}$, there will be a vertex pair $\{u, v\}$ in $X_{C}$ that is present in neither $X_{A}$ nor $X_{B}$. We say that such a vertex pair is separated during the split. Assume w.l.o.g. that bag $X_{A}$ contains vertex $u$ and bag $X_{B}$ contains $v$. Now suppose that before the split, vertex pair $\{u, v\}$ was not only present in bag $X_{C}$, but also in some bag $X_{D}$. Then in order to maintain connectivity of $T_{u}$ and $T_{v}$, in the tree decomposition after the split there must be a path from $X_{D}$ to $X_{A}$ not containing $X_{B}$ and a path from $X_{D}$ to $X_{B}$ not containing $X_{A}$. Because $X_{A}$ and $X_{B}$ are neighbors in the new tree, this would imply that $T$ contains a cycle, a contradiction. Hence a vertex pair $\{u, v\}$ in bag $X_{C}$ can only be separated by the split operation if it is exclusively present in $X_{C}$. In addition, $\{u, v\}$ can only be separated when it is a fill-in pair, i.e., when it does not correspond to an element in $E$, since else the resulting tree decomposition is not valid. A bag $X_{C}$ can thus only be split into neighbor bags $X_{A}$ and $X_{B}$ when $X_{C}$ contains a redundant fill-in pair, i.e. an element from $F_{1}$. A split operation thus essentially removes a redundant fill-in pair from the tree decomposition.

Definition 4.2.1. Given a bag $X_{C}$ in the tree decomposition $(T, X)$ and $\{u, v\} \in F_{1}$ in $X_{C}$, we say that $\{u, v\}$ is removed from $(T, X)$ when:

1. $X_{C}$ is split into neighbor bags $X_{A}=X_{C} \backslash\{u\}$ and $X_{B}=X_{C} \backslash\{v\}$ and

2. The former neighbor bags of $X_{C}$ that contain vertex $v$ are reconnected to $X_{A}$ and the other former neighbor bags of $X_{C}$ to $X_{B}$.

Note that in step 2 neighbor bags of $X_{C}$ containing neither $v$ nor $u$ can either be connected to $X_{A}$ or to $X_{B}$. It is not difficult to check that validity of the tree decomposition is maintained under removal of a redundant fill-in pair. Removing a redundant fill-in pair does not necessarily maintain the inclusion minimality property of a tree decomposition. It can be shown however that removing a redundant fill-in pair does not create any new redundant fill-in pairs. When removing a redundant fill-in pair $\{u, v\}$ by splitting bag $X_{C}$, 
other redundant fill-in pairs in $X_{C}$ that are incident to neither $u$ nor $v$ will be present both in bag $X_{A}$ and $X_{B}$ afterwards, i.e. they move from $F_{1}$ to $F_{2}$. For an example of operations merge and split, see Figure 4.1. This figure illustrates that a merge operation can create new redundant fill-in pairs and that a split operation can result in one bag being included in another bag.

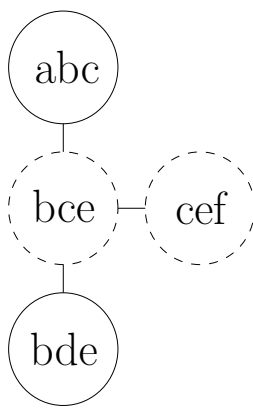

(a)

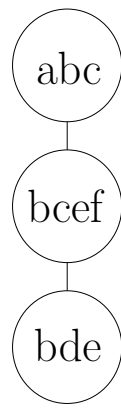

(b)

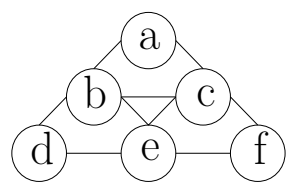

(c)

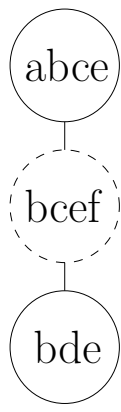

(d)

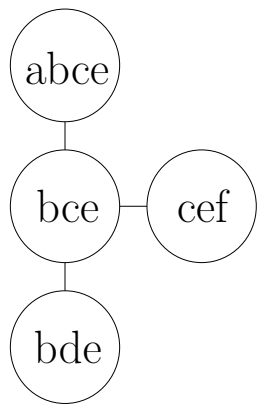

(e)

Figure 4.1: Picture (a) shows a fill-in pair minimal tree decomposition of the graph in (c). By merging the two dashed bags, pair $\{b, f\}$ becomes redundant, see (b). Picture (d) shows an inclusion minimal tree decomposition of the graph in (c). When removing redundant fill-in pair $\{b, f\}$ by splitting the dashed bag, the inclusion minimality property will be lost, see (e).

The neighborhood structure that will be employed in the local search heuristic can be described in terms of two procedures. The first one removes a fill-in pair $\{u, v\}$ from some initial minimal tree decomposition. This is done by merging $T_{\{u, v\}}$ into a single bag such that $\{u, v\}$ becomes a redundant fill-in pair and subsequently removing $\{u, v\}$ by splitting the merged bag. During the second procedure, minimality of the resulting tree decomposition is reattained. We construct an $O(f(e+f))$ algorithm that, given a tree decomposition with fill-in pair set $F$ and width $k$, returns a minimal tree decomposition with fill-in pair set $F^{\prime} \subseteq F$ and width $k^{\prime} \leq k$. The neighborhood of a minimal tree decomposition $(T, X)$ can now be defined by the set of all minimal tree decompositions that can be obtained from $(T, X)$ by sequentially applying the two procedures. In the following subsections, the two procedures will be explained in more detail.

\subsection{1 procedure 1: removing a fill-in pair}

An important observation is that for any pair $\{u, v\} \notin E$, there exists a tree decomposition $(T, X)$ of $G=(V, E)$ such that $\{u, v\} \notin F$. Given $(T, X)$ with $\{u, v\} \in F$, the following algorithm shows how to remove an arbitrary fill-in pair $\{u, v\}$ from $F$ using merge and split operations 
as they are defined in the previous section.

Algorithm 4.1: remove a fill-in pair from $(T, X)$
Input: Tree decomposition $(T, X)$ with $\{u, v\} \in F$
Output: Tree decomposition $(T, X)$ with $\{u, v\} \notin F$
1. Merge $T_{\{u, v\}}$ into a single bag to make $\{u, v\}$ redundant.
2. Remove redundant fill-in pair $\{u, v\}$.

It is easy to see that inclusion minimality is maintained under the merge step in Algorithm 4.1. Also, it is not difficult to verify that a tree decomposition remains valid under application of Algorithm 4.1. Removing the redundant fill-in pair in the second step of Algorithm 4.1 is done by application of the steps in Definition 4.2.1. Note that when Algorithm 4.1 is applied to a redundant fill-in pair, the first step can be skipped. When Algorithm 4.1 is applied to remove fill-in pair $\{u, v\}$, we will from now on refer to the single bag that $T_{\{u, v\}}$ is merged into as bag $X_{M}$ and to the bags $X_{M} \backslash\{u\}$ and $X_{M} \backslash\{v\}$ after step 2 as respectively $X_{M_{v}}$ and $X_{M_{u}}$. Figure 4.2 provides an illustration of Algorithm 4.1.

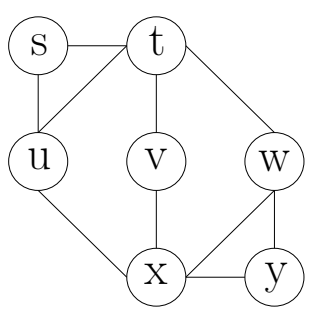

(a)

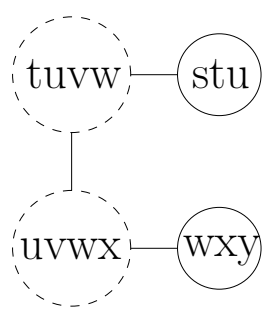

(b)

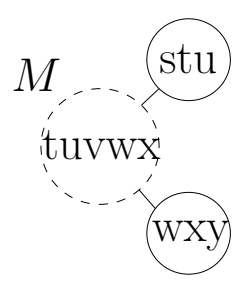

(c)

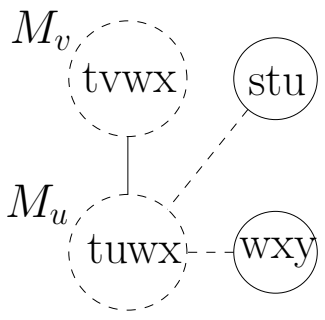

(d)

Figure 4.2: Picture (b) shows a tree decomposition of the graph in (a) with $\{u, v\} \in F_{2}$. In (c), subtree $T_{\{u, v\}}$ is merged into a single bag $X_{M}$ and hence $\{u, v\}$ moves to $F_{1}$. Subsequently in (d), redundant fill-in pair $\{u, v\}$ is removed by splitting $X_{M}$ into neighbor bags $X_{M_{u}}$ and $X_{M_{v}}$. The two neighbor bags of $X_{M}$ in (c) do not contain vertex $v$ so both of them are connected to $X_{M_{u}}$.

In the second procedure that will be described in the next section, all elements from $F_{1}$ will be removed in order to regain minimality of the tree decomposition. It is therefore important to know what happens to the set $F_{1}$ during the first procedure. The following two lemmas specify for each of the two steps in Algorithm 4.1 what happens to the set of redundant fill-in pairs $F_{1}$. 
Lemma 4.2.2. Let $(T, X)$ be a tree decomposition of a graph $G$ with fill-in pair $\{u, v\}$. When $T_{\{u, v\}}$ is merged into a single bag $X_{M}$, no elements disappear from $F_{1}$ and only the following two types of fill-in pairs will move to $F_{1}$ :

(1) Fill-in pairs $\{i, j\}$ for which $\left|T_{\{i, j\}}\right| \geq 2$ and $T_{\{i, j\}}$ is a subtree of $T_{\{u, v\}}$.

(2) Pairs $\{i, j\}$ for which $T_{\{i, j\}}=\varnothing$ and both $T_{i}$ and $T_{j}$ intersect with $T_{\{u, v\}}$.

Proof. $T_{\{u, v\}}$ is merged into $X_{M}$ by repeatedly merging pairs of neighbor bags in $T_{\{u, v\}}$. Any redundant fill-in pair that was present in one of the bags of $T_{\{u, v\}}$ will afterwards be present only in $X_{M}$, so it will still be redundant. Now consider a redundant fill-in pair $\{a, b\}$ from a bag not in $T_{\{u, v\}}$. Then at least one of the trees $T_{a}$ or $T_{b}$ does not intersect with $T_{\{u, v\}}$. Indeed if they did, then the bag $T_{\{a, b\}}$ should also intersect with $T_{\{u, v\}}$, a contradiction. Thus fill-in pair $\{a, b\}$ will not be present in $X_{M}$ and hence it will remain redundant after the merge. While merging $T_{\{u, v\}}$ into $X_{M}$, all redundant fill-in pairs will thus remain redundant. Any fill-in pair that was present in at least 2 bags of $T_{\{u, v\}}$ and in no bag outside $T_{\{u, v\}}$ will only be present in $X_{M}$ after the merge, so it moves from $F_{i}$ (for some $i \geq 2$ ) to $F_{1}$. Finally, consider two vertices that are present in $T_{\{u, v\}}$, but not together in one bag. After merging $T_{\{u, v\}}$ into $X_{M}$, they form a fill-in pair that is present only in $X_{M}$, so they are redundant.

Lemma 4.2.3. While removing $\{u, v\}$ from $(T, X)$ according to Definition 4.2.1, element $\{u, v\}$ drops out of $F_{1}$ and all redundant fill-in pairs that are not incident to $u$ nor $v$ and have the same subtree as $\{u, v\}$ will move from $F_{1}$ to $F_{2}$. All other redundant fill-in pairs remain redundant.

Proof. Let $T_{\{u, v\}}=M$ before removing redundant fill-in pair $\{u, v\}$. First we show that while splitting $X_{M}$ in $X_{M_{u}}$ and $X_{M_{v}}$, no new redundant fill-in pairs are created. Each fill-in pair from $X_{M}$ (except $\{u, v\}$ ) is present in at least one of the bags $X_{M_{u}}$ or $X_{M_{v}}$ after the split, so no fill-in pairs move from $F_{i}$ (for some $i \geq 2$ ) to $F_{1}$. Furthermore, while splitting $X_{M}$, no new fill-in pairs are created, i.e. any pair in $X_{M_{u}}$ or $X_{M_{v}}$ was already present in bag $X_{M}$. Now consider a redundant fill-in pair in a bag other than $X_{M}$ before the split. After splitting $X_{M}$, this pair won't be present in $X_{M_{u}}$ or $X_{M_{v}}$ either, so it remains redundant. Clearly, $\{u, v\}$ itself drops out of $F_{1}$ since it was only present in $X_{M}$ and it is not present in $X_{M_{u}}$ nor $X_{M_{v}}$. Finally consider the other elements of $F_{1}$ from $X_{M}$. If they are incident to $u(v)$, then after the split they are only present in $X_{M_{u}}\left(X_{M_{v}}\right)$, so they remain redundant. If they are not incident to $u$ nor to $v$, then after the split they appear in both $X_{M_{u}}$ and $X_{M_{v}}$, so they move from $F_{1}$ to $F_{2}$.

Before proceeding with the second procedure of regaining minimality of the tree decomposition, we point out that application of the first procedure to a fill-in pair $\{u, v\}$ in a minimal tree decomposition will always lead to a tree decomposition of higher or at best 
equal width. Indeed, $T_{\{u, v\}}$ consists of at least two bags. Given two bags from $T_{\{u, v\}}$, by the inclusion minimality property of $(T, X)$ one of them contains a vertex that is not present in the other one. Thus by merging $T_{\{u, v\}}$ into a single bag $X_{M}$, bag $X_{M}$ will be strictly larger than the largest bag in $T_{\{u, v\}}$. When $X_{M}$ is subsequently split into bag $X_{M_{u}}$ and $X_{M_{v}}$ of size $\left|X_{M}\right|-1$, the size of these two bags is still equal to or larger than the largest bag of $T_{\{u, v\}}$.

The aim of our local search heuristic is obviously to find tree decomposition that have a lower width than some minimal starting solution. The contribution of the first procedure can thus not be found in the width of the resulting tree decomposition. However, as can be concluded from Lemmas 4.2.2 and 4.2.3, Algorithm 4.1 is capable of turning a minimal tree decomposition into a non-minimal one by creating redundant fill-in pairs in the first step. In Figure 4.2 the algorithm turns the minimal tree decomposition in (b) into the nonminimal tree decomposition in (d), where $\{u, w\},\{v, w\} \in F_{1}$. This property will be exploited by the second procedure, which basically removes the newly created redundant fill-in pairs, until minimality is obtained. Clearly, this can potentially decrease the width of the tree decomposition. Whenever the increase in width caused by the first procedure is smaller than the decrease in width caused by the second procedure, the overall result is positive and a better tree decomposition is detected in the neighborhood.

\subsection{2 procedure 2: regaining minimality}

In the previous section it was shown that tree decompositions obtained from a minimal tree decomposition by applying Algorithm 4.1 are likely to lack fill-in-pair minimality. In this section we present the procedure to regain minimality. The procedure is an algorithm called MTDA (Minimal Tree Decomposition Algorithm) that regains minimality by removing redundant fill-in pairs. It takes as input a graph $G$ and an arbitrary tree decomposition $(T, X)$ of $G$ and it returns a minimal tree decomposition of lower or equal width. Moreover, it will not create any new fill-in pairs, so the set of fill-in pairs in the resulting minimal tree decomposition is a subset of the set of fill-in pairs in the input tree decomposition. The complexity of MTDA will be shown to be equal to $O(f(e+f))$, where $f=|F|$ and $e=|E|$.

\section{inclusion minimality}

Minimality of a tree decomposition is defined in terms of inclusion minimality and fill-inpair minimality. We will assume that the input tree decomposition is already inclusion minimal. If this is not the case, we can easily obtain inclusion minimality by the following algorithm. Observe that if in a tree decomposition $(T, X)$ of $G$ bag $X_{A} \subseteq X_{C}$, then bag $X_{A} \subseteq X_{B}$, for all bags $X_{B}$ on the unique path in $T$ from $X_{A}$ to $X_{C}$. Thus, if a bag in $(T, X)$ is not included in any of its neighbor bags, then it will not be included in any other bag of $(T, X)$ either. 
Definition 4.2.4. If bag $X_{A}$ is included in neighbor bag $X_{B}$, we say that we contract $X_{A}$ to $X_{B}$ if we delete $X_{A}$ from the tree decomposition and make all neighbor bags of $X_{A}$ (except $X_{B}$ ) neighbor of $X_{B}$.

With this in mind, we present Algorithm 4.2 to make a tree decomposition inclusion minimal.

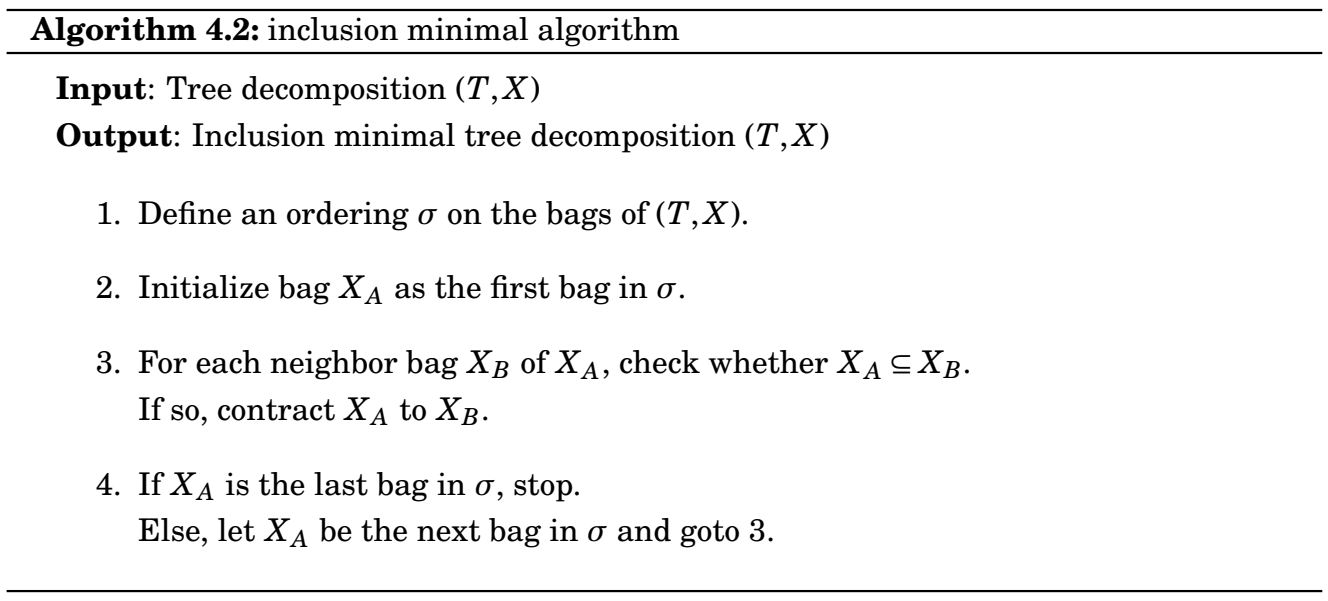

Although we assume inclusion minimality at the start, during a run of MTDA we may still encounter situations where bags are included in neighboring bags. Consider a situation where bag $X_{A}$ is included in $X_{B}$ and there is a fill-in pair in $F_{2}$ that is present in bag $X_{A}$ and in bag $X_{B}$. Then by contracting $X_{A}$ to $X_{B}$, this fill-in pair becomes redundant, i.e. contraction of bags does not maintain fill-in pair minimality. The following lemma shows that new redundant fill-in pairs after a contraction step will only occur in one bag.

Lemma 4.2.5. Let $X_{A} \subseteq X_{B}$ be a bag in tree decomposition $(T, X)$. Then after contracting $X_{A}$ to $X_{B}$, any new elements of $F_{1}$ are present in bag $X_{B}$.

Proof. No new fill-in pairs are generated while contracting bag $X_{A}$ to bag $X_{B}$, so $F_{1} \subseteq F$. Furthermore, the only fill-in pairs for which the number of bags containing them decreases through the contraction were present in $X_{A}$ and thus also in $X_{B}$. Hence, all fill-in pairs that become redundant during the contraction were present only in $X_{A}$ and $X_{B}$ before the contraction. After the contraction, these new redundant fill-in pairs are present only in $X_{B}$.

The following lemma bounds the number of bags in an inclusion minimal tree decomposition from above. 
Lemma 4.2.6. Let $G$ be a graph and let $(T, X)$ be an inclusion minimal tree decomposition of $G$ of width $k$. Then the number of bags in $(T, X)$ is bounded from above by $n-k$.

Proof. Since $w(T, X)=k$, there is a bag $X_{A}$ in $(T, X)$ containing $k+1$ vertices. By inclusion minimality of $(T, X)$, the neighbor bags of $X_{A}$ contain at least one vertex that is not present in $X_{A}$. Clearly, these are different vertices for all neighbors of $X_{A}$ because the path connecting two such neighbors in $T$ crosses $X_{A}$ and $X_{A}$ does not contain the vertices. Likewise, bags at distance 2 from $X_{A}$ contain at least one vertex that is not present in any bag at distance 1 from $X_{A}$ and thus neither in $X_{A}$ itself. Again, these vertices are different from each other. Building on this idea, we conclude that it is possible to choose one vertex from each bag (except bag $X_{A}$ ) that is not present in $X_{A}$, in such a way that all the chosen vertices are pairwise different. Since $X_{A}$ contains $k+1$ vertices, there are only $n-k-1$ different vertices that are not part of $X_{A}$, so there could be at most $n-k-1$ bags besides bag $X_{A}$ in $(T, X)$.

Finally, we make the following easy observation:

Observation 4.2.7. If $X_{A} \subseteq X_{B}$, then the width of $(T, X)$ is invariant under contraction of bag $X_{A}$ to $X_{B}$.

\section{fill-in-pair minimality}

To obtain fill-in-pair minimality in a tree decomposition $(T, X)$, we have to remove the redundant fill-in pairs. It is easy to check the correctness of the following observation about the width of a tree decomposition under removing a redundant fill-in pair.

Observation 4.2.8. The width of tree decomposition $(T, X)$ will not increase under removal of a redundant fill-in pair.

Indeed, the only thing that happens is that one bag is replaced by two bags of strictly smaller size. By Lemma 4.2.3, removal of a redundant fill-in pair will not create new redundant fill-in pairs and even can cause other fill-in pairs to lose their redundancy. Inclusion minimality is not necessarily maintained under removal of a redundant fill-in pair. The following lemma however states that at most two bags violate this property after removing a redundant fill-in pair.

Lemma 4.2.9. After removing a redundant fill-in pair $\{u, v\}$ from an inclusion minimal tree decomposition $(T, X)$ by splitting $X_{M}=T_{\{u, v\}}$ into $X_{M_{u}}$ and $X_{M_{v}}$, bags $X_{M_{u}}$ and $X_{M_{v}}$ are the only bags that may violate the inclusion minimal property.

Proof. Assume that after splitting, bag $X_{A}\left(\neq X_{M_{u}}, X_{M_{v}}\right)$ is included in some bag $X_{B}$. We show that bag $X_{A}$ was already included then in another bag before the split. If $X_{B}=X_{M_{u}}$ or $X_{B}=X_{M_{v}}$, then $X_{A}$ was already included in $X_{M}$. Otherwise $X_{A}$ was already included 
in $X_{B}$. To show that $X_{M_{u}}$ (and $X_{M_{v}}$ ) can indeed be included in another bag after the split, let the tree decomposition before the split be such that $X_{M}$ has a neighbor $X_{C}$ such that $X_{M} \backslash X_{C}=\{v\}$. Then $X_{C}$ contains $u$, so $X_{C}$ will be a neighbor of $X_{M_{u}}$ after the split. Since $v$ was the only element of $X_{M}$ that was not in $X_{C}, X_{M_{u}}$ will be included in $X_{C}$. A similar argument can be used for $X_{M_{v}}$.

\section{minimality}

We showed that the inclusion minimal property might get lost when we try to obtain fill-in pair minimality and vice versa. In this section, we show how to make a tree decomposition minimal. Algorithm MTDA that will be described next assumes as input an inclusion minimal tree decomposition and removes redundant fill-in pairs. After each such removal step, the set of redundant fill-in pairs is updated using Lemma 4.2.3 and inclusion minimality is repaired using Lemma 4.2.9. After repairing inclusion minimality, the set $F_{1}$ of redundant fill-in pairs is again updated using Lemma 4.2.5. By Observations 4.2.7 and 4.2.8, we conclude that application of MTDA to a tree decomposition will not increase its width.

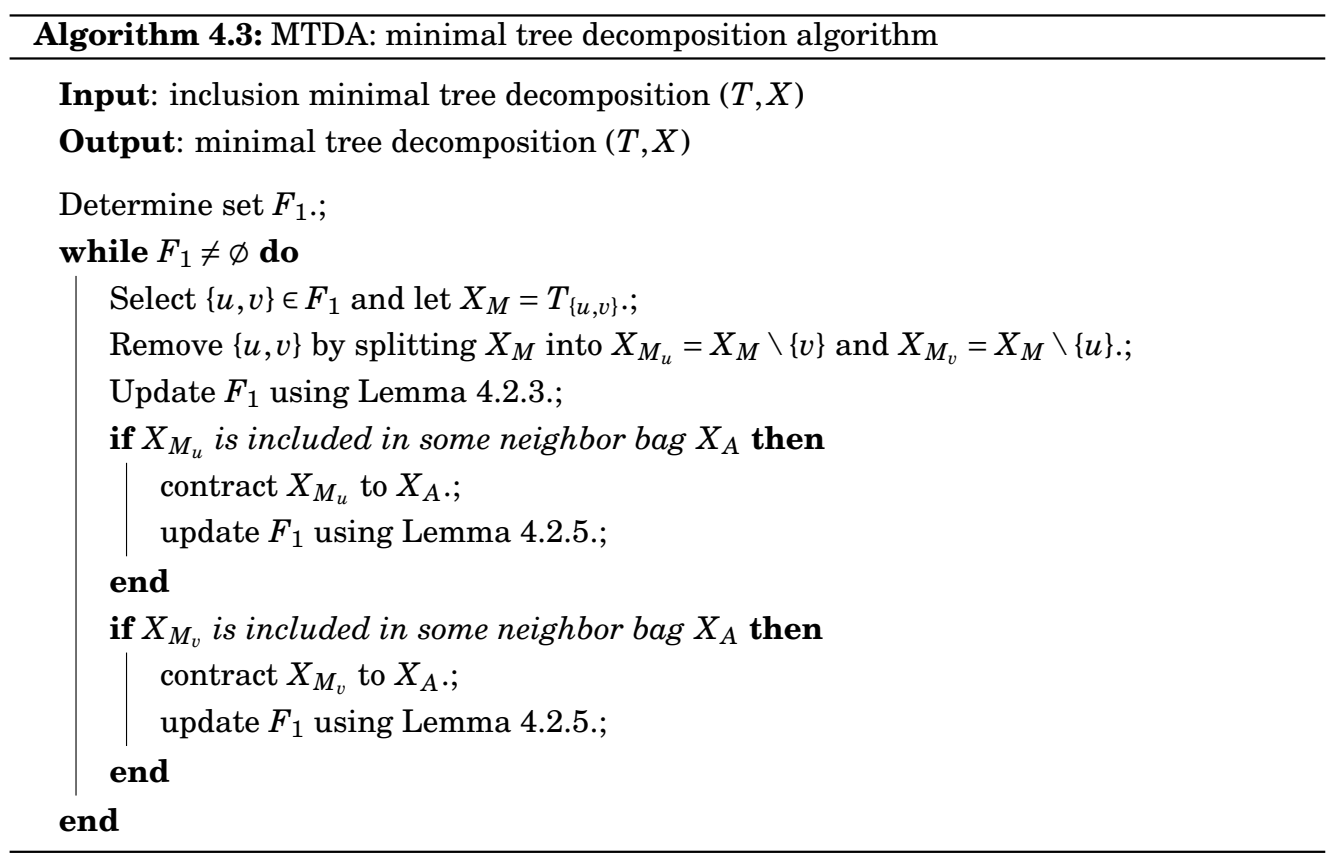

\section{proof of correctness}

Since no new fill-in pairs are created during either removal of a redundant fill-in pair or 
contraction of a bag to a neighboring bag, the set $F$ of fill-in pairs is strictly decreasing in size during a run of Algorithm 4.3. In each run of the while-loop, exactly one fill-in pair leaves the set $F$, namely the redundant fill-in pair that is removed, so after at most $|F|$ iterations of the while-loop, the set $F_{1} \subseteq F$ will be empty and MTDA will stop. At the start and the end of the while-loop, the tree decomposition is inclusion minimal, so MTDA indeed returns a minimal tree decomposition. As a first step, the set of redundant fill-in pairs is determined. If $F_{1}$ is empty, the tree decomposition is minimal and MTDA stops. If $F_{1}$ is non-empty, the algorithm proceeds and selects one of the redundant fill-in pairs in $F_{1}$ that will be removed from the tree decomposition. One for example can choose an element from $F_{1}$ for which the bag containing it is maximal, since this is the bag that will be split into two smaller bags. Note that after removing the selected redundant fill-in pair, the tree decomposition is not necessarily inclusion minimal anymore. The set $F_{1}$ is updated using Lemma 4.2.3. According to Lemma 4.2.9, the only two bags that may violate inclusion minimality are $X_{M_{u}}$ and $X_{M_{v}}$. If $X_{M_{u}}$ is contained in one of its neighbors, it is therefore contracted to this neighbor. By performing this contraction, the set $F_{1}$ might change and we use Lemma 4.2.5 to update $F_{1}$. If $X_{M_{v}}$ is included in one of its neighbors, it is also contracted to that neighbor and again Lemma 4.2.5 is used to update $F_{1}$. After this, the tree decomposition is inclusion minimal again and $F_{1}$ again contains all the redundant fill-in pairs. If $F_{1}$ is non-empty, MTDA continues and selects another element from $F_{1}$ to be removed.

Theorem 4.2.10. Algorithm 4.3 runs in $O(f(e+f))$ time.

Proof. As we already observed, the number of fill-in pairs will strictly decrease during a run of MTDA. At the start of MTDA, there are $f=|F|$ such fill-in pairs and only these $f$ fill-in pairs are potential elements of $F_{1}$ during a run of MTDA. Therefore, the while-loop will be executed at most $f$ times during a run of MTDA. It remains to show that one iteration of the while-loop takes $\mathrm{O}(e+f)$ time. When removing a redundant fill-in pair $\{u, v\}$ by splitting bag $X_{M}$ into $X_{M_{u}}$ and $X_{M_{v}}$, each neighbor bag of $X_{M}$ is attached to $X_{M_{u}}$, and then those containing $v$ are reattached to $X_{M_{v}}$. Checking for the presence of vertex $v$ in all neighbor bags of $X_{M}$ can be done in $\mathrm{O}(n)$ time. To update the set $F_{1}$ using Lemma 4.2.3, each element of $F_{1}$ that was present only in bag $X_{M}$ has to be checked for incidence to both $u$ and $v$, a process that takes complexity $\mathrm{O}(f)$. Then the new bags $X_{M_{u}}$ and $X_{M_{v}}$ are checked for inclusion in one of their neighbors, which takes $\mathrm{O}(n)$ time. Finally, the set $F_{1}$ is updated again by checking at most two bags for new redundant fill-in pairs. This can be done by checking for all vertex pairs in these bags that are not in $E$, whether they are present only in the bag under consideration, taking $\mathrm{O}(e+f)$ time. Assuming that $n<e+f$ (which is true for any connected graph but a tree), we conclude that one iteration of the while-loop takes $\mathrm{O}(e+f)$ time, making the time complexity of MTDA equal to $\mathrm{O}(f(e+f))$. 


\subsection{Local search}

We showed how a regular fill-in pair can be removed from a minimal tree decomposition by Algorithm 4.1 and how this could introduce new redundant fill-in pairs. We also showed how Algorithm 4.3 can turn such a non-minimal tree decomposition back into a minimal one with lower or equal width. In this section, the two procedures will be combined into a local search heuristic for upper bounding the treewidth of the input graph.

\subsection{1 starting solution}

As a starting solution for the local search heuristic, any upper bound heuristic for treewidth can be used. We apply our local search heuristic on two different starting solutions for each considered graph instance. The first starting solution is obtained via a heuristic called Greedy Fill-In (GFI). GFI was first described in (97). It can be applied directly to graph $G$ and returns a chordalization $H=(V, E \cup F)$ of $G$ by repeatedly selecting the vertex for which the least fill-in edges have to be added when it is eliminated, then turning these neighbors into a clique by adding fill-in edges, and finally removing that vertex from consideration. The obtain a second starting solution, we employed an algorithm that is know as the lexicographic breadth-first search recognition algorithm, introduced in (98). Originally, this algorithm was constructed to recognize chordality of an input graph $G$ and it terminated at the moment it recognized that no perfect elimination ordering can be found. Without the termination step however, it returns an elimination ordering on the vertices of $G$ that can be used to construct a chordalization $H=(V, E \cup F)$ of $G$. We use a variant of this algorithm, known as LEX_M, that guarantees that the returned chordalization is minimal. For computational evaluations of GFI and LEX_M, we refer to respectively (36) and (83).

The chordalization that is returned by either GFI or LEX_M can be turned into an inclusion minimal tree decomposition $(T, X)$ of the same width by means of Algorithm 2.3. Note that the set $F$ of fill-in pairs in $(T, X)$ equals the set $F$ of fill-in edges in chordalization $H=(V, E \cup F)$. Tree decompositions that are constructed via LEX_M are therefore minimal. The ones that are obtained via GFI are not necessarily fill-in pair minimal, so we apply Algorithm 4.3 to them in order to obtain minimal starting solutions for our local search heuristic.

\subsection{2 neighborhood}

Definition 4.3.1. Given a graph $G$ and a minimal tree decomposition $(T, X)_{0}$, the neighborhood of $(T, X)_{0}$ is the set of minimal tree decompositions of $G$ that can be obtained from $(T, X)_{0}$ by a single application of Algorithm 4.1 followed by a single application of Algorithm 4.3 . 
Note that Algorithm 4.1 applied to a minimal tree decomposition does not necessarily return an inclusion minimal tree decomposition. To obtain the inclusion minimal tree decomposition that is requested as input for Algorithm 4.3, we apply Algorithm 4.2 once.

The neighborhood as defined here is quite large in the sense that each element of $F$ is a candidate for removal in Algorithm 4.1. We present some ideas here for how to decrease the size of the neighborhood.

First of all, to reduce the size of the largest bag, MTDA should remove redundant fillin pairs from the largest bag. In order to do so, there should be redundant fill-in pairs in the largest bag at the moment MTDA is applied. To achieve this, we apply Algorithm 4.1 to a fill-in pair that is present in a maximal bag of the minimal tree decomposition. This is done by merging its subtree into a single bag $X_{M}$ and then splitting $X_{M}$ into two bags, which are then maximal bags of the tree decomposition and moreover the only bags that possibly contain redundant fill-in pairs. Even when at the start of Algorithm 4.3 there are no redundant fill-in pairs in a maximal bag, there might still be redundant fill-in pairs in a maximal bag in a later stage of the algorithm. However these are more difficult to predict, so we apply Algorithm 4.1 only to fill-in pairs that are present in a maximal bag of the minimal tree decomposition.

Secondly, we realize that most of the minimal tree decompositions in the neighborhood of a minimal tree decomposition $(T, X)$ will have a higher width than $(T, X)$. Especially when $T_{\{u, v\}}$ contains a lot of vertices, application of Algorithm 4.1 to fill-in pair $\{u, v\}$ can dramatically increase the width of the tree decomposition. Most likely, the subsequent decrease in width caused by Algorithm 4.3 will not make up for this increase. Therefore it seems appropriate to apply Algorithm 4.1 only on fill-in pairs $\{u, v\}$ for which the number of different vertices in $T_{\{u, v\}}$ is reasonably small.

Combining the foregoing two considerations, we restrict the neighborhood of a minimal tree decomposition $(T, X)$ to solutions that can be obtained from $(T, X)$ by the following two operations in this order:

1. Single application of Algorithm 4.1 to a fill-in pair $\{u, v\}$ that is present in a maximal bag of $(T, X)$ and for which $T_{\{u, v\}}$ contains at most $k * w$ vertices, where $w$ is the width of $(T, X)$ and $1<k \leq 2$ is a constant.

2. Single application of Algorithm 4.3 to restore minimality.

Finally, we mention that although different fill-in pairs may be removed in the first step, the application of MTDA could cause the resulting minimal tree decompositions to be equal. Being able to detect in advance whether two fill-in pairs being removed in the first step will lead to the same minimal tree decomposition would clearly be very helpful, since it would allow us to avoid exploring a single solution in the neighborhood more than once. In this chapter however, we did not further explore this consideration. 


\subsection{3 solution improvements}

Clearly, we are interested in minimal tree decompositions in the (restricted) neighborhood of $(T, X)$ that have a width strictly lower than $(T, X)$. These are the tree decompositions for which the increase in width (caused by Algorithm 4.1) is strictly smaller than the subsequent decrease in width (caused by Algorithm 4.3). However, sometimes the local search heuristic is able to reduce the size of a maximal bag in one part of the tree decomposition, while in another part a maximal bag remains untouched. This would remain unnoticed if we only consider the width of the resulting tree decomposition. For this reason we consider solutions in the neighborhood of $(T, X)$ that have the same width as $(T, X)$ but a strictly smaller number of maximal bags, also as improvements over $(T, X)$. A third measure for improvement can be stated in terms of the number of fill-in pairs. Suppose that a solution in the neighborhood of $(T, X)$ has the same width and the same number of maximal bags as $(T, X)$, but a larger number of fill-in pairs. Then we consider this solution to be an improvement over $(T, X)$ as well, since a larger number of fill-in pairs basically means a larger neighborhood and thus more potential for better solutions in the neighborhood. In our heuristic, we apply a first improving strategy, i.e. as soon as a better solution is found, the current best solution will be updated and the search for further improvements is continued in the neighborhood of the new best solution.

\subsection{Experimental results}

In this section some experimental results of the local search heuristic are presented. Implementation was done in $C++$ and all experiments were run on an AMD Athlon 2400XP+ with $1 \mathrm{~Gb}$ of RAM. Graphs from Frequency Assignment networks and Second Dimacs graph coloring challenge are used as input graphs. Upper bound heuristics GFI and LEX-M are used to obtain starting solutions and we restrict the neighborhood to solutions that can be obtained by applying Algorithm 4.1 in the first step to fill-in pairs $\{u, v\}$ that are present in a maximal bag and for which the number of vertices in $T_{\{u, v\}}$ exceeds the size of this maximal bag by at most 10. To improve the chances of finding a better solution quickly, neighbors for which the number of vertices in $T_{\{u, v\}}$ are smallest are explored first. Our tables use the following terminology. $N$ and $E$ respectively denote the number of vertices and edges in the input graph. The columns $l b$ and $u b$ give the best upper and lower bounds on treewidth as reported by Koster et al. (see (83)) in their rigorous computational study on upper bound heuristics like greedy fill-in, min-fill, max-cardinality search and the minimum-separatingvertex-set heuristic and several lower bound heuristics. The columns $g$ f $i$ and $l e x-m$ denote the width of the starting solution as obtained by upper bound heuristic GFI and LEX-M. Finally, the columns $L S$ give the upper bound on treewidth that our local search heuristic 
returned using these starting solutions in time seconds.

\subsubsection{Dimacs graph coloring}

The statistics generated by our local search on the instances from the Dimacs Graph Coloring challenge can be found in Table 4.1. We were able to improve on the best known upper bound for the following graphs: games120, all of the le450-instances, queen6_6 to queen14_14, school1, zeroin.i.2 and zeroin.i.3, while for most other graphs we were able to match the best known upper bounds. For some instances (le450-5c, le450-25a, queen10_10), the portion of the gap between lower and upper bound that was closed is considerable. For the le450-graphs, upper bound heuristic LEX-M did not return an upper bound within an hour, so we decided to apply our local search heuristic for these graphs only with the starting solution provided by GFI.

\subsection{2 frequency assignment}

As a second set of graphs, we took the instance set of the CALMA project on frequency assignment problems. The results of our tests are displayed in Table 4.2. For graph celar03 we were able to close the gap between upper and lower bound. For all instances from graph02 to graph14, we were able to improve on the upper bound on its treewidth, often drastically. LEX-M could not find an upper bound on the treewidth of graph11 to graph14 within an hour, for which reason we only ran our local search heuristic on these graphs with a starting solution obtained by GFI.

\subsubsection{Bayesian networks}

Instances from the Bayesian Network Repository formed our final testing set. The results of the experiment can be found in Table 4.3. 


\begin{tabular}{|c|c|c|c|c|c|c|c|c|c|c|}
\hline Graph & $\mathbf{N}$ & $\mathbf{E}$ & lb & $\mathbf{u b}$ & gfi & LS & time & lex-m & LS & time \\
\hline anna & 138 & 493 & 12 & 12 & 12 & 12 & 0.17 & 12 & 12 & 0.31 \\
\hline david & 87 & 406 & 12 & 13 & 13 & 13 & 0.10 & 13 & 13 & 0.10 \\
\hline fpsol2.i.1 & 269 & 11654 & 66 & 66 & 66 & 66 & 0.58 & 66 & 66 & 0.70 \\
\hline fpsol2.i.2 & 363 & 8691 & 31 & 31 & 31 & 31 & 2.05 & 52 & 31 & 0.71 \\
\hline fpsol2.i.3 & 363 & 8688 & 31 & 31 & 31 & 31 & 2.11 & 52 & 31 & 0.69 \\
\hline games120 & 120 & 638 & 24 & 36 & 40 & 35 & 141.76 & 37 & 32 & 561.25 \\
\hline homer & 556 & 1628 & 26 & 31 & 31 & 31 & 99.55 & 36 & 31 & 45.22 \\
\hline huck & 74 & 301 & 10 & 10 & 10 & 10 & 0.00 & 10 & 10 & 0.00 \\
\hline inithx.i.1 & 519 & 18707 & 56 & 56 & 56 & 56 & 27.41 & 223 & 56 & 1021.58 \\
\hline inithx.i.2 & 558 & 13979 & 31 & 31 & 31 & 31 & 94.17 & 227 & 31 & 1075.75 \\
\hline inithx.i.3 & 559 & 13969 & 31 & 31 & 31 & 31 & 93.58 & 227 & 31 & 1066.48 \\
\hline jean & 77 & 254 & 9 & 9 & 9 & 9 & 0.00 & 9 & 9 & 0.01 \\
\hline le450-5a & 450 & 5714 & 79 & 308 & 315 & 303 & 11929.50 & - & - & - \\
\hline le $450-5 b$ & 450 & 5734 & 79 & 307 & 318 & 303 & 10519.11 & - & - & - \\
\hline le450-5c & 450 & 9803 & 106 & 315 & 315 & 296 & 10076.76 & - & - & - \\
\hline le $450-5 d$ & 450 & 9757 & 106 & 295 & 299 & 285 & 12352.52 & - & - & - \\
\hline le450-15a & 450 & 8168 & 94 & 290 & 290 & 276 & 10314.78 & - & - & - \\
\hline le450-15b & 450 & 8169 & 95 & 291 & 301 & 287 & 12337.49 & - & - & - \\
\hline le450-15c & 450 & 16680 & 139 & 373 & 377 & 366 & 17365.87 & - & - & - \\
\hline le450-15d & 450 & 16750 & 141 & 375 & 375 & 369 & 10474.78 & - & - & - \\
\hline le $450-25 a$ & 450 & 8260 & 96 & 252 & 258 & 232 & 10348.08 & - & - & - \\
\hline le $450-25 b$ & 450 & 8263 & 96 & 255 & 265 & 247 & 17352.31 & - & - & - \\
\hline le450-25c & 450 & 17343 & 144 & 353 & 353 & 341 & 13817.04 & - & - & - \\
\hline le450-25d & 450 & 17425 & 143 & 352 & 363 & 351 & 13063.89 & - & - & - \\
\hline miles 250 & 125 & 387 & 9 & 9 & 9 & 9 & 0.06 & 10 & 9 & 0.09 \\
\hline miles500 & 128 & 1170 & 22 & 22 & 23 & 22 & 9.07 & 22 & 22 & 1.43 \\
\hline miles750 & 128 & 2113 & 35 & 36 & 39 & 36 & 9.44 & 36 & 36 & 15.63 \\
\hline miles 1000 & 128 & 3216 & 49 & 49 & 50 & 49 & 7.78 & 49 & 49 & 6.84 \\
\hline miles 1500 & 128 & 5198 & 77 & 77 & 77 & 77 & 3.58 & 77 & 77 & 2.83 \\
\hline mulsol.i.1 & 138 & 3925 & 50 & 50 & 50 & 50 & 0.33 & 66 & 50 & 1.43 \\
\hline mulsol.i.2 & 173 & 3885 & 32 & 32 & 32 & 32 & 0.17 & 69 & 32 & 1.82 \\
\hline mulsol.i.3 & 174 & 3916 & 32 & 32 & 32 & 32 & 0.19 & 69 & 32 & 1.87 \\
\hline mulsol.i.4 & 175 & 3946 & 32 & 32 & 32 & 32 & 0.19 & 69 & 32 & 1.82 \\
\hline mulsol.i.5 & 176 & 3973 & 31 & 31 & 32 & 31 & 0.88 & 69 & 31 & 1.97 \\
\hline myciel3 & 11 & 20 & 5 & 5 & 5 & 5 & 0.01 & 5 & 5 & 0.02 \\
\hline myciel4 & 23 & 71 & 9 & 10 & 11 & 10 & 0.26 & 12 & 10 & 0.32 \\
\hline myciel5 & 47 & 236 & 16 & 19 & 21 & 19 & 3.95 & 25 & 19 & 11.55 \\
\hline myciel6 & 95 & 755 & 29 & 35 & 35 & 35 & 36.52 & 55 & 35 & 246.39 \\
\hline myciel7 & 191 & 2360 & 52 & 66 & 66 & 66 & 232.89 & 103 & 78 & 2126.17 \\
\hline queen5_5 & 25 & 160 & 14 & 18 & 18 & 18 & 0.16 & 18 & 18 & 0.29 \\
\hline queen6_6 & 36 & 290 & 18 & 26 & 26 & 25 & 4.33 & 26 & 25 & 4.18 \\
\hline queen7_7 & 49 & 476 & 22 & 36 & 37 & 35 & 40.94 & 36 & 35 & 15.79 \\
\hline queen8_8 & 64 & 728 & 28 & 47 & 48 & 46 & 78.19 & 47 & 45 & 88.05 \\
\hline queen9_9 & 81 & 1056 & 35 & 59 & 65 & 58 & 403.95 & 61 & 60 & 269.99 \\
\hline queen10_10 & 100 & 1470 & 42 & 75 & 79 & 72 & 653.95 & 75 & 74 & 499.49 \\
\hline queen11_11 & 121 & 1980 & 48 & 90 & 95 & 89 & 1615.43 & 92 & 91 & 567.80 \\
\hline queen12_12 & 144 & 2596 & 55 & 109 & 117 & 108 & 2338.92 & 110 & 104 & 4019.00 \\
\hline queen13_13 & 169 & 3328 & 61 & 126 & 137 & 124 & 12013.44 & 126 & 126 & 1025.41 \\
\hline queen14_14 & 196 & 4186 & 67 & 149 & 163 & 146 & 5802.14 & 151 & 148 & 7340.12 \\
\hline queen15_15 & 225 & 5180 & 73 & 171 & 183 & 174 & 3399.04 & 172 & 166 & 5590.31 \\
\hline queen16_16 & 256 & 6320 & 79 & 194 & 218 & 196 & 11082.09 & 196 & 189 & 12334.27 \\
\hline school1 & 385 & 19095 & 149 & 221 & 225 & 213 & 6049.54 & 226 & 192 & 12131.81 \\
\hline zeroin.i.1 & 126 & 4100 & 50 & 50 & 50 & 50 & 0.20 & 50 & 50 & 0.21 \\
\hline zeroin.i.2 & 157 & 3541 & 32 & 33 & 33 & 32 & 2.65 & 43 & 32 & 5.21 \\
\hline zeroin.i.3 & 157 & 3540 & 32 & 33 & 33 & 32 & 1.89 & 43 & 32 & 5.14 \\
\hline
\end{tabular}

Table 4.1: Dimacs Graph Coloring instances 


\begin{tabular}{|l||c|c||c|c||r|r|r||r|r|r|}
\hline Graph & N & $\mathbf{E}$ & lb & ub & gfi & LS & time & lex-m & LS & time \\
\hline \hline celar01 & 458 & 1449 & 15 & 15 & 16 & 15 & 12.72 & 18 & 15 & 13.08 \\
\hline celar02 & 100 & 311 & 10 & 10 & 10 & 10 & 0.04 & 10 & 10 & 0.04 \\
\hline celar03 & 200 & 721 & 14 & 15 & 15 & 14 & 12.18 & 16 & 14 & 3.45 \\
\hline celar04 & 340 & 1009 & 15 & 16 & 17 & 16 & 2.50 & 16 & 16 & 7.97 \\
\hline celar05 & 200 & 681 & 14 & 15 & 16 & 15 & 2.91 & 16 & 15 & 3.52 \\
\hline celar06 & 100 & 350 & 11 & 11 & 11 & 11 & 0.03 & 11 & 11 & 0.02 \\
\hline celar07 & 200 & 817 & 16 & 16 & 16 & 16 & 2.57 & 18 & 16 & 5.72 \\
\hline celar08 & 458 & 1655 & 16 & 16 & 16 & 16 & 9.01 & 20 & 17 & 3.40 \\
\hline celar09 & 340 & 1130 & 16 & 16 & 16 & 16 & 3.08 & 18 & 16 & 12.77 \\
\hline celar10 & 340 & 1130 & 16 & 16 & 16 & 16 & 3.11 & 18 & 16 & 12.66 \\
\hline celar11 & 340 & 975 & 14 & 15 & 15 & 15 & 1.10 & 16 & 15 & 2.32 \\
\hline graph01 & 100 & 358 & 16 & 24 & 25 & 24 & 28.26 & 27 & 23 & 417.46 \\
\hline graph02 & 200 & 709 & 24 & 50 & 51 & 41 & 305.03 & 57 & 43 & 1132.21 \\
\hline graph03 & 100 & 340 & 16 & 21 & 21 & 20 & 79.86 & 27 & 20 & 266.48 \\
\hline graph04 & 200 & 734 & 24 & 55 & 57 & 48 & 940.49 & 60 & 51 & 932.48 \\
\hline graph05 & 100 & 416 & 18 & 25 & 26 & 24 & 31.99 & 27 & 24 & 141.16 \\
\hline graph06 & 200 & 843 & 26 & 53 & 58 & 51 & 826.40 & 60 & 55 & 2429.09 \\
\hline graph07 & 200 & 843 & 26 & 53 & 58 & 51 & 826.63 & 60 & 55 & 2433.60 \\
\hline graph08 & 340 & 1234 & 32 & 91 & 95 & 84 & 1706.64 & 105 & 92 & 12077.96 \\
\hline graph09 & 458 & 1667 & 37 & 116 & 119 & 107 & 8415.20 & 132 & 121 & 12234.54 \\
\hline graph10 & 340 & 1275 & 31 & 93 & 97 & 84 & 12007.97 & 105 & 93 & 12123.45 \\
\hline graph11 & 340 & 1425 & 34 & 97 & 97 & 83 & 12079.65 & - & - & - \\
\hline graph12 & 340 & 1256 & 31 & 86 & 86 & 77 & 5235.54 & - & - & - \\
\hline graph13 & 458 & 1877 & 39 & 126 & 135 & 120 & 12130.80 & - & - & - \\
\hline graph14 & 458 & 1398 & 34 & 121 & 123 & 112 & 12041.12 & - & - & - \\
\hline & & & & & & & & & & \\
\hline
\end{tabular}

Table 4.2: CALMA Project on Frequency Assignment instances 
Chapter 4. Local Search

\begin{tabular}{|l||c|c||c|c||c|c|r||r|r|r|}
\hline Graph & N & $\mathbf{E}$ & lb & ub & gfi & LS & time & lex-m & LS & time \\
\hline \hline alarm & 37 & 65 & 4 & 4 & 4 & 4 & 0.00 & 4 & 4 & 0.00 \\
\hline barley & 48 & 126 & 6 & 7 & 7 & 7 & 0.03 & 7 & 7 & 0.06 \\
\hline boblo & 221 & 328 & 3 & 3 & 3 & 3 & 0.13 & 4 & 3 & 0.12 \\
\hline diabetes & 413 & 819 & 4 & 4 & 4 & 4 & 13.27 & 35 & 4 & 128.07 \\
\hline fungiuk & 15 & 36 & 4 & 4 & 4 & 4 & 0.00 & 4 & 4 & 0.00 \\
\hline link & 724 & 1738 & 12 & 13 & 15 & 13 & 16.37 & 37 & 29 & 592.47 \\
\hline mainuk & 48 & 198 & 7 & 7 & 7 & 7 & 0.02 & 7 & 7 & 0.07 \\
\hline mildew & 35 & 80 & 4 & 4 & 4 & 4 & 0.03 & 4 & 4 & 0.03 \\
\hline munin1 & 189 & 366 & 10 & 11 & 11 & 11 & 0.91 & 15 & 11 & 11.08 \\
\hline munin2 & 1003 & 1662 & 6 & 7 & 7 & 7 & 22.73 & 16 & 7 & 178.24 \\
\hline munin3 & 1044 & 1745 & 7 & 7 & 7 & 7 & 6.23 & 15 & 8 & 95.07 \\
\hline munin4 & 1041 & 1843 & 8 & 8 & 8 & 8 & 10.01 & 28 & 8 & 76.93 \\
\hline munin-kgo & 1066 & 1730 & 5 & 5 & 5 & 5 & 2.77 & 13 & 6 & 12.37 \\
\hline oesoca & 39 & 67 & 3 & 3 & 3 & 3 & 0.00 & 3 & 3 & 0.00 \\
\hline oesoca42 & 42 & 72 & 3 & 3 & 3 & 3 & 0.00 & 3 & 3 & 0.00 \\
\hline oow-bas & 27 & 54 & 4 & 4 & 4 & 4 & 0.02 & 4 & 4 & 0.02 \\
\hline oow-solo & 40 & 87 & 5 & 6 & 6 & 6 & 0.16 & 6 & 6 & 0.42 \\
\hline oow-trad & 33 & 72 & 5 & 6 & 6 & 6 & 0.04 & 6 & 6 & 0.08 \\
\hline pathfinder & 109 & 211 & 6 & 6 & 6 & 6 & 0.07 & 7 & 6 & 0.05 \\
\hline pignet2 & 3032 & 7264 & 48 & 135 & 143 & 127 & 12391.09 & - & - & - \\
\hline pigs & 441 & 806 & 8 & 10 & 10 & 9 & 7.02 & 18 & 10 & 20.94 \\
\hline ship-ship & 50 & 114 & 6 & 8 & 8 & 8 & 0.14 & 9 & 8 & 0.74 \\
\hline vsd & 38 & 62 & 4 & 4 & 4 & 4 & 0.00 & 4 & 4 & 0.01 \\
\hline water & 32 & 123 & 9 & 9 & 10 & 9 & 0.19 & 10 & 10 & 0.06 \\
\hline weeduk & 15 & 49 & 7 & 7 & 7 & 7 & 0.00 & 7 & 7 & 0.00 \\
\hline wilson & 21 & 27 & 3 & 3 & 3 & 3 & 0.00 & 3 & 3 & 0.01 \\
\hline
\end{tabular}

Table 4.3: Bayesian Network Repository instances 


\section{Chapter 5}

\section{Grid Minors}

This chapter of the thesis is dedicated to treewidth in the class of planar graphs. More specifically, we examine the relation between treewidth and two other graph parameters, namely branchwidth and the side size of a largest square-grid minor in some special classes of planar graphs.

The complexity of finding the treewidth in planar graphs is not known, but it is widely believed that the problem is $N P$-hard. Approximating the treewidth of planar graphs is therefore an interesting research direction. To the best of our knowledge, the most successful approximations to the treewidth in planar graphs are based on two well known lower bounds, the branchwidth and the side size of the largest square-grid minor.

Branchwidth is a notion that is very closely related to treewidth and, just as for treewidth, it is an important algorithmic concept that is widely used in discrete mathematics and theoretical computer science; see, e.g., Bodlaender (30) and Hicks (74). Gu and Tamaki (69) constructed a $\mathrm{O}\left(n^{3}\right)$ algorithm to compute optimal branch decompositions for planar graphs. For more work on planar branch decompositions, we refer to $(14 ; 72 ; 73)$. As for the relation between treewidth $(t w)$ and branchwidth $(b w)$, Robertson and Seymour show in (96) that for any graph $G$ with $b w(G)>1$ it holds that $b w(G) \leq t w(G)+1 \leq\left\lfloor\frac{3}{2} b w(G)\right\rfloor$. Seymour and Thomas developed an $\mathrm{O}\left(n^{3}\right)$ time algorithm in (101) finding the minimum branchwidth of a given planar graph on $n$ vertices. Therefore we can approximate treewidth efficiently in planar graphs to a factor $\frac{3}{2}$ from optimal.

Concerning the relation between treewidth and grid minors in planar graphs, independently in (67) and (106) it was shown that the treewidth of a planar graph is at most 5 times the side size of a largest square-grid minor in the graph. This upper bound on the treewidth was improved in (70) to 4.5 times the side size of a largest square-grid minor. The problem of finding the largest square-grid minor in a planar graph is interesting in itself. Although it is not known whether the problem can be solved in polynomial time, there is a $\mathrm{O}\left(n^{2} \log n\right)$ time algorithm for finding a square-grid minor with side size at least one fourth of the side 
size of the largest square-grid minor, see (35).

The definition of branchwidth (see Section 5.1) straightforwardly implies that the side size of the largest square-grid minor $(\mathrm{gm})$ is bounded from above by the branchwidth in any graph. In (56), a short proof can be found for the fact that graphs with high branchwidth contain large grid minors. In this chapter we further analyze relationships between treewidth, branchwidth and the side size of the largest square-grid minor in planar graphs. We present a class of planar graphs for which $t w \approx \frac{3 g m}{2}-1$ and $b w=g m$. We experienced that this seemingly trivial task is quite challenging because there are no simple techniques to accurately estimate treewidth in planar graphs. For the presented graph family, the branchwidth is easily verifiable, while arguing why the treewidth in the presented graph class is large is quite technical and requires methods from graph minor theory.

We present two different ways to determine the parameter $g m$ in our graph class. We see the contribution of this research therefore not only in the construction of lower bounds for the treewidth approximation, but also in the proof methodologies for determining the parameter $g m$ in a planar graph.

Furthermore we introduce two classes of planar graphs for which we conjecture that both branchwidth and treewidth are roughly equal to $2 \mathrm{gm}$. We do believe that these classes are worst cases for branchwidth and treewidth approximation in terms of $g \mathrm{~m}$ in planar graphs.

The chapter is organized as follows. In Section 5.1, some new notions and definitions will be put forward. Subsequently in Section 5.2 we introduce a class of planar graphs for which we were able to determine $b w, t w$ and $g m$. Two more classes of planar graphs are introduced in Section 5.3 for which several upper and lower bounds on $b w, t w$ and $g m$ are presented. Finally, we summarize the results from this chapter in Section 5.4 in which we also pose some open questions.

The content of this chapter is based on cooperation with Alexander Grigoriev and Natalya Usotskaya.

\subsection{Preliminaries}

For $n, m \geq 2$, the $(n \times m)$-grid graph (see (96)) is the simple graph with vertices $v_{i j}(1 \leq i \leq$ $n, 1 \leq j \leq m$ ) where $v_{i j}$ and $v_{i^{\prime} j^{\prime}}$ are adjacent if $\left|i-i^{\prime}\right|+\left|j-j^{\prime}\right|=1$. In this chapter, we are interested only in square grids. For simplicity of notation, we refer to the square $(n \times n)$-grid graph simply as the $n$-grid. In an $n$-grid, $n$ is referred to as the side size of the $n$-grid.

We recall that $H$ is a minor of a graph $G$ if $H$ is obtainable from a subgraph of $G$ by edge contractions. A minor of graph $G$ that forms a square grid graph is called a square-grid minor of $G$. The side size of the largest square-grid minor of $G$ will be denoted by $\operatorname{gm}(G)$.

We continue with definitions of branchwidth and branch decompositions. Both notions were introduced in (96). 
Definition 5.1.1. $A$ branch decomposition of a graph $G=(V, E)$ is a pair $(T, \tau)$, where $T$ is a ternary tree (every vertex has degree 1 or 3 ) and $\tau$ is a bijection from the set of leaves of $T$ to $E(G)$.

The order of an edge $e$ of tree $T$ in a branch decomposition is the number of vertices $v$ from $V(G)$ such that there are leaves $t_{1}, t_{2}$ of $T$ in different components of $T \backslash e$ for which $\tau\left(t_{1}\right), \tau\left(t_{2}\right)$ are both incident to $v$. The width of branch decomposition $(T, \tau)$ is the maximum order over all edges of $T$.

Definition 5.1.2. The branchwidth $b w(G)$ of graph $G$ is the minimum width over all branch decompositions of $G$ (or 0 if $|E(G)| \leq 1$, when $G$ has no branch decompositions).

Two subsets $V^{\prime}, V^{\prime \prime} \subseteq V$ in $G=(V, E)$ are said to touch each other if either they have a vertex in common or $E$ contains an edge $u v$ with $u \in V^{\prime}$ and $v \in V^{\prime \prime}$. Given a graph $G=(V, E)$, we say that $V^{\prime} \subseteq V$ is connected if $G\left[V^{\prime}\right]$ is connected, see Section 2.1.

Definition 5.1.3. A set $\mathscr{B}$ of mutually touching, connected subsets of $V$ is called a bramble of $G$. A subset of $V$ intersecting with every element of $\mathscr{B}$ is called a hitting set for bramble $\mathscr{B}$.

The order of a bramble $\mathscr{B}$ is the minimum size over all hitting sets for $\mathscr{B}$.

Definition 5.1.4. The bramble number of a graph $G$ is the maximum order over all brambles $\mathscr{B}$ of $G$.

Brambles are a useful tool in bounding treewidth from below as can be concluded from the following theorem that is due to Seymour and Thomas (100) and its corollary.

Theorem 5.1.5. Let $k$ be a non-negative integer. A graph has treewidth $k$ if and only if it has bramble number $k+1$.

Corollary 5.1.6. Given graph $G$ and a bramble $\mathscr{B}$ of order $k, t w(G) \geq k-1$.

Given a planar graph $G$ together with its planar embedding, two faces $f^{\prime}$ and $f^{\prime \prime}$ in the embedding are said to be adjacent if there is a vertex in $V(G)$ incident to both $f^{\prime}$ and $f^{\prime \prime}$. We call a sequence $f_{1}, \ldots, f_{n}$ of faces an $f_{1} f_{n}$-facepath if each of the faces in the sequence is adjacent to the previous face and to the next face in the sequence. The length of a facepath is equal to the number of faces in the facepath minus one.

Given an embedding of a graph $G$, we denote the collection of faces that are incident to vertex $v$ by $F_{v}$. The face distance between faces $f^{\prime}$ and $f^{\prime \prime}$ in the embedding, denoted by $d_{G}\left(f^{\prime}, f^{\prime \prime}\right)$ in this thesis, is equal to the length of the shortest $f^{\prime} f^{\prime \prime}$-facepath. The distance between vertex $v$ and face $f^{\prime}$, denoted by $d_{G}\left(v, f^{\prime}\right)$, is equal to the length of a shortest $f^{\prime} f^{\prime \prime}$ facepath, over all faces $f^{\prime \prime} \in F_{v}$. It can be shown that for the considered planar graph families 
both distances are independent of the embedding of $G$, since they are almost 3-connected; see (39).

For two graphs $G$ and $H$, the cartesian product $G \times H$ is a graph with $V(G \times H)=V(G) \times$ $V(H), E(G \times H)=\left\{\left\{\left(u, v_{1}\right),\left(u, v_{2}\right)\right\} \mid u \in V(G),\left\{v_{1}, v_{2}\right\} \in E(H)\right\}$ $\bigcup\left\{\left\{\left(u_{1}, v\right),\left(u_{2}, v\right)\right\} \mid\left\{u_{1}, u_{2}\right\} \in E(G), v \in V(H)\right\}$. The following definition of a cylinder graph will be used in Section 5.3 and originates from (94).

Definition 5.1.7. For cycle $C_{k}$ and path $P_{h}$, we call $C_{k} \times P_{h} a k \times h$ cylinder and we denote it by $C_{k, h}$.

\subsection{X-grids}

In this section we start by introducing a family $X_{i}$ of planar graphs, which we will call $\mathrm{X}$-grids. Graph $X_{n}$ from this family can be constructed by taking an $n$-grid, two $(n \times\lceil n / 2\rceil)$ grids and two $(\lceil n / 2\rceil \times n)$-grids. The four rectangular grids are connected via their long sides to the four sides of the square grid, as is illustrated in Figure 5.1.

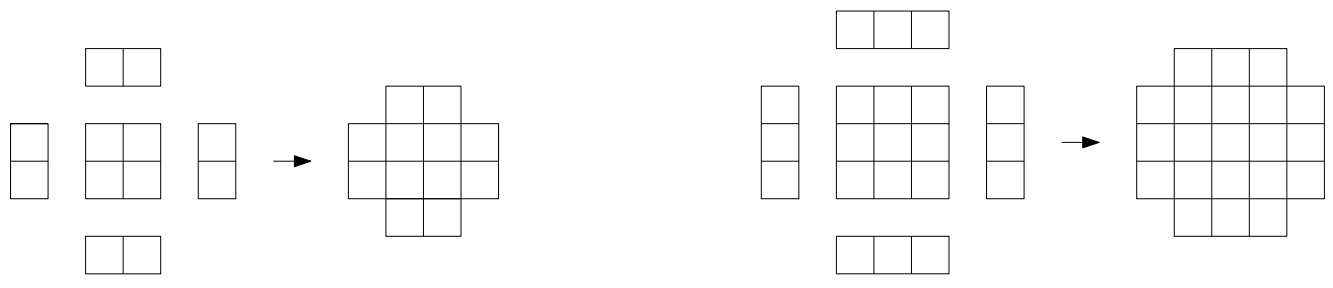

Figure 5.1: construction of X-grids $X_{3}$ and $X_{4}$

\subsection{1 branchwidth of X-grids}

In this section, we study the branchwidth of X-grids. Our findings are encapsulated in the following theorem.

Theorem 5.2.1. For the $X$-grid $X_{n}, b w\left(X_{n}\right)=n$.

Proof. It is well known that the branchwidth of an $n$-grid is equal to $n$. Furthermore, for any minor $H$ of graph $G, b w(H) \leq b w(G)$. Since the $n$-grid is a minor of $X_{n}$ we conclude from the two foregoing observations that $b w\left(X_{n}\right) \geq n$.

To show that $b w\left(X_{n}\right) \leq n$, we construct a branch decomposition of $X_{n}$ of width $n$. To do so, we split the edge set $E$ of $X_{n}$ up in four symmetrical parts $E_{1}, \ldots, E_{4}$, as is done in Figure 5.2 for $X_{4}$. Note that for both even and odd values of $n$, it is easy to make a partition of $E$ in $X_{n}$ consisting of 4 symmetrical parts. 
Ternary tree $T$ of the branch-decomposition consists of one middle edge and four symmetrical subtrees $T_{i}$ that contain the leaves of $T$ corresponding to edges from set $E_{i}$, for $i=1 \ldots 4$. The middle edge of $T$ has order equal to $n$, the edges of $T$ that are incident to a leaf have order 2 and all other edges in $T$ have order at most $n$. The width of the branch decomposition for $X_{n}$ is therefore equal to $n$.

Figure 5.3 illustrates how to construct such an optimal branch decomposition of $X_{4}$. Each edge of $X_{4}$ in Figure 5.2 is denoted by a number. In Figure 5.3, the bijection $\tau$ between the leaves of $T$ and $E$ is displayed by simply putting these numbers in the leaves of $T$. The labels on edges $e$ of $T$ denote the set of vertices $v$ of $X_{4}$ for which there are leaves $t_{1}, t_{2}$ of $T$ in different components of $T \backslash e$, with $\tau\left(t_{1}\right), \tau\left(t_{2}\right)$ both incident to $v$. Following the same idea as is illustrated in Figures 5.2 and 5.3, it is easy to construct a branch decomposition of width $n$ for $X_{n}$ for any value of $n \geq 2$.

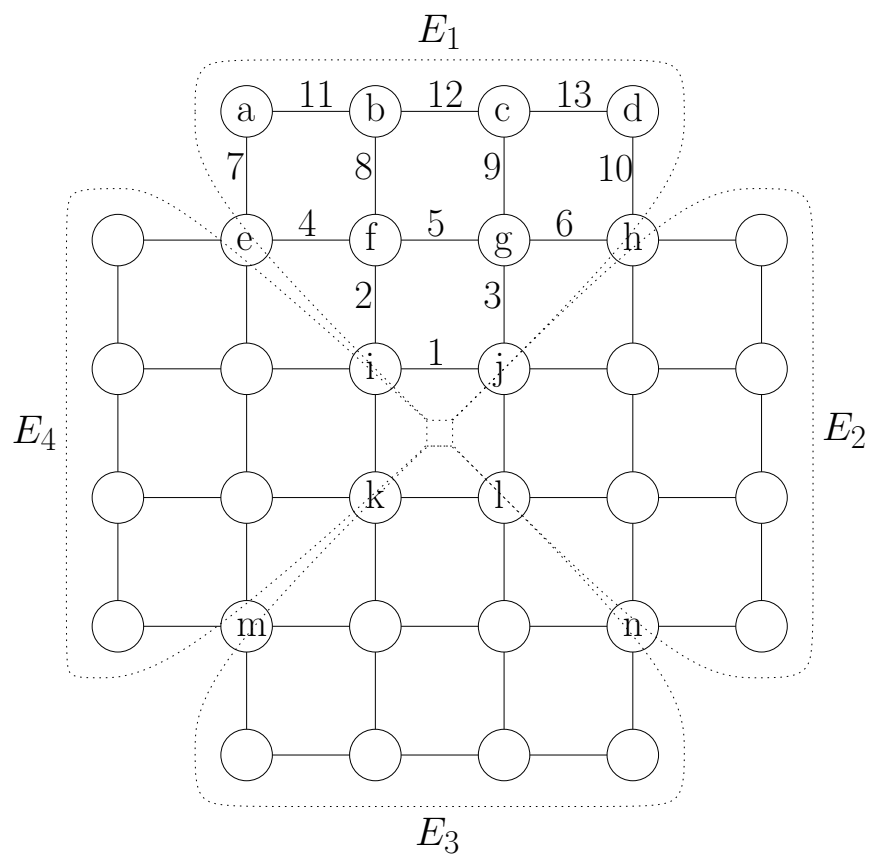

Figure 5.2: partition of $E$ into sets $E_{1}, \ldots, E_{4}$ in planar graph $X_{4}$ 


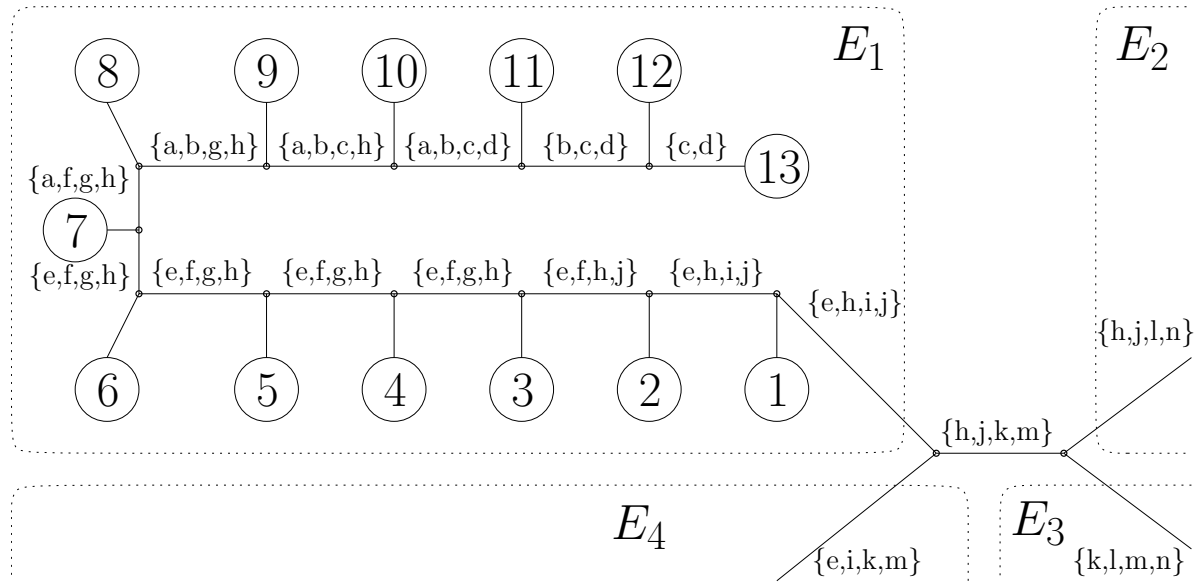

Figure 5.3: an optimal branch decomposition for the graph from Figure 5.2

\subsection{2 treewidth of X-grids}

In this section, we take a closer look at the treewidth of X-grids. The results of this study is condensed in the following theorem.

Theorem 5.2.2. For $X$-grid $X_{n},\left\lceil\frac{3 n}{2}\right\rceil-2 \leq t w\left(X_{n}\right) \leq\left\lfloor\frac{3 n}{2}\right\rfloor-1$.

Proof. It is easy to construct a tree decomposition for $X_{n}$ of width $\left\lfloor\frac{3 n}{2}\right\rfloor-1$. Another way to prove that $t w\left(X_{n}\right) \leq\left\lfloor\frac{3 n}{2}\right\rfloor-1$ is to combine the result from Theorem 5.2.1 with the fact that $t w \leq\left\lfloor\frac{3 b w}{2}\right\rfloor-1$.

To prove that $t w\left(X_{n}\right) \geq\left\lceil\frac{3 n}{2}\right\rceil-2$, we construct a bramble $\mathscr{B}$ of $X_{n}$ of order $\left\lceil\frac{3 n}{2}\right\rceil-1$. From this bramble it follows that the bramble number of $X_{n}$ is at least $\left\lceil\frac{3 n}{2}\right\rceil-1$. Then, we straightforwardly apply Theorem 5.1.5.

For an illustration of the bramble construction, we refer to Figure 5.4(a-b). We split the rows of $X_{n}$ into sets $R_{1}, R_{2}$ and $R_{3}$ and the columns into sets $C_{1}, C_{2}$ and $C_{3}$. The bramble $\mathscr{B}$ now consists of all subsets that are of one of the following four types.

1. The vertices from one row and from one column intersecting this row.

2. The vertices from one column from $C_{1}$, one column from $C_{3}$ and a path between these two columns;

3. The vertices from one row in $R_{3}$, one column in $C_{1}$ and a path between this row and column;

4. The vertices from one row in $R_{3}$, one column in $C_{3}$ and a path between this row and column. 
One subset of $\mathscr{B}$ of type 1 and one subset of type 2 are depicted in Figure 5.4(a) with respectively fat solid lines and fat dashed lines. Subsets of $\mathscr{B}$ of type 3 and 4 are depicted respectively by the fat solid lines and the fat dashed lines in Figure 5.4(b). It can be easily
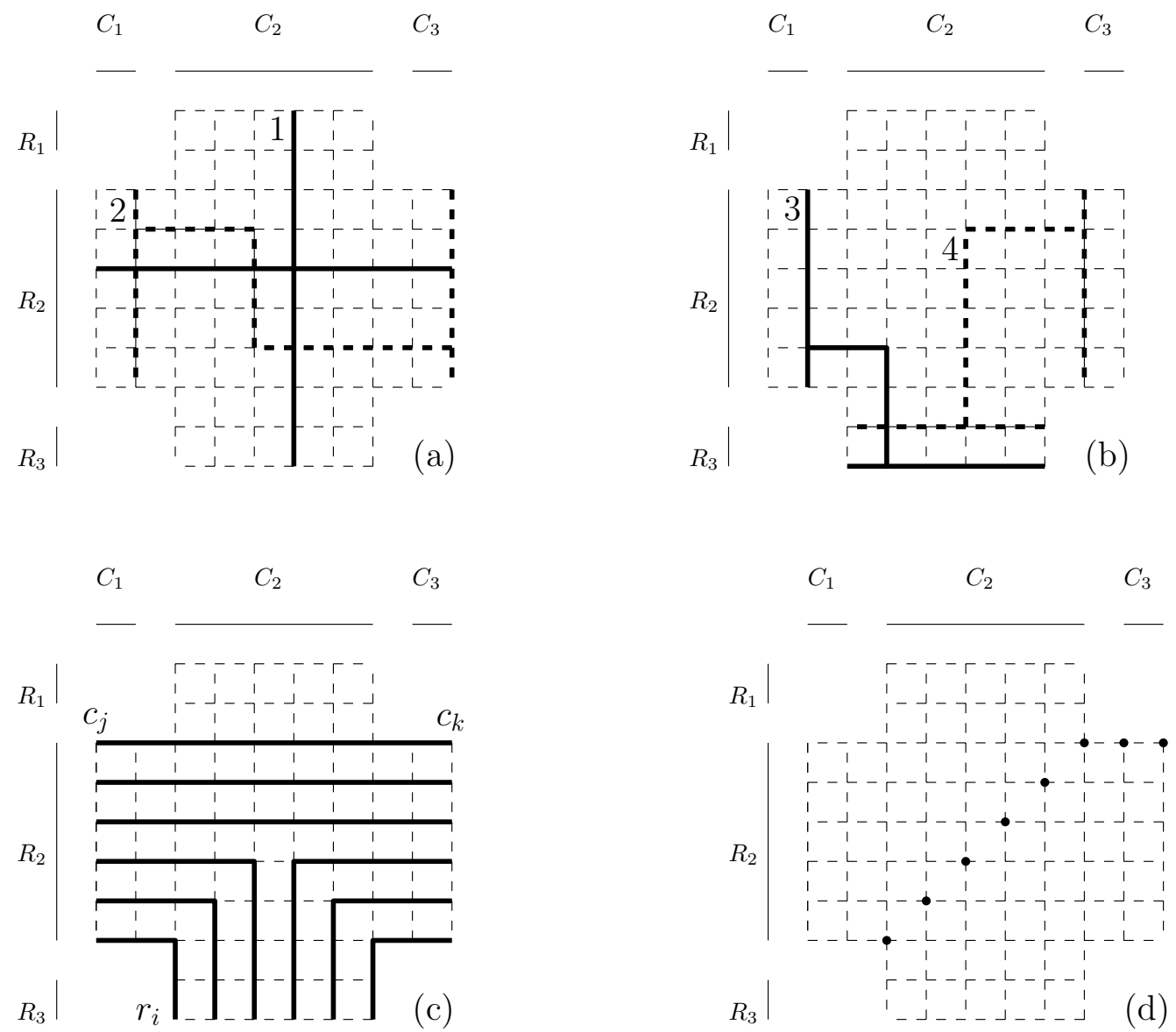

Figure 5.4: Examples of the four types of subsets of $\mathscr{B}$ in (a,b), a collection of $\left\lfloor\frac{3 n}{2}\right\rfloor$ vertex disjoint paths in (c) and a hitting set for $\mathscr{B}$ of size $\left\lceil\frac{3 n}{2}\right\rceil-1$ in (d).

verified that the subsets of $\mathscr{B}$ are connected and mutually touching. We will now show that the order of $\mathscr{B}$ is equal to $\left\lceil\frac{3 n}{2}\right\rceil-1$ which will thus imply that $t w\left(X_{n}\right) \geq\left\lceil\frac{3 n}{2}\right\rceil-2$.

First we prove that the order of $\mathscr{B}$ is at least $\left\lceil\frac{3 n}{2}\right\rceil-1$ by showing that for every vertex set $V^{\prime}$ such that $\left|V^{\prime}\right|<\left\lceil\frac{3 n}{2}\right\rceil-1$, there is a subset of $\mathscr{B}$ that is not hit by $V^{\prime}$. Consider such a set $V^{\prime}$. Note that $R_{2}$ and $R_{3}$ together have $\left\lceil\frac{3 n}{2}\right\rceil-1$ rows and $C_{1}, C_{2}$ and $C_{3}$ have more than $\left\lceil\frac{3 n}{2}\right\rceil-1$ columns together. Thus, if every row in $R_{2}$ is hit by $V^{\prime}$, then there is a row in $R_{3}$ that is not hit by $V^{\prime}$. Similarly, if every column in $C_{2}$ is hit by $V^{\prime}$, then there is a column in 
$C_{1}$ and a column in $C_{3}$ that are not hit by $V^{\prime}$. Therefore, for any $V^{\prime}$ with $\left|V^{\prime}\right|<\left\lceil\frac{3 n}{2}\right\rceil-1$, at least one of the following 4 situations occurs:

S1. Row $r_{i}$ from $R_{2}$ and column $c_{j}$ from $C_{2}$ are not hit by $V^{\prime}$.

S2. Row $r_{i}$ from $R_{2}$ and column $c_{j}$ from $C_{1}$ are not hit by $V^{\prime}$.

S3. Row $r_{i}$ from $R_{3}$ and column $c_{j}$ from $C_{2}$ are not hit by $V^{\prime}$.

S4. Row $r_{i}$ from $R_{3}$, column $c_{j}$ from $C_{1}$ and column $c_{k}$ from $C_{3}$ are not hit by $V^{\prime}$.

We show that in each of these 4 situations, there is a subset of $\mathscr{B}$ that is not hit by $V^{\prime}$. In situation $\mathrm{S} 1, \mathrm{~S} 2$ and $\mathrm{S} 3, r_{i}$ and $c_{j}$ define a subset of $\mathscr{B}$ of type 1 . Situation $\mathrm{S} 4$ needs somewhat more attention. First we point out that any vertex set in $X_{n}$ that separates $r_{i}$ from $c_{j}$ and from $c_{k}$ and simultaneously separates $c_{j}$ from $c_{k}$ contains at least $\left\lfloor\frac{3 n}{2}\right\rfloor$ vertices. See Figure 5.4(c), where $\left\lfloor\frac{3 n}{2}\right\rfloor$ vertex disjoint paths between $r_{i}, c_{j}$ and $c_{k}$ are depicted. Since $V^{\prime}$ contains strictly less than $\left\lceil\frac{3 n}{2}\right\rceil-1$ vertices and thus strictly less than $\left\lfloor\frac{3 n}{2}\right\rfloor$ vertices, in situation S4 there exists thus a path $P$ either between $c_{j}$ and $c_{k}$ or between $r_{i}$ and $c_{j}$ or between $r_{i}$ and $c_{k}$ such that $P$ is not hit by $V^{\prime}$. In the first case, $\left\{c_{j}, P, c_{k}\right\}$ forms a subset of $\mathscr{B}$ of type 2 that is not hit by $V^{\prime}$. In the second case $\left\{r_{i}, P, c_{j}\right\}$ forms a subset of type 3 of $\mathscr{B}$ that is not hit by $V^{\prime}$ and in the last case $\left\{r_{i}, P, c_{k}\right\}$ forms a subset of $\mathscr{B}$ of type 4 that is not hit by $V^{\prime}$. This shows that any set $V^{\prime}$ of size strictly smaller than $\left\lceil\frac{3 n}{2}\right\rceil-1$ can not be a hitting set of bramble $\mathscr{B}$. Hence the order of $\mathscr{B}$ is at least $\left\lceil\frac{3 n}{2}\right\rceil-1$.

To show that the order of $\mathscr{B}$ is equal to $\left\lceil\frac{3 n}{2}\right\rceil-1$, we note that the vertex set $S$ as depicted in Figure $5.4(\mathrm{~d})$ forms a hitting set of $\mathscr{B}$ of size $\left\lceil\frac{3 n}{2}\right\rceil-1$. It is easy to verify that all four types of subsets in $\mathscr{B}$ are indeed hit by this hitting set.

\subsection{3 largest square-grid minor of X-grids}

By construction, $X_{n}$ contains an $n$-grid minor as an induced subgraph. Intuitively, it might seem clear that the side size of the largest square-grid minor in $X_{n}$ is equal to $n$. Next, we present two proofs that support this intuition. The first proof is an easy one and is based on results concerning branch decompositions. Without the use of branch decompositions however, finding a proof turned out to be less trivial than we thought. In our second proof, we do not resort to branch decompositions. Instead, we use arguments related to face distances in the graph.

Theorem 5.2.3. Given $X$-grid $X_{n}, \operatorname{gm}\left(X_{n}\right)=n$.

Proof. When we combine the result of Theorem 5.2.1 with the fact that for any graph $G$ it holds that $g m(G) \leq b w(G)$, we find that $g m\left(X_{n}\right) \leq n$. By construction, $X_{n}$ contains an $n$ grid as an induced subgraph, from which we conclude that $\operatorname{gm}\left(X_{n}\right) \geq n$. The two foregoing observations yield that $g m\left(X_{n}\right)=n$. 
Surprisingly enough, without the use of branchwidth it is rather difficult to prove Theorem 5.2.3. In the alternative proof that will be presented next, we use arguments in terms of face distances as they are defined in Section 5.1. This proof will provide a methodological technique in contrast to the branchwidth based proof.

Before we start with the alternative proof, we need several propositions. The propositions concern a mapping of the face set of a planar embedding of a graph $G$ to the face set of a planar embedding of a minor $M$ of $G$. We recall that a minor $M$ can be obtained from $G$ by a series of vertex deletions, edge deletions and edge contractions. Basically, if such operation does not change the number of faces in the embedding, then a face is mapped to the face in the embedding of the minor that naturally corresponds to it. If, by a vertex deletion or edge deletion, some faces are joined together to one face, then all these faces are mapped to the joined face. If, by an edge contraction, some face $f$ disappears, then $f$ is mapped to a face in the embedding of the minor that naturally corresponds to a neighbor face of $f$. We say that face $f^{\prime}$ in an embedding of minor $M$ of $G$ corresponds to face $f$ in an embedding of $G$ if $f$ can be mapped to $f^{\prime}$. We now introduce three propositions, for which correctness can be easily verified.

Proposition 5.2.4. Using the mapping described above, each face in an embedding of $G$ can be mapped to a face in an embedding of a minor $M$ of $G$. Moreover, for any number $i$ of different faces in the embedding of $M$, there are at least $i$ different faces in the embedding of $G$ that can respectively be mapped to them.

Proposition 5.2.5. Let $f^{\prime}$ and $f^{\prime \prime}$ be two faces in the embedding of $G$ and let $f_{M}^{\prime}$ and $f_{M}^{\prime \prime}$ be respectively the two faces corresponding to $f^{\prime}$ and $f^{\prime \prime}$ in the embedding of a minor $M$ of $G$. Then, $d_{M}\left(f_{M}^{\prime}, f_{M}^{\prime \prime}\right) \leq d_{G}\left(f^{\prime}, f^{\prime \prime}\right)$.

Proposition 5.2.6. Let $f^{\prime}$ be a face and $v$ be a vertex in $G$ and let $M$ be a minor of $G$ in which $v$ is not deleted nor contracted to another vertex. Furthermore, let $f_{M}^{\prime}$ be a face in $M$ corresponding to $f^{\prime}$. Then, $d_{M}\left(v, f_{M}^{\prime}\right) \leq d_{G}\left(v, f^{\prime}\right)$.

Using these propositions, we are now ready to present an alternative proof of Theorem 5.2.3.

Proof. Again by construction of $X_{n}$, it is easy to see that $X_{n}$ contains an $n$-grid as a minor. To show that $g m\left(X_{n}\right)=n$, we show that $X_{n}$ has no $(n+1)$-grid minor. We will show that the outer face in an embedding of $X_{n}$ cannot be mapped to any face in an embedding of the $(n+1)$-grid. Then by Proposition 5.2.4 we derive that the $(n+1)$-grid cannot be a minor of $X_{n}$. The proof is divided into three cases. For all three cases, we present arguments only for even values of $n$. For odd values of $n$ the proof is similar. We consider the natural embedding of $X_{n}$ and of the $(n+1)$-grid and from here on, we just talk about a face of $X_{n}((n+1)$-grid) instead of about a face in the natural embedding of $X_{n}((n+1)$-grid). 
Case 1. The outer face of $X_{n}$ cannot be mapped to the outer face of the $(n+1)$-grid. Because $n$ is even, there is a vertex $v$ in the $(n+1)$-grid that has distance $\frac{n}{2}$ to the outer face $f^{\prime}$, see the leftmost picture in Figure 5.5. If the outer face of $X_{n}$ could be mapped to the outer face of the $(n+1)$-grid, then by Proposition 5.2.6 there should also be a vertex in $X_{n}$ with distance at least $\frac{n}{2}$ to the outer face of $X_{n}$. However, there are no such vertices in $X_{n}$, see the rightmost picture in Figure 5.5.
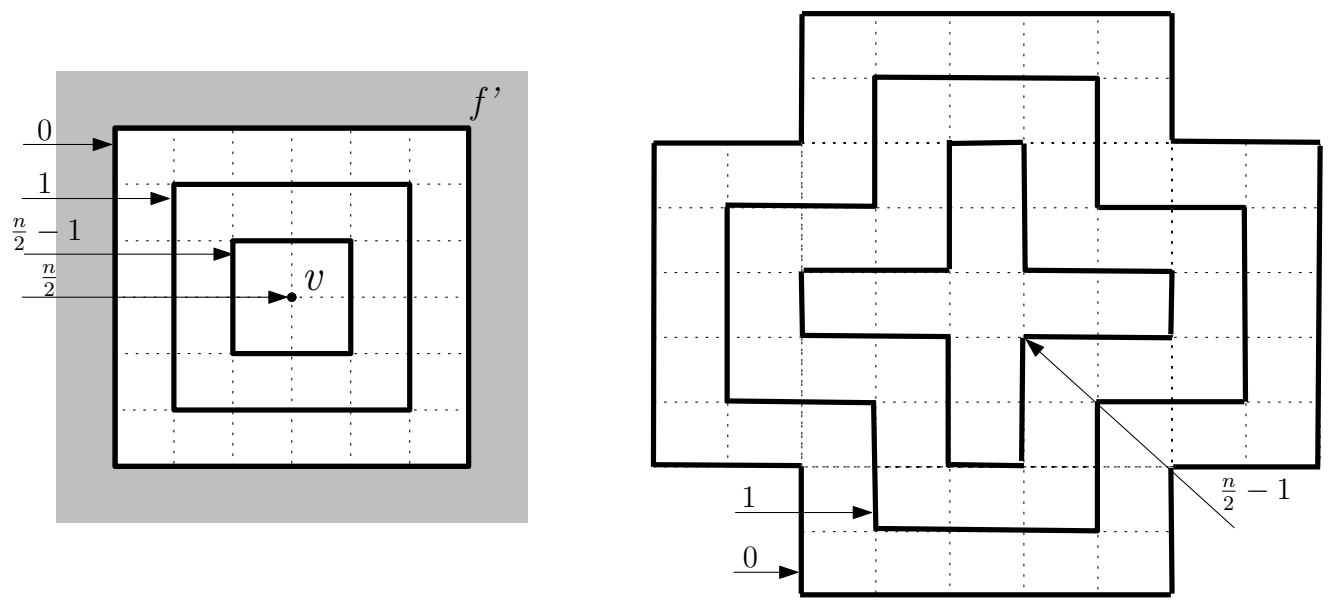

Figure 5.5: Distances of vertices to the outer face in the $(n+1)$-grid and in $X_{n}$

Case 2. We now show that the outer face of $X_{n}$ cannot be mapped to any of the 4 middle faces of the $(n+1)$-grid. There are $2 n$ faces in the $(n+1)$-grid having face distance $\frac{n}{2}$ to a middle face $f^{\prime}$, see Figure 5.6, leftmost picture. Suppose that the outer face of $X_{n}$ can be mapped to any of the 4 middle faces of the $(n+1)$-grid. By Propositions 5.2.4 and 5.2.5 then there must be $2 n$ different faces in $X_{n}$ at face distance at least $\frac{n}{2}$ to the outer face. However, there are only $2 n-3$ such faces in $X_{n}$, see the rightmost picture in Figure 5.6.

Case 3. Finally, let us show that the outer face of $X_{n}$ cannot be mapped to any of the other inner faces of the $(n+1)$-grid. For each such inner face of the $(n+1)$-grid there is a vertex at distance $\frac{n}{2}$, see Figure 5.7. Suppose that the outer face of $X_{n}$ can be mapped to one of the other inner faces of the $(n+1)$-grid. Then, by Proposition 5.2.6 there must be a vertex in $X_{n}$ that has distance at least $\frac{n}{2}$ to the outer face. Again, there is no such vertex in $X_{n}$, see the rightmost picture in Figure 5.5. 

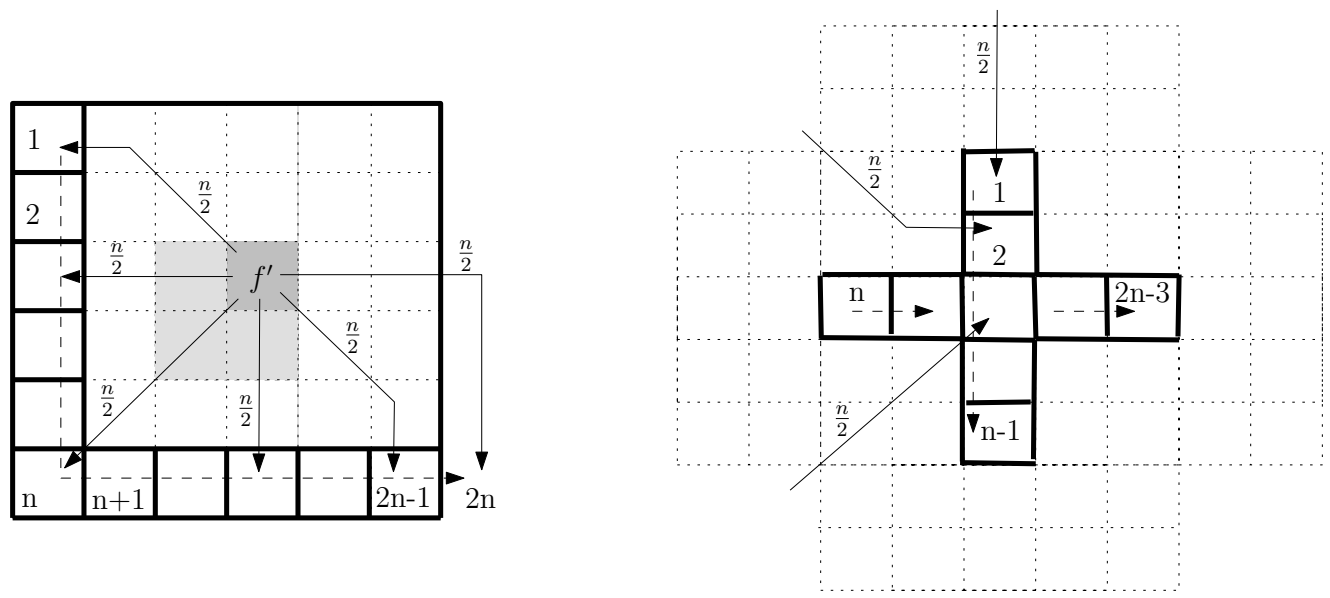

Figure 5.6: There are $2 n$ faces at face distance $\frac{n}{2}$ to $f^{\prime}$ in the $(n+1)$-grid and $2 n-3$ faces at face distance $\frac{n}{2}$ to the outer face in $X_{n}$

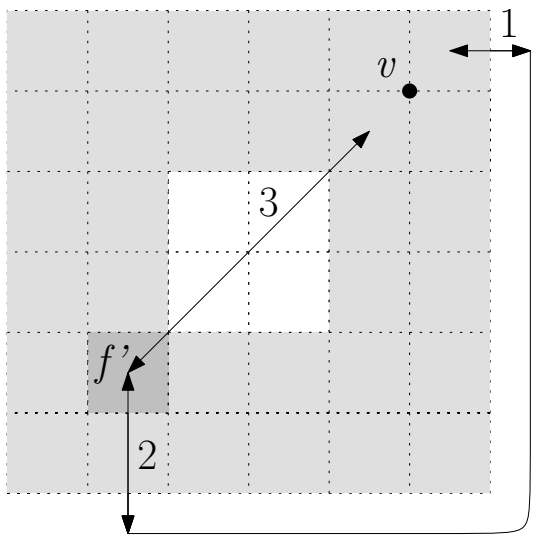

Figure 5.7: Vertex $v$ at distance $\frac{n}{2}$ to a non-middle inner face $f^{\prime}$ in the $(n+1)$-grid

\subsection{Sandwich grids and pyramids}

In this section, we introduce two more classes of planar graphs, which we call pyramids and sandwich grids. The graphs in the pyramid family will be referred to as $\Lambda_{n}$, whereas $S_{n}$ will be used to denote a sandwich grid. The pyramid $\Lambda_{n}$ is a graph on $2 n^{2}-2 n+1$ vertices and $6 n^{2}-10 n+4$ edges. It can be constructed by building a pyramid with the side size of the base level equal to $n$, as shown in the leftmost picture in Figure 5.8. The sandwich-grid $S_{n}$ is a graph on $2 n^{2}$ vertices and $4 n^{2}-4$ edges and can be constructed by taking two $n$-grids and 
connecting the vertices on the outer face of one $n$-grid to the corresponding vertices on the outer face of the second $n$-grid, as is shown in the rightmost picture in Figure 5.8. Planar embeddings of the graphs $\Lambda_{3}$ and $S_{4}$ are given in Figure 5.9.
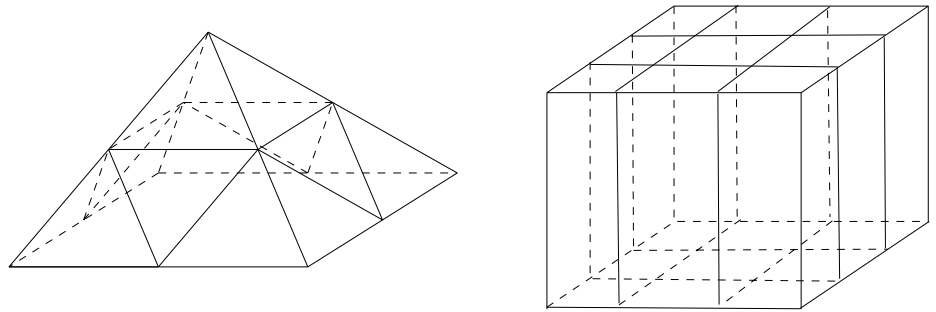

Figure 5.8: pyramid $\Lambda_{3}$ and sandwich grid $S_{4}$
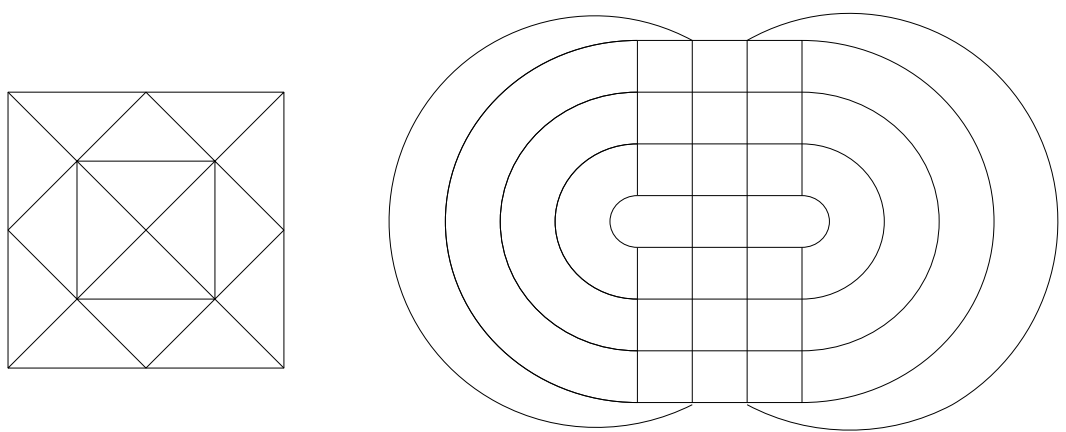

Figure 5.9: planar embeddings for pyramid $\Lambda_{3}$ and sandwich grid $S_{4}$

In the following we will determine the treewidth and branchwidth of sandwich grids. We will also present a lower bound on the side size of the largest square-grid minor for both families and an upper bound on the treewidth of pyramids. We conjecture that these bounds are tight.

\subsection{1 branchwidth of sandwich grids}

The branchwidth of sandwich grids can be roughly determined, but only indirectly, as is shown in the proof of the following theorem.

Theorem 5.3.1. For a sandwich grid $S_{n}, 2 n \leq b w\left(S_{n}\right) \leq 2 n+1$. 
Proof. To show that $b w\left(S_{n}\right) \leq 2 n+1$, we use the result from the next section that $t w\left(S_{n}\right) \leq$ $2 n$. When we combine this with the fact that for any graph $G$ it holds that $b w(G) \leq t w(G)+1$, we obtain the desired result.

To show that $b w\left(S_{n}\right) \geq 2 n$, we use a result on cylinder graphs. Gu and Tamaki show in (70) that for cylinder $C_{2 n, n}$, which is a subgraph of $S_{n}, b w\left(C_{2 n, n}\right)=2 n$. Since the branchwidth of a subgraph of $S_{n}$ is smaller than or equal to $b w\left(S_{n}\right)$, we conclude that $b w\left(S_{n}\right) \geq$ $2 n$.

The question to determine the branchwidth of the pyramid class $\Lambda_{n}$ still remains open. Later we will show that $t w\left(\Lambda_{n}\right) \leq 2 n-1$. Using this result, we can bound $b w\left(\Lambda_{n}\right)$ from above by $2 n$.

\subsection{2 treewidth of sandwich grids and pyramids}

The treewidth of sandwich grids can be approximated as follows.

Theorem 5.3.2. For sandwich grid $S_{n}, 2 n-1 \leq t w\left(S_{n}\right) \leq 2 n$.

Proof. To prove that $t w\left(S_{n}\right) \leq 2 n$, we show how to construct a tree decomposition (actually, a path decomposition) of $S_{n}$ of width $2 n$. One can start with a bag that contains $2 n$ vertices from the first column of $S_{n}$ (using the embedding of Figure 5.9) plus the top vertex of the second column. In the next bag, we eliminate the top vertex from the first column and introduce the second vertex from the second column, etc. The last bag contains the bottom vertex of the one-to-last column and the $2 n$ vertices from the last column in $S_{n}$.

To show that $t w\left(S_{n}\right) \geq 2 n-1$, we use a result from (70). A result from this study is that for subgraph $C_{2 n, n}$ of $S_{n}$ it holds that $b w\left(C_{2 n, n}\right)=2 n$. Therefore $b w\left(S_{n}\right) \geq 2 n$. Combined with the fact that $b w\left(S_{n}\right) \leq t w\left(S_{n}\right)+1$ this implies that $t w\left(S_{n}\right) \geq 2 n-1$.

For the pyramid $\Lambda_{n}$, we construct a tree decomposition showing that $t w\left(\Lambda_{n}\right) \leq 2 n-1$. Consider one of the main diagonals in $\Lambda_{n}$ (using the embedding from Figure 5.9) and all other paths in $\Lambda_{n}$ that are parallel to this diagonal. Let the middle bag of the tree decomposition contain all vertices from the main diagonal. In both directions we add a bag containing the vertices from the main diagonal plus the top vertex from the next path. After that, we simply eliminate the vertices one by one. This will give a path decomposition of $\Lambda_{n}$ of width $2 n-1$, hence $t w\left(\Lambda_{n}\right) \leq 2 n-1$. The question to prove tightness of this upper bound is still open at the moment of writing this thesis.

\subsection{3 square-grid minor of sandwich grids and pyramids}

It is easy to see that $\operatorname{gm}\left(S_{n}\right) \geq n$ since $S_{n}$ contains the $n$-grid as an induced subgraph. To show that $\operatorname{g} m\left(\Lambda_{n}\right) \geq n$, we refer to Figure 5.10, which illustrates how to obtain an $n$-grid 
minor in $\Lambda_{n}$ for odd and even values of $n$. We do believe that both families $S_{n}$ and $\Lambda_{n}$ do not contain $(n+1)$-grid minors, but we are still looking for techniques to prove this.

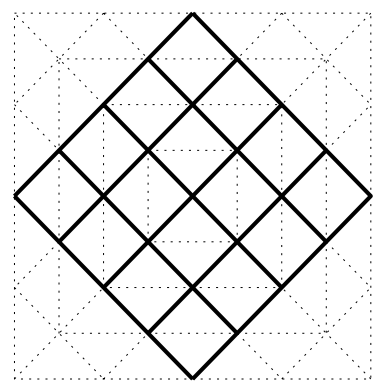

odd $n$

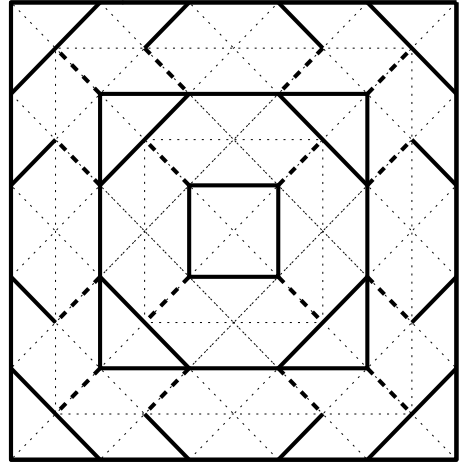

even $n$

Figure 5.10: To obtain $n$-grid minors in $\Lambda_{n}$, delete dotted edges and vertices that are incident only to dotted edges and contract the dashed edges.

\subsection{Summary and open questions}

The results from this study are summarized in Table 5.1. As a direction for further research,

\begin{tabular}{|l|c|c|c|}
\hline graph family & gm & bw & tw \\
\hline X-grid $\left(X_{n}\right)$ & $n$ & $n$ & $\approx \frac{3 n}{2}-1$ \\
\hline pyramid $\left(\Lambda_{n}\right)$ & $\begin{array}{c}\geq n \\
\leq 2 n\end{array}$ & $\begin{array}{c}\geq n \\
\leq 2 n\end{array}$ & $\begin{array}{c}\geq n \\
\leq 2 n-1\end{array}$ \\
\hline sandwich grid $\left(S_{n}\right)$ & $\begin{array}{c}\geq n \\
\leq 2 n\end{array}$ & $\approx 2 n$ & $\approx 2 n-1$ \\
\hline
\end{tabular}

Table 5.1: summary of results

we recommend the interested reader to consider the following questions.

1. Can we find a technique to prove that $\operatorname{gm}\left(\Lambda_{n}\right), \operatorname{gm}\left(S_{n}\right) \leq n$ ?

2. Can we find a bramble for $\Lambda_{n}$ of order $2 n$, i.e., can we show that $t w\left(\Lambda_{n}\right)=2 n-1$ ? 
3. Can we find a family of planar graphs satisfying $c_{1} g m<b w<c_{2} t w$, where $c_{1}>1$ and $c_{2}<1$ are some constants?

We end this chapter with the conjecture that the sandwich grid and the pyramid are worst cases for branchwidth and treewidth approximation in terms of the side size of the largest square-grid minor.

Conjecture 5.4.1. For any planar graph $G$, both $b w(G)$ and $t w(G)$ are at most $2 g m(G)+$ $o(g m(G))$. 



\section{Chapter 6}

\section{$H$-Subgraph Edge Deletion}

In the final chapter of this thesis, we shift our focus to an application of treewidth and tree decompositions. We demonstrate how an otherwise intractable graph theoretical problem can be solved in linear time on graphs that have bounded treewidth, if a tree decomposition of the graph is available.

In the field of combinatorial graph theory, lately there has been an increased interest in algorithms for graphs not containing some specified subgraph or induced subgraph. An apparent reason for this interest is that many combinatorial problems on graphs have useful structural and/or algorithmic properties when restricted to graphs that exclude certain subgraphs. Just to give a few insightful examples: a famous result by Erdös, Kleitman and Rothschild (59) says that almost every triangle-free graph has chromatic number 2; Minty (92) and Sbihi (99) show that the maximum independent set problem is polynomially solvable on claw-free graphs; Dunbar and Frick (58) show that the path-partition conjecture is true for claw-free graphs; Borodin et al. (40) prove that planar graphs with no cycles of length from 4 to 7 are 3-colorable. There are, of course, many other structural and algorithmic results related to graphs without (a) specific subgraph(s). Motivated by this increased attention in the literature, we present algorithms in this chapter to turn a graph into a graph that excludes certain subgraphs by deleting a minimum number of edges from it.

The chapter is organized as follows. In Section 6.1 we introduce the problem that will be the subject of study in this chapter and we give some references to related studies. We continue by showing in Section 6.2 that by a general result of Courcelle, the decision version of the considered problem is theoretically solvable in linear time on graphs of bounded treewidth. We then introduce a dynamic program in Section 6.4 for the case where the input graph has bounded maximum degree that solves the problem in linear time on graphs of bounded treewidth. In Section 6.4 the subgraph that is excluded from the graph can be any fixed, connected graph. Subsequently in Section 6.5, we consider the case where the fixed subgraph forms a clique and we present a dynamic program that solves the problem 
in linear time on graphs of bounded treewidth. In Section 6.6, we present Baker's style approximation schemes for the problems from Sections 6.4 and 6.5 on planar graphs. Finally, in Section 6.7, we show how our algorithms can be adopted to deal with the situation where more than one type of subgraph needs to be excluded from the input graph.

The content of this chapter is based on cooperation with Alexander Grigoriev and Natalya Usotskaya.

\subsection{Problem definition}

We consider the graph theoretical problem of turning a graph into a graph that excludes certain subgraphs. The transformation is executed by removing edges from the input graph. Given an input graph $G$ and a finite set of graphs $\mathscr{H}=\left\{H_{1}, \ldots, H_{t}\right\}$, we call a subgraph of $G$ an $H$-subgraph of $G$ if it is isomorphic to one of the graphs in the set $\mathscr{H}$. In this context, a graph $G$ is usually referred to as the text and $\mathscr{H}$ as the set of patterns. A graph $G$ is called $H$-free if there are no $H$-subgraphs in $G$. For ease of notation, we use notions of $H$-subgraphs and $H$-free graphs instead of $\mathscr{H}$-subgraphs and $\mathscr{H}$-free graphs.

We restrict ourselves to the case in which the subgraphs that have to be excluded are fixed graphs that are connected. From here on, we will therefore assume that $\mathscr{H}$ forms a finite set of fixed, connected patterns $H_{i}$. The optimization problem that we consider in this chapter is the following.

PROBLEM: Minimum $H$-Subgraph Edge Deletion

Input: $\quad$ Text graph $G$ and finite set of patterns $\mathscr{H}=\left\{H_{1}, \ldots, H_{t}\right\}$

Question: What is the minimum number of edges that must be deleted from $G$ to make it $H$-free?

We refer to the decision version of this problem as $H$-SUbGRAPH EdGe Deletion; i.e., given integer $k$, is it possible to make the input graph $H$-free by deleting at most $k$ edges?

Related work. It is well-known that the TRIANGLE EDGE DELETION problem, a special case of $H$-Subgraph EdGe Deletion with $\mathscr{H}=\left\{K_{3}\right\}$, is $N P$-complete, see Yannakakis (113). Therefore the problem $H$-Subgraph Edge DELETion is $N P$-complete as well. Recently, Brügmann et al. (48) proved that the problem TRIANGLE EdGe DELETION remains $N P$-complete even if the graph is planar and has maximum degree of 7 . On the positive side, they construct polynomial time reduction rules to obtain linear problem kernels.

Closely related problems are considered extensively in the extremal graph theory; see, e.g., Bollobás (38) and Bohman (37). There, the general question is: Given a graph $H$ and a number $n$, what is the maximum number of edges in a graph $G$ on $n$ vertices that does not 
contain a subgraph isomorphic to $H$ ? In the context of this paper, the bounds obtained in the extremal graph theory can be seen as the source of generic lower bounds on the number of edges to be removed to make a given graph $H$-free.

To construct PTASs for Minimum $H$-SUbGRAPH EdGE DELETION on planar graphs, we employ the layerwise decomposition approach introduced by Baker (11). A generalization of this approach that can be applied also to problems with a non-local structure has recently been developed by Demaine and Hajiaghayi and is an extension of the bidimensionality theory. For a literature overview on bidimensionality and its connections to approximation schemes for planar graph problems, we refer to (55) and (54).

Our results. In this chapter, we first implicitly present a linear time algorithm for $H$ Subgraph Edge Deletion on graphs of bounded treewidth, based on a framework by Courcelle $(51 ; 52)$. We then introduce constructive linear time algorithms for MINIMUM $H$-Subgraph Edge Deletion for the two cases for which respectively $G$ has bounded maximum vertex degree and $H$ is a clique. Both these algorithms are dynamic programs that assume as input a tree decomposition of the input graph. Finally, for the same cases we design PTASs for Minimum $H$-SUbGRAPH EdGE DELETION on planar graphs.

\subsection{MSOL Formulations}

Bounded treewidth and Monadic Second Order Logic (MSOL) have proven to be key concepts in establishing fixed-parameter tractability results. The general results of Courcelle and Arnborg et al (9) provide a host of powerful algorithmic tools for many combinatorial problems on graphs of bounded treewidth. In particular, they show that any property on graph $G$ that can be expressed in MSOL, can be decided in linear time if $G$ has bounded treewidth.

\subsection{1 formulation for single pattern}

Consider the $H$-SUbGRAPH EdGe DELETION problem where $\mathscr{H}$ consists of a single pattern $H$. By encoding this problem in MSOL, we implicitly construct a linear time algorithm solving $H$-SubGRAPH EdGE DELETION on graphs of bounded treewidth. We end the section by generalizing the results to allow for a finite set $\mathscr{H}$ of patterns instead of just a single pattern.

Consider the following relational structure:

$$
\mathbf{G}=\left\{V(G), E(G), V(H), E(H), R_{G}^{2}, R_{H}^{2}, E q^{2}, f^{1}\right\},
$$

where 
- $R_{G}(x, e)$ and $R_{H}(x, e)$ are the vertex-edge incidence relation in $G$ and $H$ respectively;

- $E q(x, y)$ is the equality predicate;

- $f: S \rightarrow V(H)$ is a function with domain $S \subseteq V(G)$ and image $V(H)$.

We now describe a formula $\Phi(k, G, H)$ such that $\mathbf{G}=\Phi(k, G, H)$ if and only if there exists a set of edges $F \subseteq E(G)$ of cardinality at most $k$ covering all $H$-subgraphs of the graph $G$.

Let $G^{\prime}$ be a subgraph of $G$. First, we define the formula $\Psi\left(f, G^{\prime}\right)$ to express that $f$ is an isomorphism between $G^{\prime}$ and $H$. The following properties should be satisfied:

- Function $f$ is injective:

$$
\operatorname{Inj}\left(f, G^{\prime}\right)=\forall u, v \in V\left(G^{\prime}\right), E q(f(u), f(v)) \Rightarrow E q(u, v) ;
$$

- Function $f$ is surjective:

$$
\operatorname{Surj}\left(f, G^{\prime}\right)=\forall v \in V(H) \exists u \in V\left(G^{\prime}\right), E q(f(u), v) ;
$$

- Function $f$ preserves the edge relations from $G^{\prime}$ in $H$ :

$$
\begin{gathered}
\overrightarrow{E d g e}\left(f, G^{\prime}\right)=\forall u, v \in V\left(G^{\prime}\right), e \in E\left(G^{\prime}\right), R_{G}(u, e) \& R_{G}(v, e) \Rightarrow \\
\exists e^{\prime} \in E(H), R_{H}\left(f(u), e^{\prime}\right) \& R_{H}\left(f(v), e^{\prime}\right) ;
\end{gathered}
$$

- Function $f$ preserves the edge relation from $H$ in $G^{\prime}$ :

$$
\begin{gathered}
\overleftarrow{E d g e}\left(f, G^{\prime}\right)=\forall u, v \in V(H), e \in E(H), R_{H}(u, e) \& R_{H}(v, e) \Rightarrow \\
\exists u^{\prime}, v^{\prime} \in V\left(G^{\prime}\right), e^{\prime} \in E\left(G^{\prime}\right), R_{G}\left(u^{\prime}, e^{\prime}\right) \& R_{G}\left(v^{\prime}, e^{\prime}\right) \& E q\left(f\left(u^{\prime}\right), u\right) \& E q\left(f\left(v^{\prime}\right), v\right) .
\end{gathered}
$$

Let

$$
\Psi\left(f, G^{\prime}\right)=\operatorname{Inj}\left(f, G^{\prime}\right) \& \operatorname{Surj}\left(f, G^{\prime}\right) \& \overleftrightarrow{\mathrm{Edge}}\left(f, G^{\prime}\right) \& \overleftrightarrow{\operatorname{Edge}}\left(f, G^{\prime}\right)
$$

and consequently

$$
\begin{gathered}
\Phi(k, G, H)=\exists F \subseteq E(G),|F| \leq k \& \\
\left(\forall V^{\prime} \subseteq V(G), E^{\prime} \subseteq E(G), \Psi\left(f,\left(V^{\prime}, E^{\prime}\right)\right) \Rightarrow\left|F \cap E^{\prime}\right| \geq 1\right) .
\end{gathered}
$$

Hence, we expressed the problem $H$-SubGraph EdGe Deletion in MSOL, which in combination with Courcelle's Theorem proves the following theorem.

Theorem 6.2.1. Problem $H$-Subgraph Edge Deletion with $\mathscr{H}$ consisting of a single fixed, connected pattern $H$ can be solved in $O(n \cdot g(w, h))$ time for some function $g$, where $w$ is the treewidth of $G$ and $h$ is the size (number of vertices) of $H$.

Using the result of Theorem 6.2.1 in combination with binary search, the corresponding optimization version of the problem can be solved in $\mathrm{O}(n \log (m) \cdot g(w, h))$ time, where $m$ denotes the number of edges in $G$. 


\subsection{2 formulation for set of patterns}

To generalize Theorem 6.2.1 to a finite set of patterns $\mathscr{H}=\left\{H_{1}, \ldots, H_{t}\right\}$, we simply need a wider relational structure $\mathbf{G}$ :

$$
\mathbf{G}=\left\{V(G), E(G), V\left(H_{1}\right), E\left(H_{1}\right), \ldots, V\left(H_{t}\right), E\left(H_{t}\right), R_{G}, R_{1}, \ldots, R_{t}, E q, f_{1}, \ldots, f_{t}\right\},
$$

where in addition to the definitions above we have to specify

- $R_{i}(x, e), i=1 \ldots t$, are the vertex-edge incidence relations in $H_{i}$;

- $f_{i}: S \subseteq V(G) \rightarrow V\left(H_{i}\right), i=1 \ldots t$, are functions with domain $S \subseteq V(G)$ and images $V\left(H_{i}\right)$.

Then, we can write down the general formula $\Phi(k, G, \not{H})$ in the following way:

$$
\begin{gathered}
\Phi(k, G, \not{H})=\exists F \subseteq E(G),|F| \leq k \& \\
\left(\forall V^{\prime} \subseteq V(G), E^{\prime} \subseteq E(G), \exists 1 \leq i \leq t, \Psi\left(f_{i},\left(V^{\prime}, E^{\prime}\right)\right) \Rightarrow\left|F \cap E^{\prime}\right| \geq 1\right) .
\end{gathered}
$$

This MSOL formulation, in combination with Courcelle's Theorem, proves the following theorem.

Theorem 6.2.2. Problem $H$-Subgraph EdGe Deletion with a finite set $\mathscr{H}=\left\{H_{1}, \ldots, H_{t}\right\}$ of fixed, connected patterns can be solved in $O(n \cdot g(w, h))$ time for some function $g$, where $w$ is the treewidth of $G$ and $h$ is the size of the largest pattern in $\mathscr{H}$.

To solve the corresponding optimization version of the problem, one can combine Theorem 6.2.2 with binary search, which adds a factor $\log (m)$ to the complexity.

\subsection{Nice tree decompositions}

A tree decomposition $(T, X)$ is called a nice tree decomposition if the following conditions are satisfied: Every node of the tree $T$ has at most two children; if a node $i$ has two children $j$ and $k$, then $X_{i}=X_{j}=X_{k}$; and if a node $i$ has one child $j$, then either $\left|X_{i}\right|=\left|X_{j}\right|+1$ and $X_{j} \subset X_{i}$ or $\left|X_{i}\right|=\left|X_{j}\right|-1$ and $X_{i} \subset X_{j}$. The following result is from (79):

Lemma 6.3.1. A tree decomposition $(T, X)$ of a graph $G$ can be transformed without increasing its width into a nice tree decomposition of $G$ in time polynomial in $|X|$ and the size of $G$. The size of the resulting nice tree decomposition is $O(w|X|)$, where $w$ is the width of the tree decomposition.

We point out that any tree decomposition of $G$ can be transformed into a tree decomposition of the same width having at most $n$ bags by simply removing all bags that are subset of another bag. Hence using Fact 6.3.1 we assume from here on that our algorithms run on nice tree decompositions with $\mathrm{O}(w n)$ bags, rooted in some arbitrary bag $X_{r}$. 


\subsection{DP for text graphs with bounded degree}

In this section, we consider problem Minimum $H$-Subgraph Edge Deletion in the setting where text graph $G$ has bounded degree and bounded treewidth and $\mathscr{H}$ consists of a single fixed, connected pattern $H$. We construct a dynamic programming algorithm for this setting that solves Minimum $H$-Subgraph EdGe Deletion in time that is exponential only in the maximum degree of $G$, in the width of a tree decomposition of $G$ and in some fixed parameters of $H$. A generalization of this result to the setting where $\mathscr{H}$ is a finite set of fixed, connected patterns will be introduced in Section 6.7 .

\subsection{1 constant parameters and notation}

By $w$, we denote the width of $(T, X)$. The maximum degree in $G$ will be denoted by $\Delta(G)$. The diameter of a graph $G=(V, E)$ is the maximum over all vertex pairs $\{u, v\} \subseteq V$ of the length of a shortest path between $u$ and $v$ in $G$. By $h$ and $d$ we denote respectively the size $|H|$ and the diameter of pattern $H$. Since $G$ has bounded degree and bounded treewidth and $H$ is a fixed connected pattern, the values $\Delta(G), w, h$ and $d$ are all constants in the dynamic program.

Given a bag $X$ in a nice tree decomposition $(T, X)$, we let $T_{X}$ be a subtree of $T$ that is rooted in $X$ and we let $G\left[T_{X}\right]$ be the subgraph of $G$ that is induced by all vertices from the bags in $T_{X}$. By $\mathscr{E}_{X}$ we denote the set of all edges of $G$ for which both end points are present in bag $X$ and by $E_{X}$, we denote a subset of $\mathscr{E}_{X}$, i.e., $E_{X} \in 2^{\mathscr{E}_{X}}$. For a bag $X$, by $V_{X}$ we denote the set of vertices of $G$ that are in bag $X$. For a subtree $T_{X}$, by $V_{T_{X}}$ we denote the set of vertices of $G$ that are present in some bag of $T_{X}$. Finally for a vertex set $S$, by $\mathscr{H}_{S}$ we denote the set of different $H$-subgraphs in $G$ that are incident to some vertex in $S$ and by $H_{S}$, we denote a subset of $\mathscr{H}_{S}$.

Lemma 6.4.1. Consider a graph $G$ and a tree decomposition $(T, X)$ of $G$ of width $w$. Let $Q=h !\left(\begin{array}{c}\Delta(G)^{d+1} \\ h\end{array}\right)$, then

- for vertex $v,\left|\mathscr{H}_{\{v\}}\right|$ is bounded from above by $Q$, and

- for bag $X$ in $(T, X)$, the value $\left|\mathscr{H}_{V_{X}}\right|$ is bounded from above by $(w+1) Q$.

Proof. Given that $v$ corresponds to some vertex of an $H$-subgraph in $G$ it is clear that all $h$ vertices in this $H$-subgraph have distance at most $d$ to $v$ in this $H$-subgraph and thus also in $G$. Since the maximum degree in $G$ is $\Delta(G)>1$, there are at most $\sum_{i=0}^{d} \Delta(G)^{i}=\frac{\Delta(G)^{d+1}-1}{\Delta(G)-1} \leq$ $\Delta(G)^{d+1}$ vertices in $G$ that have distance at most $d$ to $v$. Since $\left(\begin{array}{c}\Delta(G)^{d+1} \\ h\end{array}\right)$ different sets of $h$ vertices can be chosen among $\Delta(G)^{d+1}$ vertices and each such set can contain at most $h$ ! different $H$-subgraphs, the result follows. The second statement is a straightforward corollary of the first one, since $|X| \leq w+1$. 


\subsection{2 dynamic program and results}

First we note that by deleting a set of edges from $G$ that covers all $H$-subgraphs of $G, G$ becomes $H$-free. Given a subtree $T_{X}$ of $T$, we define:

- $F\left(E_{X}, T_{X}, H_{V_{X}}\right)$ is the minimum cardinality of a set $S$ of edges from $G\left[T_{X}\right]$ covering all $H$-subgraphs from $\left(\mathscr{H}_{V_{T_{X}}} \backslash \mathscr{C}_{V_{X}}\right) \cup H_{V_{X}}$ in $G$, given that $S \cap \mathscr{E}_{X}=E_{X}$

- $F\left(E_{X}, T_{X}, H_{V_{X}}\right)=\infty$ if $H$-subgraphs from $\left(\mathscr{H}_{V_{T_{X}}} \backslash \mathscr{H}_{V_{X}}\right) \cup H_{V_{X}}$ can $n o t$ be covered by $E_{X}$ plus some set of edges from $E\left(G\left[T_{X}\right]\right) \backslash \mathscr{E}_{X}$.

For each bag $X$ in $(T, X)$, we compute a table of such $F$-values for subtrees rooted in $X$, one value for each combination of a subset from $\mathscr{E}_{X}$ and a subset from $\mathscr{H}_{V_{X}}$. One such table thus contains $2^{\left|\mathscr{E}_{X}\right|+\left|\mathcal{H}_{V_{X}}\right|}$ values. To be more specific, we compute tables for the following three types of subtrees:

1. For each bag $X$ in $T$, except for root bag $X_{r}$, we compute the table for the subtree consisting of the parent $X^{+}$of $X, X$ and all descendants of $X$ in $T$. The root bag of this subtree is $X^{+}$. Such subtree will be denoted by $T_{X^{+}}$.

2. For each bag $X$ with two children we compute a table for the subtree $T_{X}$, consisting of bag $X$ and all descendants of $X$.

3. For each leaf bag $X$ we compute a table for the subtree $T_{X}$.

Note that for a leaf bag $X$, subtree $T_{X}$ equals bag $X$. Therefore the tables for the subtrees of type 3 are easy to compute.

Lemma 6.4.2. A value in the table of the subtree $T_{X}$ rooted in a leaf bag can be computed in constant time.

Proof. To compute one value $F\left(E_{X}, T_{X}, H_{V_{X}}\right)$ for the table of the subtree $T_{X}$ that is rooted in leaf bag $X$, one just needs to check whether edge set $E_{X}$ covers all $H$-subgraphs of $H_{V_{X}}$. If this is the case, then $F\left(E_{X}, T_{X}, H_{V_{X}}\right)=\left|E_{X}\right|$, else $F\left(E_{X}, T_{X}, H_{V_{X}}\right)=\infty$. Using the observation that $\left|E_{X}\right| \leq w^{2}$, Lemma 6.4.1 and the fact that both $w$ and $Q$ are constants, the result follows.

The following lemmas give recursive formulas that show how to compute the tables for subtrees that are rooted in a non-leaf bag of tree decomposition $(T, X)$. First we show how to update the table when we combine two subtrees that are rooted in the same bag and have only this bag in common. 
Lemma 6.4.3. Let $T_{X}^{\prime}$ and $T_{X}^{\prime \prime}$ be two subtrees rooted in $X$ that only share bag $X$. Let $T_{X}$ be the subtree that is obtained by combining $T_{X}^{\prime}$ and $T_{X}^{\prime \prime}$ and let $H_{V_{X}}, I_{V_{X}}, J_{V_{X}} \in 2^{\mathcal{B} V_{V_{X}} \text {. Then }}$

$$
F\left(E_{X}, T_{X}, H_{V_{X}}\right)=\min _{I_{V_{X}}, J_{V_{X}}: H_{V_{X}} \subseteq I_{V_{X}} \cup J_{V_{X}}} F\left(E_{X}, T_{X}^{\prime}, I_{V_{X}}\right)+F\left(E_{X}, T_{X}^{\prime \prime}, J_{V_{X}}\right)-\left|E_{X}\right| .
$$

The next two lemmas show how to update the values for a subtree when we extend it by the parent bag of its root.

Lemma 6.4.4. Let $T_{Y}$ be a subtree rooted in $Y$ and let $X=Y \cup\{v\}$ be the parent bag of $Y$. Furthermore let $T_{X}=T_{Y^{+}}$and let $E_{Y}=E_{X} \cap \mathscr{E}_{Y}$. Then:

$$
F\left(E_{X}, T_{X}, H_{V_{X}}\right)=\min _{H_{V_{Y}}: E_{X} \backslash E_{Y} \text { covers } H_{V_{X}} \backslash H_{V_{Y}} \text { in } G} F\left(E_{Y}, T_{Y}, H_{V_{Y}}\right)+\left|E_{X} \backslash E_{Y}\right|
$$

and $F\left(E_{X}, T_{X}, H_{V_{X}}\right)=\infty$ if there is no such $H_{V_{Y}}$.

Lemma 6.4.5. Let $T_{Y}$ be a subtree rooted in $Y$ and let $X=Y \backslash\{v\}$ be the parent bag of $Y$. Furthermore, let $T_{X}=T_{Y^{+}}$. Then:

$$
F\left(E_{X}, T_{X}, H_{V_{X}}\right)=\min _{E_{Y}, H_{V_{Y}}: H_{V_{X}} \cup\left(\mathscr{\mathcal { E }}_{\{v\}} \backslash \mathcal{H}_{V_{X}}\right) \subseteq H_{V_{Y}}, E_{Y} \cap \mathscr{E}_{X}=E_{X}} F\left(E_{Y}, T_{Y}, H_{V_{Y}}\right) .
$$

Obviously, when bag $Y$ and parent bag $X$ of $Y$ have the same vertex set, then the update of the table after an extension by the parent bag can be accomplished by simply copying the values. The following lemma gives an upper bound on the time to compute one $F$-value.

Lemma 6.4.6. The time needed to determine an F-value by one of the Lemmas 6.4.3, 6.4.4 or 6.4.5 is bounded from above by $O\left(2^{w^{2}+2(w+1) Q} w^{4} Q^{2}\right)$.

Proof. To determine a value using Lemma 6.4.3 takes $\mathrm{O}\left(4^{\left|\mathcal{H}_{V_{X}}\right|}\left|\mathscr{H}_{V_{X}}\right|^{2}\right)$ time. Using Lemma 6.4.1, this time is bounded from above by $\mathrm{O}\left(4^{(w+1) Q} w^{2} Q^{2}\right)$. To determine a value using Lemma 6.4.4, for all subsets $H_{V_{Y}}$ we have to check whether all elements from $H_{V_{X}} \backslash H_{V_{Y}}$ contain an edge from $E_{X} \backslash E_{Y}$. This takes less than $2^{\left|\mathcal{H}_{V_{Y}}\right|}\left|H_{V_{X}}\right|\left|E_{X}\right|$ time and by using Lemma 6.4.1 and the observation that $\left|E_{X}\right| \leq w^{2}$, this is bounded from above by $\mathrm{O}\left(2^{(w+1) Q} w^{3} Q\right)$. To determine a value using Lemma 6.4.5, for all combinations of $E_{Y}$ and $H_{V_{Y}}$ we have to check whether $H_{V_{X}} \subseteq H_{V_{Y}}$, whether $\mathscr{H}_{\{v\}} \backslash \mathscr{H}_{V_{X}} \subseteq H_{V_{Y}}$ and whether $E_{Y} \cap \mathscr{E}_{X}=E_{X}$. This takes less than $2^{\left|\mathscr{E}_{Y}\right|+\left|\mathcal{H}_{V_{Y}}\right|}\left(\left|H_{V_{X}}\right|+\left|\mathscr{H}_{V_{X}}\right|^{2}+\left|E_{X}\right|^{2}\right)$ time and as shown before this can be bounded by $\mathrm{O}\left(2^{w^{2}+(w+1) Q} w^{4} Q^{2}\right)$. Clearly, all these running times are smaller than $\mathrm{O}\left(2^{w^{2}+2(w+1) Q} w^{4} Q^{2}\right)$.

Using Lemmas 6.4.2, 6.4.3, 6.4.4 and 6.4.5, we can compute all necessary tables. For all bags in the tree, in post order, we construct the tables for the subtree(s) rooted in this bag. The proof of Lemma 6.4.2 shows how to construct the table for a subtree rooted in leaf bag $X$. If $X$ is not a leaf bag, suppose $X$ has 1 child $Y$, then we may assume that earlier 
already we computed the table for subtree $T_{Y}$. By using Lemma 6.4.4 or 6.4.5 we then can compute the table for subtree $T_{X}=T_{Y^{+}}$from the table of $T_{Y}$. If $X$ is not a leaf bag and it has 2 children $X$ and $Y$, then by definition of a nice tree decomposition $X=Y=Z$ and we may assume that we already computed the tables for subtrees $T_{Y}$ and $T_{Z}$. By simply copying the values we can construct the tables for subtrees $T_{Y^{+}}$and $T_{Z^{+}}$from the tables of $T_{Y}$ respectively $T_{Z}$. Then we use Lemma 6.4.3 to compute the table for subtree $T_{X}$ from the tables for subtrees $T_{Y^{+}}$and $T_{Z^{+}}$. The answer to Minimum $H$-Subgraph Edge Deletion can be found in the table of $T_{X_{r}}$. To be more precise, the solution is

$$
\min _{E_{X_{r}}} F\left(E_{X_{r}}, T_{X_{r}}, \mathscr{H}_{V_{X_{r}}}\right)
$$

Using the optimal set $E_{X_{r}}$, one can perform a backward search in the tree to determine a minimum set of edges that must be deleted from $G$ to make it $H$-free. To estimate the running time of the dynamic program, in the following lemma we determine an upper bound on the number of individual $F$-values that must be computed:

Lemma 6.4.7. In the dynamic program, at most $O\left(n 2^{w^{2}+(w+1) Q} w\right) F$-values need to be determined.

Proof. A bag in the rooted tree $T$ has at most 2 children. Thus for any bag $X$, we compute a table for at most 3 subtrees rooted in bag $X$, namely $T_{X}$ and $T_{Y^{+}}, T_{Z^{+}}$for possible children $Y, Z$ of $X$. We recall that the number of bags in $T$ is bounded by $\mathrm{O}(w n)$. Therefore the total number of tables to construct is bounded by $\mathrm{O}(w n)$. Since the width of $(T, X)$ is $w$, there are at most $w+1$ vertices in bag $X$ and therefore $\left|\mathscr{E}_{X}\right|$ is bounded from above by $\frac{w^{2}+w}{2} \leq w^{2}$ for $w \geq 1$. Furthermore, by Lemma 6.4.1, $\left|\mathscr{H}_{V_{X}}\right|$ is bounded from above by $(w+1) Q$. The latter two observations imply that the number of values in one table is bounded from above by $2^{w^{2}+(w+1) Q}$, from which the result follows.

The main result of this section is described in the following theorem:

Theorem 6.4.8. Given a graph $G$ of bounded maximum degree $\Delta(G)$, a fixed connected pattern $H$ and a nice tree decomposition $(T, X)$ of $G$ of bounded width $w$. The problem MINIMUM $H$-SUBGRAPH EDGE DELETION on $G$ with $\mathscr{H}=\{H\}$ can be solved in time $O\left(n 2^{2 w^{2}+3(w+1) Q} w^{5} Q^{2}\right)$, where $Q=h !\left(\begin{array}{c}\Delta(G)^{d+1} \\ h\end{array}\right)$.

Proof. By Lemma 6.4.7, we need to compute at most $\mathrm{O}\left(n 2^{w^{2}+(w+1) Q} w\right) F$-values in the dynamic program that solves the problem and by Lemma 6.4.6, it takes at most $\mathrm{O}\left(2^{w^{2}+2(w+1) Q} w^{4} Q^{2}\right)$ time to compute one such value. Combining these results, we conclude that the dynamic program runs in $\mathrm{O}\left(n 2^{2 w^{2}} w^{5}\left(\begin{array}{c}w \\ h-1\end{array}\right) h^{2}\right)$ time. 


\subsection{Dynamic program for clique patterns}

In this section, we consider Minimum $H$-Subgraph Edge Deletion for the special case where $H$ is a clique and $G$ has bounded treewidth. Compared to Section 6.5 we thus drop the constraint that $G$ should have bounded vertex degree. We exploit the fact that every tree decomposition contains a bag with all vertices from the clique (see Lemma 2.4.2) and we find a polynomial time algorithm that again acts on a nice tree decomposition of the text graph $G$. It is important to state here that in this section we consider $H$-subgraphs of $G$ that are induced by the vertex set of $G\left[T_{X}\right]$, not by the vertex set of $G$.

\subsection{1 dynamic program and results}

For subtree $T_{X}$ of $T$ rooted in bag $X$, we define:

- $F\left(E_{X}, T_{X}\right)$ is the minimum cardinality of a set $S$ of edges from $G\left[T_{X}\right]$ that covers all $H$-subgraphs of $G\left[T_{X}\right]$, given that $S \cap \mathscr{E}_{X}=E_{X}$.

- $F\left(E_{X}, T_{X}\right)=\infty$, if not all $H$-subgraphs of $G\left[T_{X}\right]$ can be covered by $E_{X}$ plus some set of edges from $E\left(G\left[T_{X}\right]\right) \backslash \mathscr{E}_{X}$.

We compute tables for the same subtrees as in the previous section. Note that one table for a subtree rooted in bag $X$ now consists of $2^{\left|\mathscr{E}_{X}\right|} F$-values. Using similar arguments as those used in the previous section, it is easy to prove the following:

Lemma 6.5.1. During a run of the dynamic program, at most $O\left(n 2^{w^{2}} w\right)$ individual $F$-values have to be determined.

As in the previous section, a value for the table of a subtree that is rooted in a leaf bag can be computed in constant time. The following lemmas give recursive formulas that show how to compute the tables for subtrees that are rooted in a non-leaf bag of $T$. The first lemma shows how we can combine subtrees that are rooted in the same bag and have only this bag in common.

Lemma 6.5.2. Let $T_{X}$ be obtained by taking the union of subtrees $T_{X}^{\prime}$ and $T_{X}^{\prime \prime}$ such that the root $X$ of $T_{X}^{\prime}$ and $T_{X}^{\prime \prime}$ is the only bag that belongs to both subtrees. Then

$$
F\left(E_{X}, T_{X}\right)=F\left(E_{X}, T_{X}^{\prime}\right)+F\left(E_{X}, T_{X}^{\prime \prime}\right)-\left|E_{X}\right|
$$

The next two lemmas show how to update the values for a subtree when we extend it by the parent bag of its root. 
Lemma 6.5.3. Let $T_{Y}$ be a subtree of $T$ rooted in bag $Y$, let $X=Y \cup\{v\}$ be the parent bag of $Y$ in T. Furthermore let $T_{X}=T_{Y^{+}}$and let $E_{Y}=E_{X} \cap \mathscr{E}_{Y}$. Then:

$$
F\left(E_{X}, T_{X}\right)=\left\{\begin{array}{cl}
F\left(E_{Y}, T_{Y}\right)+\left|E_{X} \backslash E_{Y}\right|, & \text { if } E_{X} \text { covers all } H \text {-subgraphs of } \\
& G\left[T_{X}\right] \text { that are incident to vertex } v . \\
\infty, & \text { otherwise. }
\end{array}\right.
$$

Indeed, since $X$ is the only bag containing $v$ in $T_{X}$, it also contains all neighbors of $v$ in $G\left[T_{X}\right]$. Therefore, all edges of an $H$-subgraph of $G\left[T_{X}\right]$ that are incident to $v$ are part of $\mathscr{E}_{X}$ and thus if such an $H$-subgraph is not covered by $E_{X}$ then it is not covered at all.

Lemma 6.5.4. Let $T_{Y}$ be a subtree of $T$ rooted in bag $Y$, let $X=Y \backslash\{v\}$ be the parent bag of $Y$ in $T$ and let $T_{X}=T_{Y^{+}}$. Then

$$
F\left(E_{X}, T_{X}\right)=\min _{E_{Y}: E_{Y} \cap \mathscr{E}_{X}=E_{X}} F\left(E_{Y}, T_{Y}\right) .
$$

Again, when bag $Y$ and parent bag $X$ of $Y$ have the same vertex set, then the update of the table after an extension by the parent bag can be accomplished by simply copying the values. In the previous section, it is explained how Lemmas 6.5.2, 6.5.3 and 6.5.4 can be used to determine the tables for all necessary subtrees. The minimum value in the table of subtree $T_{X_{r}}$ is the solution to Minimum $H$-SubGraph EdGe Deletion.

Lemma 6.5.5. The time needed to determine an F-value in the algorithm is bounded from above by $O\left(2^{w^{2}} w^{4}\left(\begin{array}{c}w \\ h-1\end{array}\right) h^{2}\right)$.

Proof. Determining the value for a subtree rooted in a leaf bag or by using Lemma 6.5.2 takes constant time. When using Lemma 6.5.3, we check whether $E_{X}$ covers all $H$-subgraphs of $G\left[T_{X}\right]$ that are incident to $v$. Vertex $v$ has at most $w$ neighbors in $G\left[T_{X}\right]$, so we have to check for each of the at most $\left(\begin{array}{c}w \\ h-1\end{array}\right)$ different combinations of $(h-1)$ such neighbors whether they form an $h$-clique with $v$ in $G\left[T_{X}\right]$ for which all $\frac{h^{2}-h}{2}$ edges are in $\mathscr{E}_{X} \backslash E_{X}$. Since $\left|\mathscr{E}_{X}\right|$ is bounded by $\mathrm{O}\left(w^{2}\right)$, this takes at most $\mathrm{O}\left(w^{2}\left(\begin{array}{c}w \\ h-1\end{array}\right) h^{2}\right)$ time. When using Lemma 6.5.4 to determine an $F$-value, for all $E_{Y}$ we have to check whether $E_{Y} \cap \mathscr{E}_{X}=E_{X}$, which can be done in time $\mathrm{O}\left(2^{w^{2}} w^{4}\right)$. Clearly, all these algorithmic time complexities are bounded from above by $\mathrm{O}\left(2^{w^{2}} w^{4}\left(\begin{array}{c}w \\ h-1\end{array}\right) h^{2}\right)$.

The main result of this section is described in the following theorem:

Theorem 6.5.6. Given an arbitrary text graph $G$, clique pattern $H$ of size $h$ and a nice tree decomposition $(T, X)$ of $G$ of bounded width $w$ with $N$ bags. Then Minimum $H$-SUBGRAPH EDGE DELETION on graph $G$ with $\mathscr{H}=\{H\}$ can be solved in $O\left(n 2^{2 w^{2}} w^{5}\left(\begin{array}{c}w \\ h-1\end{array}\right) h^{2}\right)$ time.

Proof. By Lemma 6.5.1, we need to compute at most $\mathrm{O}\left(n 2^{w^{2}} w\right) F$-values in the dynamic program that solves the problem and by Lemma 6.5 .5 , it takes at most $\mathrm{O}\left(2^{w^{2}} w^{4}\left(\begin{array}{c}w \\ h-1\end{array}\right) h^{2}\right)$ time to compute one such value. Combining these results, we conclude that the dynamic program runs in $\mathrm{O}\left(n 2^{2 w^{2}} w^{5}\left(\begin{array}{c}w \\ h-1\end{array}\right) h^{2}\right)$ time. 


\subsection{Baker's approximation scheme}

In this section, we consider the problem Minimum $H$-Subgraph Edge Deletion for planar text graphs and patterns. We combine the dynamic programs from Sections 6.4 and 6.4 with a technique invented by Brenda Baker to construct PTASs for the two cases where respectively $G$ has bounded vertex degree and where $H$ is a 3-clique or 4-clique.

\subsection{1 bounded outerplanarity index}

The following two lemmas form the analogues to respectively Theorem 6.4.8 and Theorem 6.5.6. The only difference is that they assume planar input graphs with bounded outerplanarity index instead of regular input graphs with bounded treewidth.

Lemma 6.6.1. Given a planar text graph $G$ of bounded maximum degree $\Delta(G)$ and bounded outerplanarity index $l$ and a fixed connected pattern $H$, an optimal solution to MINIMUM $H$ SUbGRAPH EDGE DELETION can be obtained in time $O\left(n 2^{18 l^{2}+9 l Q} l^{5} Q^{2}\right)$, where $Q=h !\left(\begin{array}{c}\Delta(G)^{d+1} \\ h\end{array}\right)$.

Proof. By Theorem 2.2.2, the outerplanarity index $l$ of $G$ can be determined in $\mathrm{O}\left(n^{2}\right)$ time. By Theorem 2.2.3, $t w(G) \leq 3 l-1$ and by Theorem 2.4.4, a tree decomposition of $G$ of width $w \leq 3 l-1$ can be obtained in linear time that can be turned into a nice tree decomposition in linear time. By Theorem 6.4.8 we can use this nice tree decomposition to solve MINIMUM $H$-SubGRAPH EdGe Deletion on $G$ in time $\mathrm{O}\left(n 2^{2 w^{2}+3(w+1) Q} w^{5} Q^{2}\right)$. Since $w \leq 3 l-1$, the result follows.

A similar result can be obtained for Minimum $H$-Subgraph Edge Deletion on planar graphs when $H$ is a 3-clique or 4-clique. Note that to cover all $K_{2}$-subgraphs of a graph by edges, one simply has to select as an Edge Cover the complete set of edges. Moreover, note that a planar graph $G$ does not have $K_{k}$ as a subgraph for $k \geq 5$. This is why we only consider $K_{3}$ and $K_{4}$ as subgraphs in this section that deals with planar graphs.

Lemma 6.6.2. Given a planar text graph $G$ of bounded outerplanarity index $l$ and pattern $H$ that is either $a K_{3}$ or $K_{4}$, an optimal solution to MINIMUM $H$-SUBGRAPH EDGE DELETION on $G$ can be obtained in time $O\left(n 2^{18 l^{2}} l^{5}\left(\begin{array}{c}3 l-1 \\ h-1\end{array}\right) h^{2}\right)$.

Proof. Similar as the proof of Lemma 6.6.1. By Theorem 6.5.6, Minimum $H$-SubGRAPH EDGe Deletion on $G$ can be solved in $\mathrm{O}\left(n 2^{2 w^{2}} w^{5}\left(\begin{array}{c}w \\ h-1\end{array}\right) h^{2}\right)$ time. Since $w \leq 3 l-1$, the result follows.

The problem Minimum $H$-Subgraph Edge Deletion on planar graphs of bounded degree with fixed, connected pattern is thus fixed parameter tractable, since it is tractable 
when parameterized by outerplanarity index $l$ of $G$. The same holds for Minimum $H$ SUBGRAPH EDGE DELETION on planar graphs with clique pattern. We will use these properties to construct a Baker's approximation scheme in the next section that can be applied to both problems.

\subsection{2 approximation schemes}

Given a planar embedding of a planar graph $G=(V, E)$, we say that a vertex $v$ is of level 1 if it is on the exterior face of the embedding. Let $V_{i}$ be the set of all vertices of level $i$ or lower than $i$, then vertex $w$ is in level $i+1$ if it is on the exterior face of the embedding induced by $G\left[V \backslash V_{i}\right]$. We say that edge $e=(v, w) \in E$ is in level $i$ of the embedding if both vertices $v$ and $w$ are in level $i$. We assume in this section that a planar embedding is represented by an appropriate data structure such that levels of vertices can be computed in linear time.

In the following two theorems and their proof, we denote by $E_{o p t}$ a minimum set of edges from $G$ covering all $H$-subgraphs of $G$ and by $O P T$ we denote the size of $E_{\text {opt }}$. First we present an approximation scheme for Minimum $H$-Subgraph EdGe Deletion on a planar graph $G$ with bounded degree and arbitrary fixed, connected pattern $H$. A generalization of the results to finite sets of fixed, connected patterns will be introduced in Section 6.7 .

Theorem 6.6.3. For a planar text graph $G$ of bounded degree, a fixed connected pattern $H$ and any $s>0$, there is a $O\left(n^{2} 2^{18 l^{2}+9 l Q} l^{5} Q^{2}\right)$-time algorithm for MINIMUM $H$-SUBGRAPH EDGE DELETION that finds a solution of size at most $\left(\frac{s+1}{s}\right) O P T$, where $l$ is a constant depending on $s$ and the outerplanarity index of $H$.

Proof. First, we use Theorem 2.2.2 to determine $G$ 's outerplanarity index $k$ and a $k$-outerplanar embedding of $G$ in $\mathrm{O}\left(n^{2}\right)$ time. I.e, each vertex of $G$ belongs to one of the $k$ levels. Similarly, we determine $H$ 's outerplanarity index $k^{\prime}$ in $\mathrm{O}\left(h^{2}\right)$ time. Since $H$ is a fixed subgraph, $k^{\prime}$ is a constant. If $k$ was a constant, the problem could be solved in polynomial time by complete enumeration. Therefore it is reasonable to assume that $k>(s+2) k^{\prime}$. Now for some fixed $2 k^{\prime} \leq l \leq k$ and for each $i \in I=\left\{m k^{\prime}+1 \mid 0 \leq m \leq\left\lfloor\frac{l}{k^{\prime}}-2\right\rfloor\right\}$ we construct a set $G^{i}$ of induced subgraphs of $G$, consisting of the $l$-outerplanar subgraphs of $G$ :

- induced by levels 1 to $i+k^{\prime}-1$.

- induced by levels $j\left(l-k^{\prime}\right)+i$ to $j\left(l-k^{\prime}\right)+i+l-1,0 \leq j \leq\left\lfloor\frac{k-i-l+1}{l-k^{\prime}}\right\rfloor$.

- induced by levels $\left\lfloor\frac{k-i-l+1}{l-k^{\prime}}\right\rfloor\left(l-k^{\prime}\right)+i+l-k^{\prime}$ to $k$.

See Figure 6.1 for an illustration. Note that $i+k^{\prime}-1 \leq\left\lfloor\frac{l}{k^{\prime}}-2\right\rfloor k^{\prime}+1+k^{\prime}-1 \leq l-k^{\prime}<l$ 


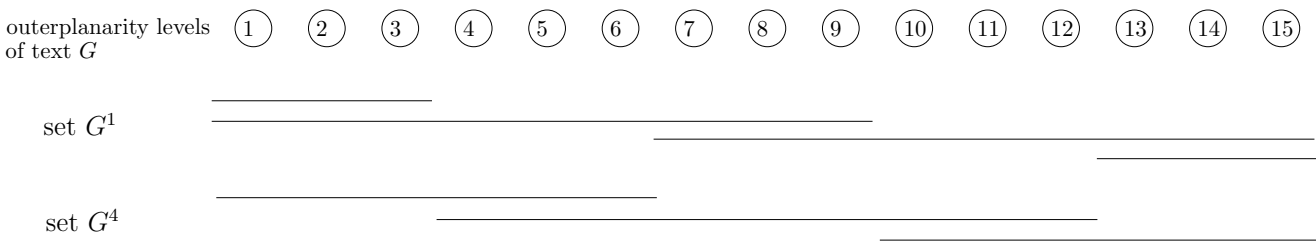

Figure 6.1: Suppose text $G$ has 15 outerplanarity levels and pattern $H$ is 3 -outerplanar. For $l=9$, we construct two sets $\left(G^{1}\right.$ and $\left.G^{4}\right)$ of 9-outerplanar subgraphs of $G$. For example, the first graph in $G^{1}$ is induced by all vertices in $G$ 's first three levels.

and also that $k-\left(\left\lfloor\frac{k-i-l+1}{l-k^{\prime}}\right\rfloor\left(l-k^{\prime}\right)+i+l-k^{\prime}\right)+1 \leq k-\left(k-i-2 l+1+k^{\prime}+i+l-k^{\prime}\right)+1=l$, so both the subgraph induced by the first bullet and the one induced by the third bullet are $l$-outerplanar. Clearly, also the subgraphs under the second bullet are $l$-outerplanar. Since $|I|=\left\lfloor\frac{l}{k^{\prime}}-1\right\rfloor$ and for each $i$ we construct $\left\lfloor\frac{k-i-l+1}{l-k^{\prime}}+3\right\rfloor$ subgraphs, in total we construct less than $\left(\frac{l-k^{\prime}}{k^{\prime}}\right)\left(\frac{k+2 l+1}{l-k^{\prime}}\right)=\frac{k+2 l+1}{k^{\prime}} \leq 3 k \leq 3 n=\mathrm{O}(n)$ induced subgraphs of $G$ for fixed value of $l$.

Observation 6.6.4. For every $i \in I$, the vertices in the following set of levels are the only vertices that are part of more than one graph from $G^{i}$ :

- levels $j\left(l-k^{\prime}\right)+i, \ldots, j\left(l-k^{\prime}\right)+i+k^{\prime}-1,0 \leq j \leq\left\lfloor\frac{k-i-l+1}{l-k^{\prime}}\right\rfloor$ and

- levels $\left\lfloor\frac{k-i-l+1}{l-k^{\prime}}\right\rfloor\left(l-k^{\prime}\right)+i+l-k^{\prime}$ to $\left\lfloor\frac{k-i-l+1}{l-k^{\prime}}\right\rfloor\left(l-k^{\prime}\right)+i+l-1$.

Observation 6.6.5. For at least one $i \in I$ the set of levels from Observation 6.6.4 contains at most $\frac{k^{\prime}}{l-2 k^{\prime}} O P T$ edges from $E_{\text {opt }}$.

Proof. For any two different values of $i$ from $I$, the two sets of levels from Observation 6.6.4 are disjoint. Thus if for all $i \in I$, the sets of levels would contain strictly more than $\frac{k^{\prime}}{l-2 k^{\prime}} O P T$ edges from $E_{o p t}$, then all these levels would contain strictly more than

$$
\sum_{m=0}^{\left\lfloor\frac{l}{k^{\prime}}-2\right\rfloor} \frac{k^{\prime}}{l-2 k^{\prime}} O P T=\left\lfloor\frac{l}{k^{\prime}}-1\right\rfloor \frac{k^{\prime}}{l-2 k^{\prime}} O P T \geq\left(\frac{l-k^{\prime}}{k^{\prime}}-1\right) \frac{k^{\prime}}{l-2 k^{\prime}} O P T=O P T
$$

edges from $E_{o p t}$, a contradiction.

Now for each $i \in I$, we use Lemma 6.6.1 to compute optimal solutions to MINIMUM $H$ Subgraph Edge Deletion for all $l$-outerplanar subgraphs. Since for all values of $i$ together, the number of $l$-outerplanar subgraphs is bounded by $\mathrm{O}(n)$, this can be done in time $\mathrm{O}\left(n^{2} 2^{18 l^{2}+9 l Q} l^{5} Q^{2}\right)$. We observe that for each $i$, any $H$-subgraph of $G$ is present in at least one of the subgraphs of $G$ induced by this $i$. Therefore, for each $i$, the union of the optimal solutions for its induced subgraphs is a set of edges that covers all $H$-subgraphs in $G$. The algorithm picks the best of these unions as an approximation to the optimal 
solution. To see that this approximation is at most $\left(\frac{s+1}{s}\right) O P T$, consider again an optimal solution $E_{\text {opt }}$ for $G$. We pick the value $i$ that by Observations 6.6.4 and 6.6.5 has not more than $\frac{k^{\prime}}{l-2 k^{\prime}} O P T$ edges from $E_{\text {opt }}$ in intersecting levels of graphs from $G^{i}$. For each element $S \in G^{i}$ we let $E_{S}$ be the set of edges in $E_{o p t}$ in subgraph $S$. Furthermore, we let $E_{S}^{\prime}$ be the edges in an optimal solution of Minimum $H$-Subgraph Edge Deletion for $S$. For this choice of $i$, we thus have a solution of Minimum $H$-SubGRAPH EDGE DELETION for $G$ of size no larger than the sum of the $\left|E_{S}^{\prime}\right|$ 's. Clearly for each $S$ it holds that $\left|E_{S}^{\prime}\right| \leq\left|E_{S}\right|$. Moreover, since at most $\frac{k^{\prime}}{l-2 k^{\prime}} O P T$ edges are counted twice while summing the $\left|E_{S}\right|$ 's, we conclude that $\sum_{S \in G^{i}}\left|E_{S}^{\prime}\right| \leq \sum_{S \in G^{i}}\left|E_{S}\right| \leq \frac{k^{\prime}}{l-2 k^{\prime}} O P T+O P T=\frac{l-k^{\prime}}{l-2 k^{\prime}} O P T$. Thus in total time $\mathrm{O}\left(n^{2}\right)+\mathrm{O}\left(h^{2}\right)+\mathrm{O}\left(n^{2} 2^{18 l^{2}+9 l Q} l^{5} Q^{2}\right)$ we constructed a solution of size at most $\frac{l-k^{\prime}}{l-2 k^{\prime}} O P T$. By choosing $l=(s+2) k^{\prime}$, we obtain the $\left(\frac{s+1}{s}\right)$-approximation to $O P T$. Since $s$ is strictly positive and we assume that $k>(s+2) k^{\prime}$, we ensure that $2 k^{\prime} \leq l \leq k$.

Next, we present an approximation scheme for Minimum $H$-SubGRAPH EdGe DeleTION on planar graph $G$ and pattern $H$ that is either a $K_{3}$ or a $K_{4}$.

Theorem 6.6.6. For planar text graph $G$, pattern $H$ that is either a $K_{3}$ or $K_{4}$ and any $s>0$, there is a $O\left(n^{2} 2^{18 l^{2}} l^{5}\left(\begin{array}{c}3 l-1 \\ h-1\end{array}\right) h^{2}\right)$-time algorithm for MINIMUM $H$-SUBGRAPH EDGE DELETION on $G$ that finds a solution of size at most $\left(\frac{s+1}{s}\right) O P T$, where $l$ is a constant depending on $s$ and the outerplanarity index of $H$.

Proof. Same as proof of Theorem 6.6.3, with the only difference that Lemma 6.6.2 is used instead of Lemma 6.6.1.

\subsection{Generalization to set $\mathscr{H}$ of patterns}

In this final section of the chapter we generalize the results from the previous sections. We show how the algorithms can be adopted to deal with a finite set $\mathscr{H}=\left\{H_{1}, \ldots, H_{t}\right\}, t>1$ of fixed, connected patterns instead of with a single fixed, connected pattern $H$. Consider such a set of patterns $\mathscr{H}=\left\{H_{1}, \ldots, H_{t}\right\}, t>1$. First we note that if $H_{i} \in \mathscr{H}$ is a subgraph of $H_{j} \in \mathscr{H}, j \neq i$, then $H_{j}$ is redundant. Indeed, a set of edges that covers all occurrences of $H_{i}$ as a subgraph of $G$ will also cover all occurrences of $H_{j}$ as a subgraph of $G$. Therefore any graph $G$ that is $H_{i}$-free is also $H_{j}$-free. For the set of cliques this means that only the smallest clique is significant and hence there is no need to generalize the result from Section 6.5. We thus generalize the results from Section 6.4. To that end, we consider text graph $G$ of bounded maximum degree and a finite set $\mathscr{H}$ of fixed connected patterns such that for each $1 \leq i \neq j \leq t, H_{i}$ is not isomorphic to a subgraph of $H_{j}$. By $h_{i}, d_{i}$ and $k_{i}$ we denote respectively the size, the diameter and the outerplanarity index of pattern $H_{i}$. The following theorem then generalizes Theorem 6.4.8. 
Theorem 6.7.1. Given a text graph $G$ of bounded maximum degree $\Delta(G)$, a finite set of fixed, connected patterns $\mathscr{H}$ and a nice tree decomposition $(T, X)$ of $G$ of bounded width $w$, the problem Minimum $H$-SubGRAPH EdGe Deletion on $G$ can be solved in time $O\left(n 2^{2 w^{2}+3(w+1) Q} w^{5} Q^{2}\right)$, where $Q=\sum_{i=1}^{t} h_{i} !\left(\begin{array}{c}\Delta(G)^{d_{i}+1} \\ h_{i}\end{array}\right)$.

Proof. We repeat the proof of Theorem 6.4.8, taking $Q=\sum_{i=1}^{t} h_{i} !\left(\begin{array}{c}\Delta(G)^{d_{i}+1} \\ h_{i}\end{array}\right)$ as a new upper bound on the number of considered subgraphs of $G$.

Similarly, we generalize Theorem 6.6.3.

Theorem 6.7.2. For a planar text graph $G$ of bounded degree, finite set of fixed, connected patterns $\mathscr{H}$ and any $s>0$, there is a $O\left(n^{2} 2^{18 l^{2}+9 l Q} l^{5} Q^{2}\right)$-time algorithm for MINIMum $H$ SUbGRAPH EDGE DELETION that finds a solution of size at most $\left(\frac{s+1}{s}\right) O P T$, where $Q=$ $\sum_{i=1}^{t} h_{i} !\left(\begin{array}{c}\Delta(G)^{d_{i}+1} \\ h_{i}\end{array}\right)$ and $l$ is a constant depending on $s$ and the largest outerplanarity index over all elements from $\mathscr{H}$.

Proof. The proof is similar to the proof of Theorem 6.6.3, with the difference that $k^{\prime}=$ $\max _{1 \leq i \leq t} k_{i}$ to guarantee that each occurrence of a pattern from $\mathscr{H}$ as a subgraph of $G$ is present in at least one subgraph from $G^{i}, i \in I$.

\subsection{Conclusions}

We analyzed the problem of excluding a set $\mathscr{H}$ of patterns as subgraphs of a graph $G$ by deleting a (minimum) number of edges from $G$. By a general result from Courcelle and through a formulation of the problem in Monadic Second Order Logic, we implicitly showed that the decision version of the problem is solvable in linear time on graphs that have bounded treewidth. Subsequently, we presented a constructive algorithm solving the optimization version of the problem in linear time by using dynamic programming on a tree decomposition of the input graph. For this, however, we needed the additional assumption that the input graph has bounded maximum vertex degree, except for the case where $\mathscr{H}$ contains only cliques. Using Baker's layerwise decomposition approach, we created a polynomial time approximation scheme for the problem on planar graphs, using the same additional assumption. It should be noted that the constants in the time complexities of both the linear time algorithm as the PTAS are immense, severely limiting their practical usage. The question remains open whether a combinatorial linear time algorithm and a PTAS can be found that do not require the condition concerning maximum vertex degree in the input graph. 


\section{Bibliography}

[1] Scott Aarsonson: Complexity Zoo. April 2010,

http://qwiki.stanford.edu/wiki/Complexity_Zoo

[2] Jochen Alber, Hans L. Bodlaender, Henning Fernau, Ton Kloks, Rolf Niedermeier: Fixed Parameter Algorithms for DOMINATING SET and Related Problems on Planar Graphs. Algorithmica 33(4): 461-493 (2002)

[3] Jochen Alber, Rolf Niedermeier: Improved Tree Decomposition Based Algorithms for Domination-like Problems. LATIN 2002: 613-628

[4] Jochen Alber, Frederic Dorn, Rolf Niedermeier: Experimental evaluation of a tree decomposition-based algorithm for vertex cover on planar graphs. Discrete Applied Mathematics 145(2): 219-231 (2005)

[5] Eyal Amir: Efficient Approximation for Triangulation of Minimum Treewidth. UAI 2001: 7-15

[6] Stefan Arnborg: Efficient Algorithms for Combinatorial Problems with Bounded Decomposability - A Survey. BIT 25(1): 2-23 (1985)

[7] Stefan Arnborg, Derek G. Corneil, Andrzej Proskurowski: Complexity of finding embeddings in a k-tree. SIAM Journal on Algebraic and Discrete Methods 8(2): 277-284 (1987)

[8] Stefan Arnborg, Andrzej Proskurowski: Linear time algorithms for NP-hard problems restricted to partial k-trees. Discrete Applied Mathematics 23(1): 11-24 (1989)

[9] Stefan Arnborg, Jens Lagergren, Detlef Seese: Easy Problems for Tree-Decomposable Graphs. J. Algorithms 12(2): 308-340 (1991)

[10] Emgad H. Bachoore, Hans L. Bodlaender: A Branch and Bound Algorithm for Exact, Upper, and Lower Bounds on Treewidth. AAIM 2006: 255-266

[11] Brenda S. Baker: Approximation Algorithms for NP-Complete Problems on Planar Graphs. J. ACM 41(1): 153-180 (1994) 
[12] Ann Becker, Dan Geiger: A sufficiently fast algorithm for finding close to optimal clique trees. Artif. Intell. 125(1-2): 3-17 (2001)

[13] Marshall W. Bern, Eugene L. Lawler, A.L. Wong: Linear-Time Computation of Optimal Subgraphs of Cecomposable Graphs. J. Algorithms 8(2): 216-235 (1987)

[14] Zhengbing Bian, Qian-Ping Gu: Computing Branch Decomposition of Large Planar Graphs. WEA 2008: 87-100

[15] Daniel Bienstock, Clyde L. Monma: On the Complexity of Embedding Planar Graphs To Minimize Certain Distance Measures. Algorithmica 5(1): 93-109 (1990)

[16] Norman L. Biggs, E. K. Lloyd, Robin J. Wilson: Graph Theory: 1736-1936. Oxford: Clarendon Press (1976)

[17] Jean R.S. Blair, Pinar Heggernes, Jan Arne Telle: A practical algorithm for making filled graphs minimal. Theor. Comput. Sci. 250(1-2): 125-141 (2001)

[18] Hans L. Bodlaender: Dynamic Programming on Graphs with Bounded Treewidth. ICALP 1988: 105-118

[19] Hans L. Bodlaender, Rolf H. Möhring: The Pathwidth and Treewidth of Cographs. SIAM J. Discrete Math. 6(2): 181-188 (1993)

[20] Hans L. Bodlaender: Improved Self-reduction Algorithms for Graphs with Bounded Treewidth. Discrete Applied Mathematics 54(2-3): 101-115 (1994)

[21] Hans L. Bodlaender, John R. Gilbert, Hjálmtyr Hafsteinsson, Ton Kloks: Approximating Treewidth, Pathwidth, Frontsize, and Shortest Elimination Tree. J. Algorithms 18(2): 238-255 (1995)

[22] Hans L. Bodlaender, Ton Kloks, Dieter Kratsch: Treewidth and Pathwidth of Permutation Graphs. SIAM J.Discrete Math. 8(4): 606-616 (1995)

[23] Hans L. Bodlaender: A Linear-Time Algorithm for Finding Tree-Decompositions of Small Treewidth. SIAM J. Comput. 25(6): 1305-1317 (1996)

[24] Hans L. Bodlaender, Ton Kloks: Efficient and Constructive Algorithms for the Pathwidth and Treewidth of Graphs. J. Algorithms 21(2): 358-402 (1996)

[25] Hans L. Bodlaender, Dimitrios M. Thilikos: Treewidth for Graphs with Small Chordality. Discrete Applied Mathematics 79(1-3): 45-61 (1997)

[26] Hans L. Bodlaender: Treewidth: Algorithmic Techniques and Results. MFCS 1997: 1936

[27] Hans L. Bodlaender: A Partial k-Arboretum of Graphs with Bounded Treewidth. Theor. Comput. Sci. 209(1-2): 1-45 (1998) 
[28] Hans L. Bodlaender, Ton Kloks, Dieter Kratsch, Haiko Müller: Treewidth and Minimum Fill-in on d-Trapezoid Graphs. J. Graph Algorithms Appl. 2(2): 1-23 (1998)

[29] Hans L. Bodlaender, Udi Rotics: Computing the Treewidth and the Minimum Fill-In with the Modular Decomposition. Algorithmica 36(4): 375-408 (2003)

[30] Hans L. Bodlaender: Discovering Treewidth. SOFSEM 2005: 1-16

[31] Hans L. Bodlaender, Arie M. C. A. Koster, Frank van den Eijkhof: Preprocessing Rules for Triangulation of Probabilistic Networks. Computational Intelligence 21(3): 286305 (2005)

[32] Hans L. Bodlaender, Fedor V. Fomin, Arie M. C. A. Koster, Dieter Kratsch, Dimitrios M. Thilikos: On Exact Algorithms for Treewidth. ESA 2006: 672-683

[33] Hans L. Bodlaender, Thomas Wolle, Arie M. C. A. Koster: Contraction and Treewidth Lower Bounds. J. Graph Algorithms Appl. 10(1): 5-49 (2006)

[34] Hans L. Bodlaender, Arie M.C.A. Koster: Combinatorial Optimization on Graphs of Bounded Treewidth. Comput. J. 51(3): 255-269 (2008)

[35] Hans L. Bodlaender, Alexander Grigoriev, Arie M.C.A. Koster: Treewidth Lower Bounds with Brambles. Algorithmica 51(1): 81-98 (2008)

[36] Hans L. Bodlaender, Arie M. C. A. Koster: Treewidth computations I. Upper bounds. Inf. Comput. 208(3): 259-275 (2010)

[37] Tom Bohman: The triangle-free process. Advances in Mathematics 221(5): 1653-1677 (2009)

[38] Béla Bollobás: Extremal Graph Theory. London Mathematical Society Monographs, Vol. 11, Academic Press, London (1978)

[39] J.A. Bondy, U.S.R. Murty: Graph Theory. Springer Publishing Company, Incorporated (2008)

[40] Oleg V. Borodin, Alexei N. Glebov, André Raspaud, Mohammad R. Salavatipour: Planar Graphs without Cycles of Length from 4 to 7 are 3-Colorable. J. Comb. Theory, Ser. B 93(2): 303-311 (2005)

[41] Vincent Bouchitté, Ioan Todinca: Treewidth and Minimum Fill-in: Grouping the Minimal Separators. SIAM J. Comput. 31(1): 212-232 (2001)

[42] Vincent Bouchitté, Ioan Todinca: Listing all potential maximal cliques of a graph. Theor. Comput. Sci. 276(1-2): 17-32 (2002)

[43] Vincent Bouchitté, Ioan Todinca: Approximating the treewidth of AT-free graphs. Discrete Applied Mathematics 131(1): 11-37 (2003) 
[44] Vincent Bouchitté, Dieter Kratsch, Haiko Müller, Ioan Todinca: On treewidth approximations. Discrete Applied Mathematics 136(2-3): 183-196 (2004)

[45] Andreas Brandstädt, Van Bang Le, Jeremy P. Spinrad: Graph classes: a survey. Society for Industrial and Applied Mathematics, Philadelphia, PA (1999)

[46] Hajo Broersma, Elias Dahlhaus, Ton Kloks: A Linear Time Algorithm for Minimum Fill-in and Treewidth for Distance Hereditary Graphs. Discrete Applied Mathematics 99(1-3): 367-400 (2000)

[47] Hajo Broersma, Ton Kloks, Dieter Kratsch, Haiko Müller: A Generalization of AT-Free Graphs and a Generic Algorithm for Solving Triangulation Problems. Algorithmica 32(4): 594-610 (2002)

[48] Daniel Brügmann, Christian Komusiewicz, Hannes Moser: On Generating TriangleFree Graphs. Electronic Notes in Discrete Mathematics 32: 51-58 (2009)

[49] François Clautiaux, Jacques Carlier, Aziz Moukrim, Stéphane Nègre: New Lower and Upper Bounds for Graph Treewidth. WEA 2003: 70-80. Lecture Notes in Computer Science 2647, Springer-Verlag (2003)

[50] François Clautiaux, Aziz Moukrim, Stéphane Nègre, Jacques Carlier: Heuristics and metaheuristic methods for computing graph treewidth. RAIRO - Operations Research 38(1): 13-26 (2004)

[51] Bruno Courcelle: The Monadic Second-Order Logic of Graphs. I. Recognizable Sets of Finite Graphs. Inf. and Comput. 85(1): 12-75 (1990)

[52] Bruno Courcelle: The monadic second-order logic of graphs III: tree-decompositions, minor and complexity issues. ITA 26: 257-286 (1992)

[53] Elias Dahlhaus: Minimum Fill-in and Treewidth for Graphs Modularly Decomposable into Chordal Graphs. WG 1998: 351-358

[54] Erik D. Demaine, MohammadTaghi Hajiaghayi: Approximation Schemes for Planar Graph Problems. Encyclopedia of Algorithms 2008

[55] Erik D. Demaine, MohammadTaghi Hajiaghayi: Bidimensionality. Encyclopedia of Algorithms 2008

[56] Reinhard Diestel, Tommy R. Jensen, Konstantin Yu. Gorbunov, Carsten Thomassen: Highly Connected Sets and the Excluded Grid Theorem. J. Comb. Theory, Ser. B 75(1): 61-73 (1999)

[57] Reinhard Diestel: Graph Theory. Fourh Edition, Springer-Verlag, Heidelberg (2010)

[58] Jean E. Dunbar, Marietjie Frick: The Path Partition Conjecture is true for claw-free graphs. Discrete Mathematics 307(11-12): 1285-1290 (2007) 
[59] Paul Erdös, Daniel J. Kleitman, Bruce Rothschild: Asymptotic Enumeration of $K_{n}$-free Graphs. Atti Dei Convegni Lincei 17, Colloquio Internazionale sulle Teorie Combinatorie 20: 19-27, American Mathematical Society, Rome (1976)

[60] Fedor V. Fomin, Dieter Kratsch, Ioan Todinca: Exact (Exponential) Algorithms for Treewidth and Minimum Fill-In. ICALP 2004: 568-580

[61] Fedor V. Fomin, Dieter Kratsch, Ioan Todinca, Yngve Villanger: Exact Algorithms for Treewidth and Minimum Fill-In. SIAM J. Comput. 38(3): 1058-1079 (2008)

[62] Fedor V. Fomin, Yngve Villanger: Treewidth Computation and Extremal Combinatorics. ICALP(1)2008: 210-221

[63] Michael R. Garey, David S. Johnson: Computers and Intractability; A Guide to the Theory of NP-Completeness. W.H. Freeman \& Co., New York, NY (1990)

[64] Vibhav Gogate, Rina Dechter: A complete Anytime Algorithm for Treewidth. UAI 2004: 201-208

[65] Martin Charles Golumbic, Haim Kaplan, Ron Shamir: Graph Sandwich Problems. J. Algorithms 19(3): 449-473 (1995)

[66] Graph Classes,

http://wwwteo.informatik.uni-rostock.de/isgci/classes.cgi

[67] Alexander Grigoriev: Treewidth and large grid minors in planar graphs. unpublished manuscript (2007)

[68] Jonathan L. Gross, Jay Yellen: Graph Theory and Its Applications. Second Edition (Discrete Mathematics and Its Applications), Chapman \& Hall/CRC (2005)

[69] Qian-Ping Gu, Hisao Tamaki: Optimal Branch-Decomposition of Planar Graphs in $O\left(n^{3}\right)$ Time. ACM Transactions on Algorithms 4(3): (2008)

[70] Qian-Ping Gu, Hisao Tamaki: Improved bounds on the planar branchwidth with respect to the largest grid minor size. Technical Report 2009-17: School of Computing Science, Simon Fraser University, Burnaby, BC, Canada (2009)

[71] Michel Habib, Rolf H. Möhring: Treewidth of cocomparability graphs and a new ordertheoretic parameter. ORDER 11(1): 47-60 (1994)

[72] Illya V. Hicks: Planar Branch Decompositions I: The Ratcatcher. INFORMS Journal on Computing (INFORMS) 17(4): 402-412 (2005)

[73] Illya V. Hicks: Planar Branch Decompositions II: The Cycle Method. INFORMS Journal on Computing (INFORMS) 17(4): 413-421 (2005)

[74] Illya V. Hicks: Graphs, Branchwidth, and Tangles! Oh my! Networks 45(2): 55-60 (2005) 
[75] Frank Kammer: Determining the Smallest $k$ Such That G Is k-Outerplanar. ESA 2007: $359-370$

[76] Pierluigi Crescenzi, Viggo Kann:

A compendium of NP optimization problems. July 2005, http://www. nada.kth.se/ viggo/wwwcompendium/wwwcompendium.html

[77] Ton Kloks, Dieter Kratsch: Treewidth of Chordal Bipartite Graphs. J. Algorithms 19(2): 266-281 (1995)

[78] Ton Kloks: Treewidth of Circle Graphs. Int. J. Found. Comput. Sci. 7(2): 111-120 (1996)

[79] Ton Kloks: Treewidth: Computations and Approximations. Lecture Notes in Computer Science 842 Springer-Verlag, Heidelberg, (1994)

[80] Ton Kloks, Dieter Kratsch, Jeremy Spinrad: On Treewidth and Minimum Fill-In of Asteroidal Triple-Free Graphs. Theor. Comput. Sci. 175(2): 309-335 (1997)

[81] Arie M.C.A. Koster: Frequency assignment - models and algorithms. PhD thesis, University of Maastricht, Maastricht, The Netherlands, (1999)

[82] Arie M.C.A. Koster, Stan P.M. van Hoesel, Antoon W.J. Kolen: Solving Frequency Assignment Problems via Tree-Decompositions. Electronic Notes in Discrete Mathematics 3: 102-105 (1999)

[83] Arie M.C.A. Koster, Hans L. Bodlaender, Stan P.M. van Hoesel: Treewidth: Computational Experiments. Electronic Notes in Discrete Mathematics 8: 54-57 (2001)

[84] Arie M.C.A. Koster, Stan P.M. van Hoesel, Antoon W.J. Kolen: Solving partial constraint satisfaction problems with tree decompositions. Networks 40(3): 170-180 (2002)

[85] K. Kuratowski: Sur le problème des courbes gauches en topologie. Fund. Math. 15: 271-283 (1930)

[86] Jens Lagergren, Stefan Arnborg: Finding Minimal Forbidden Minors Using a Finite Congruence. ICALP 1991: 532-543

[87] Jens Lagergren: Efficient Parallel Algorithms for Graphs of Bounded Tree-Width. J. Algorithms 20(1): 20-44 (1996)

[88] Steffen L. Lauritzen, David J. Spiegelhalter: Local computations with probabilities on graphical structures and their application to expert systems. Journal of the Royal Statistical Society, series B 50: 253-258 (1988)

[89] C.G. Lekkerkerker, J.C. Boland: Representation of finite graphs by a set of intervals on the real line. Fund. Math. 51: 45-64 (1962) 
[90] Jan van Leeuwen: Graph Algorithms. Handbook of Theoretical Computer Science, Volume A: Algorithms and Complexity, 522-631 (1990)

[91] Jean-Francois Manouvrier, Corinne Lucet: Resolving the Network Reliability Problem with a Tree Decomposition of the Graph. OPODIS 1997: 193-204

[92] George J. Minty: On Maximal Independent Sets of Vertices in Claw-free Graphs. J. Comb. Theory, Ser. B 28(3): 284-304 (1980)

[93] Bruce A. Reed: Finding Approximate Separators and Computing Tree Width Quickly. STOC 1992: 221-228

[94] Neil Robertson, Paul D. Seymour: Graph minors. III. Planar tree-width. J. Comb. Theory, Ser. B 36(1): 49-64 (1984)

[95] Neil Robertson, Paul D. Seymour: Graph minors. II. Algorithmic Aspects of Tree-Width. J. Algorithms 7(3): 309-322 (1986)

[96] Neil Robertson, Paul D. Seymour: Graph minors. X. Obstructions to tree-decomposition. J. Comb. Theory, Ser. B 52(2): 153-190 (1991)

[97] Donald J. Rose: A Graph-Theoretic Study of the Numerical Solution of Sparse Positive Definite Systems of Linear Equations. Graph Theory and Computing, 183-217 (1972)

[98] Donald J. Rose, Robert E. Tarjan, George S. Lueker: Algorithmic Aspects of Vertex Eliminination on Graphs. SIAM J. Comput. 5(2): 266-283 (1976)

[99] Najiba Sbihi: Algorithme de Recherche d'un Stable de Cardinalité Maximum dans un Graphe sans Étoile. Discrete Math. 29(1): 53-76 (1980)

[100] Paul D. Seymour, Robin Thomas: Graph Searching and a Min-Max Theorem for TreeWidth. J. Comb. Theory, Ser. B 58(1): 22-33 (1993)

[101] Paul D. Seymour, Robin Thomas: Call Routing and the Ratcatcher. Combinatorica 14(2): 217-241 (1994)

[102] Kirill Shoikhet, Dan Geiger: A Practical Algorithm for Finding Optimal Triangulations. AAAI 1997: 185-190

[103] Yinglei Song, Chunmei Liu, Russell L. Malmberg, Fangfang Pan, Liming Cai: Tree Decomposition Based Fast Search of RNA Structures Including Pseudoknots in Genomes. CSB 2005: 223-234

[104] Ravi Sundaram, Karan Sher Singh, C. Pandu Rangan: Treewidth of Circular-Arc Graphs. SIAM J. Discrete Math. 7(4): 647-655 (1994)

[105] Jan Arne Telle, Andrzej Proskurowski: Algorithms for Vertex Partitioning Problems on Partial k-Trees. SIAM J. Discrete Math. 10(4): 529-550 (1997) 
[106] Robin Thomas: Tree-Decompositions of Graphs.

http://people.math.gatech. edu/ thomas/SLIDE/CBMS/trdec.pdf

[107] K. Thulasiraman, M.N.S. Swamy: Graphs: Theory and Algorithms. John Wiley \& Sons, Inc., New York, NY (1992)

[108] K. Wagner: Über eine Eigenshaft der ebenen Complexe. Math. Ann. 14: 570-590 (1937)

[109] T.V. Wimer, S.T. Hedetniemi, R. Laskar: A methodology for constructing linear graph algorithms. Congressus Numerantium 50: 43-60 (1985)

[110] Gerhard J. Woeginger: Exact Algorithms for NP-hard Problems: A Survey. Combinatorial Optimization 2001: 185-208

[111] Thomas Wolle: A Framework for Network Reliability Problems on Graphs of Bounded Treewidth. ISAAC 2002: 137-149

[112] Jinbo Xu, Feng Jiao, Bonnie Berger: A Tree-Decomposition Approach to Protein Structure Prediction. CSB 2005: 247-256

[113] Mihalis Yannakakis: Edge-Deletion Problems. SIAM J. Comput. 10(2): 297-309 (1981) 


\section{Nederlandse Samenvatting}

Een boom decompositie van een graaf is een weergave van de graaf door middel van een boom, waarbij de knooppunten van de decompositie deelverzamelingen van de punten van de graaf zijn. De breedte van een boom decompositie wordt gedefinieerd als de omvang van het grootste knooppunt, verminderd met één. De best mogelijke breedte (de kleinste) over alle boom decomposities van een graaf noemen we de boombreedte van de graaf. Een klasse van grafen heeft een begrensde boombreedte als de boombreedte begrensd wordt door een constante die niet afhankelijk is van het aantal punten in de graaf. De boombreedte is een belangrijke structuurparameter van een graaf. Intuïtief geeft ze aan hoe "dik" de boom is waarmee de graaf beschreven kan worden. Het belang van de (begrensde) boombreedte is te vinden in de oplosbaarheid van een groot aantal optimaliseringsproblemen op grafen, zoals minimale kleuring, grootste onafhankelijke verzameling, en diverse netwerkproblemen zoals capaciteitsplanning. Deze problemen kunnen met behulp van de beste boom decompositie, door middel van dynamische programmeringsalgoritmen opgelost worden in een tijd die polynomiaal afhangt van de grootte van de graaf en exponentieel van de boombreedte van de graaf. Met andere woorden: deze problemen kunnen efficiënt opgelost worden op grafen waarvoor de boombreedte begrensd is. Een uitgewerkt voorbeeld van een grafenprobleem dat met behulp van een dynamisch programmeeralgoritme op een boom decompositie wordt opgelost komt in dit proefschrift aan de orde. Helaas is de boombreedte van een graaf niet eenvoudig te bepalen: het probleem is NP-moeilijk. In dit proefschrift wordt een nieuw (exponentieel) algoritme beschreven om de boombreedte exact te bepalen met behulp van een boomzoek methode en een heuristiek voor de boombreedte waarbij met een polynomiaal algoritme goede (maar niet per se optimale) boom decomposities geconstrueerd worden. Daarnaast worden structuren beschreven waarvan bekend is dat ze gerelateerd zijn aan de boombreedte van een graaf, zoals roosters. De aard van de samenhang tussen de boombreedte en deze structuren wordt in dit proefschrift nader onderzocht.

In Hoofdstuk 3 wordt een boomzoek algoritme beschreven voor het exact bepalen van de 
boombreedte. Dit algoritme is gebaseerd op een splitsingsregel die punten paren selecteert en hiervoor twee keuzes laat: het wel of niet samen voorkomen van de twee punten in een knooppunt van de uiteindelijke boom decompositie. Deze regel laat krachtige processing toe, zodat voor kleine grafen (tot ongeveer 30 punten) de boombreedte bepaald kan worden, met name als de graaf weinig kanten heeft in verhouding tot het aantal punten. Voor grotere grafen is dit exacte algoritme in de praktijk echter niet bruikbaar. In Hoofdstuk 4 wordt daarom een heuristische methode beschreven voor het vinden van boom decomposities met kleine, maar niet noodzakelijkerwijs optimale breedte. Deze methode maakt gebruik van een nieuwe buurruimte structuur, waarbij verbonden knooppunten van een decompositie eerst worden samengevoegd en daarna weer worden gescheiden zodanig dat daarmee een nieuwe boom decompositie verkregen wordt. De buurruimte structuur is ingebed in een lokaal zoek algoritme, met meerdere starts. Dit algoritme is competitief met bestaande heuristische methoden voor de boombreedte die onder andere gebaseerd zijn op "tabu search" en "simulated annealing".

In Hoofdstuk 5 wordt gekeken naar planaire grafen. Voor een rooster, een eenvoudig type planaire graaf, geldt dat de boombreedte wordt bepaald door de afmeting van het rooster. Hieruit volgt dat de klasse van planaire grafen geen begrensde boombreedte heeft. Het is nog altijd niet aangetoond dat het bepalen van de boombreedte van planaire grafen een $N P$-moeilijk probleem is. Wel is bekend dat de boombreedte van een planaire graaf van onderen wordt begrensd door zowel de grootte van de grootste rooster minor in de graaf als door de "branchwidth" van de graaf. Ook bestaan er bovengrenzen voor de boombreedte van planaire grafen in termen van de twee bovengenoemde parameters afzonderlijk. In dit proefschrift wordt een klasse van planaire grafen opgesteld waarmee wordt aangetoond dat de bestaande bovengrens voor boombreedte in termen van "branchwidth" scherp is en dat de bovengrens in termen van rooster minoren zeker niet verder aangescherpt kan worden dan tot anderhalf keer de grootte van de grootste rooster minor. Daarbij wordt een nieuwe methode gebruikt om de grootte van de grootste rooster minor te bepalen, gebaseerd op afstanden tussen de vlakken in een planaire inbedding van de graaf. Uit ander onderzoek is inmiddels gebleken dat er een klasse van planaire grafen bestaat waarvoor de boombreedte tweemaal zo groot is als de grootste rooster minor.

Hoofdstuk 6 richt zich tenslotte op de aan- of afwezigheid van subgrafen in een graaf en illustreert het gebruik van boom decomposities in algoritmen. Diverse belangrijke graafklassen kunnen worden gekarakteriseerd door uitsluiting van een verzameling subgrafen. Het grafenprobleem waarvoor in dit hoofdstuk algoritmen worden gepresenteerd luidt: hoeveel kanten dienen minimaal uit een graaf $G$ te worden verwijderd om een verzameling $H$ van grafen niet meer als subgraaf in $G$ aanwezig te laten zijn? Dit probleem is NP-moeilijk voor algemene grafen. Voor speciale gevallen van grafen $G$ met begrensde boombreedte en verzamelingen $H$ presenteren we lineaire constructieve algoritmen, gebaseerd op dyna- 
mische programmering. Verder wordt in dit hoofdstuk aangetoond dat met een laagsgewijze decompositie aanpak een optimale oplossing voor het subgraaf eliminatie probleem op planaire grafen efficiënt kan worden benaderd. 



\section{Curriculum Vitae}

Lambertus Marchal was born on October 20, 1978 in Maarn, The Netherlands. In 1997, he received his Gymnasium diploma from the Van Lodenstein College in Amersfoort. In September of that same year he started studying Applied Mathematics at the University of Twente, where he graduated at the Chair of Discrete Mathematics and Mathematical Programming in January 2005. In September 2005, he started his doctoral research at the department of Quantitative Economics at the Faculty of Economics and Business Administration of Maastricht University, the results of which are presented in this thesis. Since November 2010, Bert has been employed at ABF Research in Delft. 
